\title{
Stability of Methane-Ethane-Propane Mixed Gas Hydrates under Deep Water Conditions
}

\author{
Srinath Chowdary Velaga \\ West Virginia University
}

Follow this and additional works at: https://researchrepository.wvu.edu/etd

\section{Recommended Citation}

Velaga, Srinath Chowdary, "Stability of Methane-Ethane-Propane Mixed Gas Hydrates under Deep Water Conditions" (2014). Graduate Theses, Dissertations, and Problem Reports. 173.

https://researchrepository.wvu.edu/etd/173

This Dissertation is protected by copyright and/or related rights. It has been brought to you by the The Research Repository @ WVU with permission from the rights-holder(s). You are free to use this Dissertation in any way that is permitted by the copyright and related rights legislation that applies to your use. For other uses you must obtain permission from the rights-holder(s) directly, unless additional rights are indicated by a Creative Commons license in the record and/ or on the work itself. This Dissertation has been accepted for inclusion in WVU Graduate Theses, Dissertations, and Problem Reports collection by an authorized administrator of The Research Repository @ WVU.

For more information, please contact researchrepository@mail.wvu.edu. 


\title{
Stability of Methane-Ethane-Propane Mixed Gas Hydrates under Deep Water Conditions
}

\author{
Srinath Chowdary Velaga
}

Dissertation submitted

to the Benjamin M. Statler College of Engineering and Mineral Resources at West Virginia University

in partial fulfillment of the requirements for the degree of

Doctor of Philosophy

in

Chemical Engineering

Brian Anderson, PhD., Chair

Richard Turton, PhD. Charter Stinespring, PhD. Ismail Celik, PhD. Brian Popp, PhD.

Department of Chemical Engineering

Morgantown, West Virginia

2014

Keywords: Mixed gas hydrates, $\mathrm{H}-\mathrm{L}_{\mathrm{w}}$ equilibrium, molecular dynamics simulations Copyright 2014 Srinath Chowdary Velaga 


\title{
ABSTRACT \\ Stability of Methane-Ethane-Propane Mixed Gas Hydrates under Deep Water Conditions
}

\author{
Srinath Chowdary Velaga
}

Methane hydrates are globally distributed in sediments along the continental margins and potentially contain more energy than all fossil fuel reserves. However, methane is also a potential greenhouse gas which could play a major role in global climate change. Understanding the stability of gas hydrates can help us to understand their role in the climate change. Three main factors affect the stability of hydrates: Temperature $(T)$, Pressure $(P)$ and composition. Hydrates become unstable when they are exposed to pressures and temperatures outside the hydrate stability zone (HSZ) in a process commonly called dissociation. However, hydrates can also become unstable even when the pressure and temperature are within the HSZ but the concentrations of the hydrate forming gases are below their fully-saturated levels in the surrounding water phase. This process can be described as dissolution. In situ observations of marine outcrops of gas hydrates indicate that these hydrates exposed to surrounding seawater are more stable than predicted using diffusion-controlled models based on the surrounding methane saturations.

Naturally-occurring hydrates may not be simple structure I (sI) methane hydrates and may contain higher hydrocarbons like ethane, propane etc., which forms structure II (sII) hydrates. Therefore, these mixed hydrates may act to stabilize the hydrates. In this work, the dissolution of sII hydrates in the presence of water has been studied using molecular dynamics (MD) simulations to understand if and how the presence of ethane and propane may stabilize the hydrate. Lattice constants for sII hydrates were calculated and compared to experimental values to validate the OPLS potentials used for the hydrocarbon guest molecules. The effect of higher hydrocarbons, such as ethane and propane, on the stability of gas hydrate was studied by changing the composition in the hydrate phase keeping the methane composition constant in the large cage and small cages. Also, the effect of methane composition was also studied by changing the methane occupancy in large and small cages. MD simulations reveal that the fully occupied hydrate is more stable than the presence of empty cages. The number of methane molecules moved into the liquid phase from the hydrate phase has been increased with the decrease in the large cage occupancy. No effect was found on the dissolution of sII hydrate by changing the small cage occupancy from $100 \%$ to $81.5 \%$. The dissolution of sII hydrate was linear in the first few nanometers of the simulations and then observed an oscillatory behavior; this oscillatory behavior is due to the hydrate formation and dissociation at the hydrate-water layer interface.

The probable phase in the marine sediments can be a two phase hydrate $(\mathrm{H})$-Liquid water $\left(\mathrm{L}_{\mathrm{w}}\right)$ thermodynamic equilibrium in the absence of vapor phase. Understanding the fate and transport of hydrocarbons and hydrocarbon mixtures in the deepsea and underlying sediments requires accurate determination of this two phase $\mathrm{H}-\mathrm{L}_{\mathrm{w}}$ thermodynamic equilibrium in the absence of a free gas phase. In addition to controlling hydrate formation directly from the aqueous phase, the $\mathrm{H}-\mathrm{L}_{\mathrm{w}}$ equilibrium also provides the aqueous solubility of dissolving hydrate. The two phase $\mathrm{H}-$ $\mathrm{L}_{\mathrm{w}}$ thermodynamic model is based on the van der Waals and Platteeuw model and the Holder 
model. The Langmuir constants, an important term, in the van der Waals Platteeuw model were calculated from cell potential parameters obtained from $a b$ initio intermolecular potentials and the experimental data, i.e. it does not rely on empirical fitting parameters. Variable reference parameters were used for each guest molecule instead of using single value for all the guests. The Pitzer model was used to calculate the activity of seawater. The solubilities of pure methane, ethane, and propane in water at $\mathrm{H}-\mathrm{L}_{\mathrm{w}}$ equilibrium are compared to the available experimental data and shown to be as accurate as the experimental data. The model predictions show that the ratio of large to small cage occupancy decreasing with increase in temperature or pressure. The prediction of the model shows that at the two phase $\mathrm{H}-\mathrm{L}_{\mathrm{w}}$ equilibrium in the presence of electrolytes forms the hydrate at lower pressures compares to the pure water at a given temperature and dissolved hydrocarbon solubility. Thus the presence of electrolyte promotes the hydrate formation rather than acting as an inhibitor. This is in reverse to that in the three phase vapor-liquidwater-hydrate $\left(\mathrm{VL}_{\mathrm{w}} \mathrm{H}\right)$ region where it is well known that salts act as an inhibitor to hydrate formation. Finally, the methane-ethane-propane ratio from the Macondo oil spill had been used as a typical thermogenic hydrocarbon mixture and hydrocarbon solubilities at $\mathrm{H}-\mathrm{L}_{\mathrm{w}}$ equilibrium under deepsea conditions have been presented for pure water and seawater. 


\section{Acknowledgments}

I would like to acknowledge the following people and organizations that helped me in various ways throughout the duration of this project.

Firstly, I am very grateful to my advisor, Professor Brian Anderson for his support, skilful guidance and encouragement throughout my doctoral studies to accomplish this challenging research project. He has been more than an academic advisor to an international student like me. I have learned many things from him, how to interact with people in an appropriate way, analyze the problem. I wish I knew a better word than "thank you"

I would also like to thank National Energy Technology Laboratory (NETL) of U.S. Department of Energy (DOE) for providing the funding to this project. I would also like to thank Dr. Jonathan Levine and Robert Warzinski from NETL, Pittsburgh for their help in completing my dissertation.

I also would like to thank the present and past members of Anderson lab: Dr. Manohar Gaddipati, Dr. Nagasree Garapati, Xiaoning He, Madhur Bedre, Terry Ryan, Dr. Patrick McGuire, Vikas Agarwal, Prathyusha Sridhara, Taiwo Ajayi, Manish Nandanwar. A special thanks to Dr. Manohar Gaddipati and Dr. Nagasree Garapati for all the discussion and fun I had with them while working in the lab.

I would like to thank my parents and my brother for their inseperable support and prayers and their love, affection and encouragement in all the phases of my life. Without your unending support and love from childhood to now, I would never made it throught this process or any of the tough time in my life

Finally, I would also like to thank my roommate, Sridhar Chouhan, for all the help, support, love and encouragement for the past 6 years. Also, would like to thank all my friends in 
Morgantown in no particular order they are: Pratik, Saurabh, Manasa, Akshaya, Rutika, Kiran, Pallavi Sahiti, Deeban and all who directly or indirectly helped me in completing my work. Thank you very much 
Dedicated to my parents

and

To my Brother 


\section{Contents}

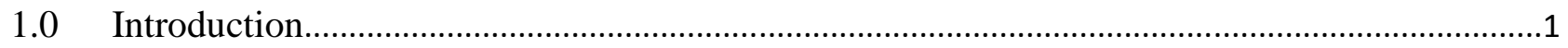

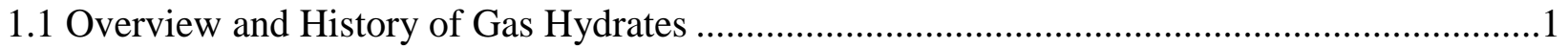

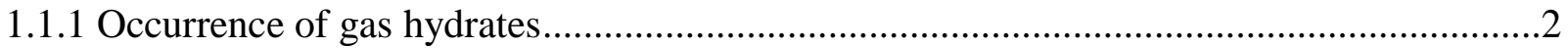

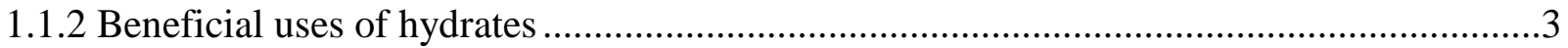

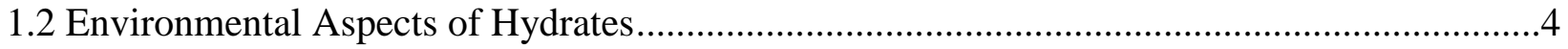

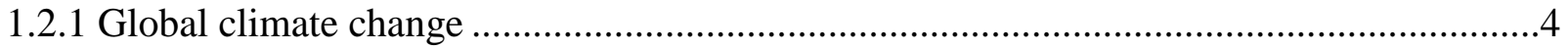

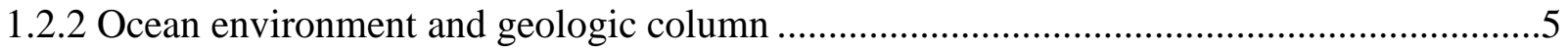

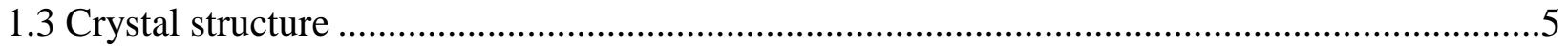

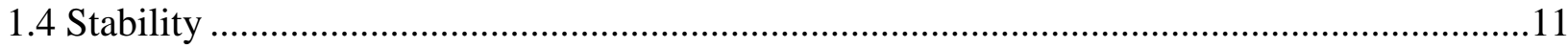

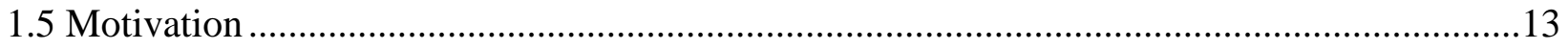

1.5.1 Dissolution.............................................................................................................

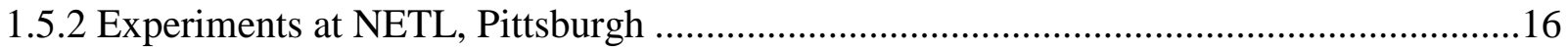

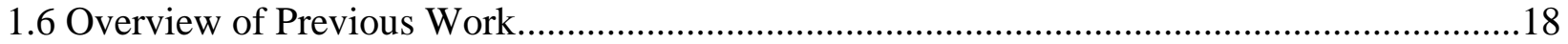

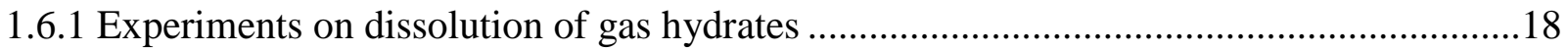

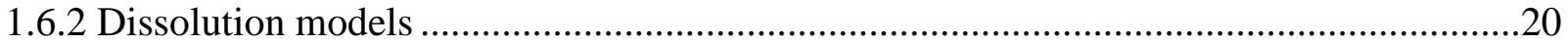

1.6.3 Dissolution and dissociation studies using MD simulations ..........................................22

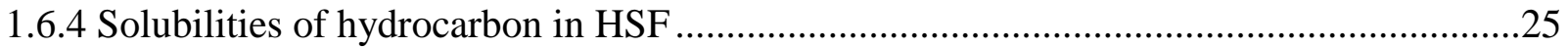

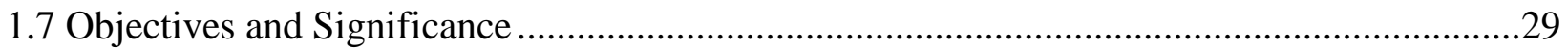

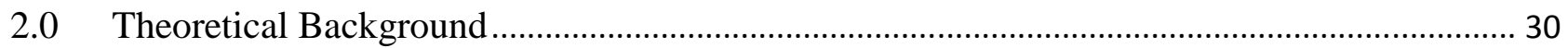

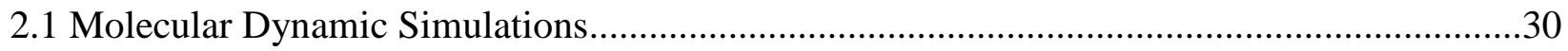

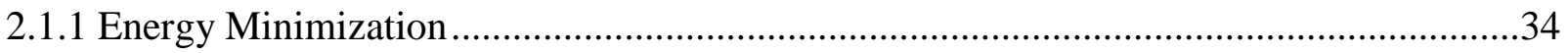

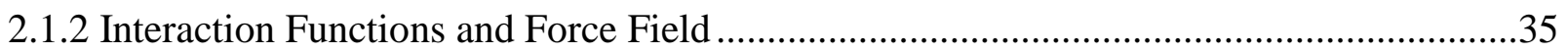

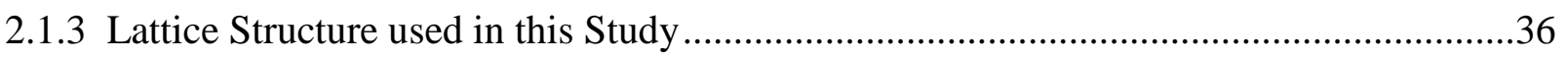

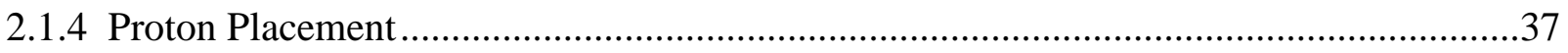

2.2 Thermodynamic Model .............................................................................................

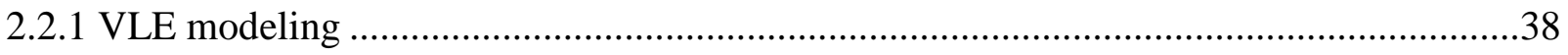

2.2.2 Gas hydrate phase equilibrium ....................................................................................

3.0 Molecular Dynamics Simulations to Study the Dissolution of Structure II Hydrates

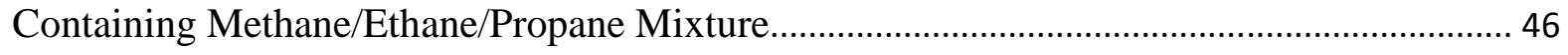

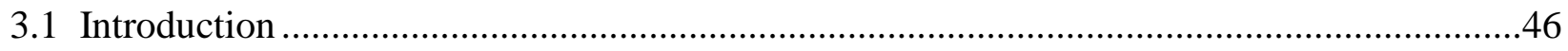

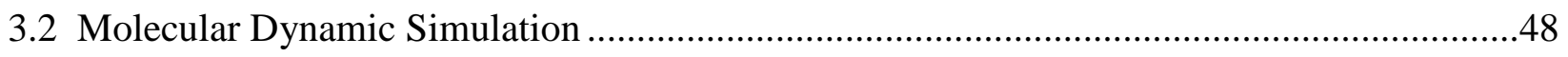

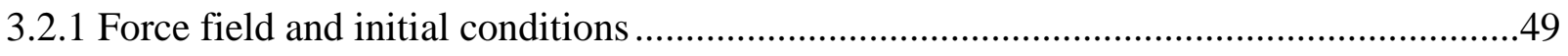

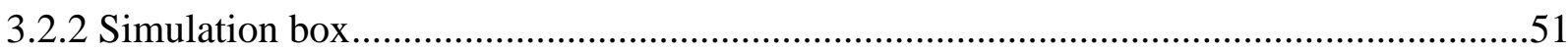




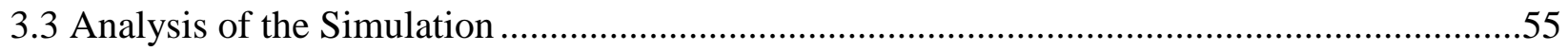

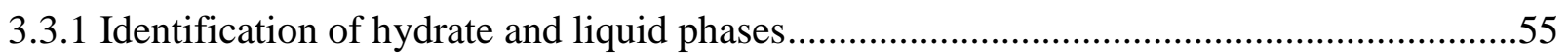

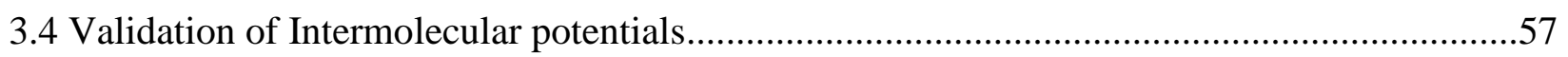

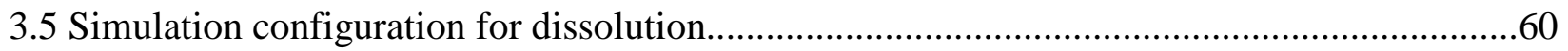

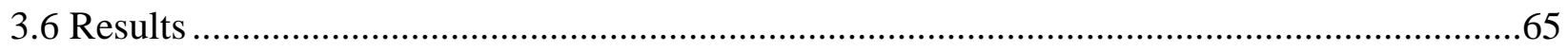

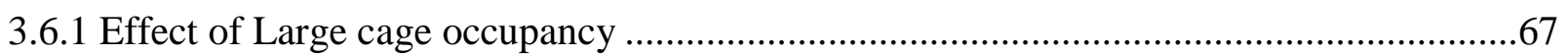

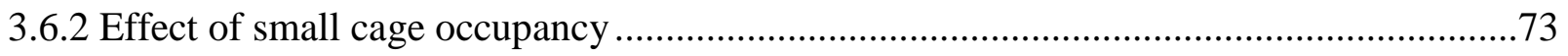

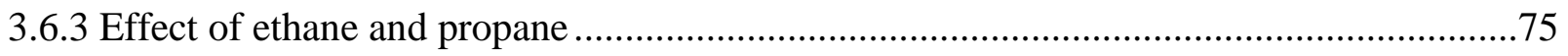

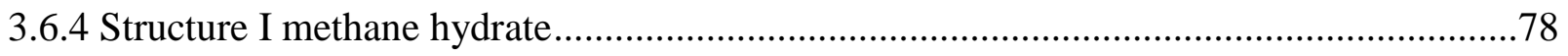

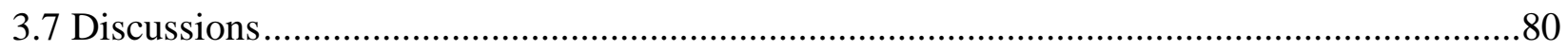

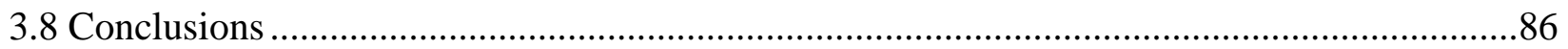

4.0 A Thermodynamic Model to Predict the Hydrocarbon Solubility in Water at Two Phase

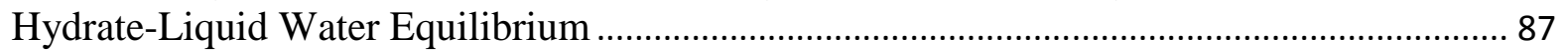

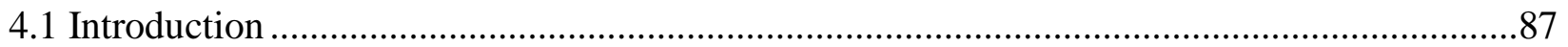

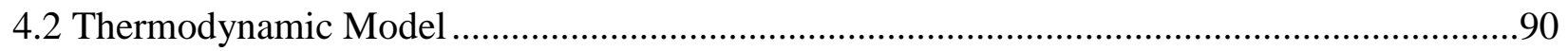

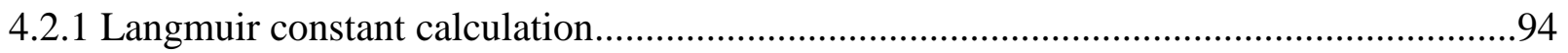

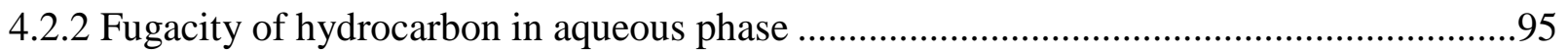

4.2.3 Activity of water and activity coefficients in seawater .................................................97

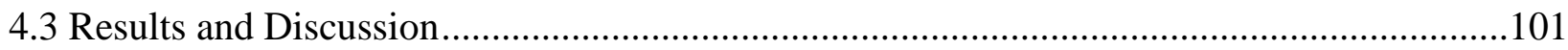

4.3.1 H- $\mathrm{L}_{\mathrm{w}}$ Equilibrium for Pure Components ....................................................................

4.3.2 H-L $\mathrm{L}_{\mathrm{w}}$ Equilibrium for Mixed Components ..................................................................108

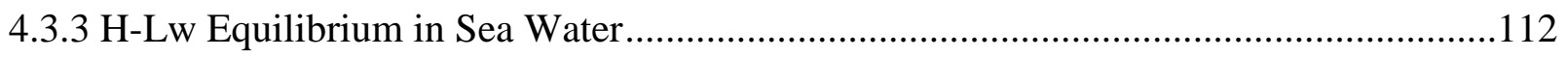

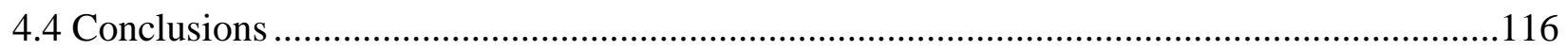

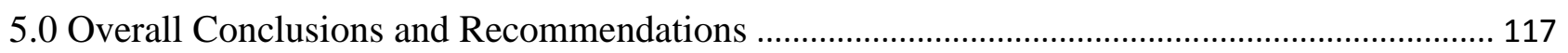

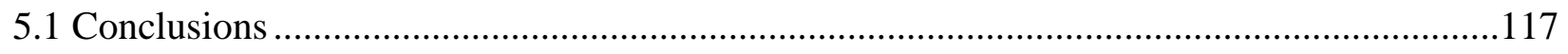

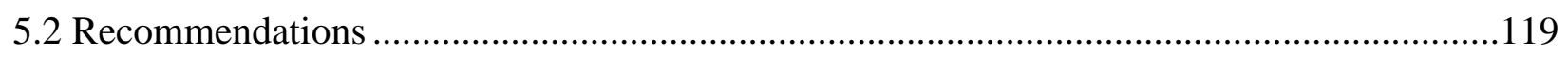

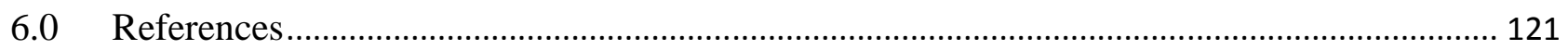

Appendix A: The oxygen and hydrogen positions of water in sII hydrate unit cell......................... 138 


\section{List of Tables}

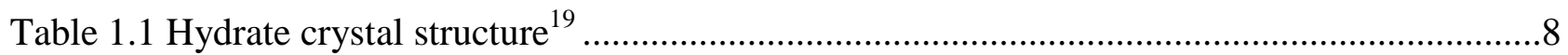

Table 2.1 Heat capacity and volumetric reference properties between the empty hydrate lattice and fluid phase (liquid water or ice) ............................................................................43

Table 3.1. Intermolecular potential parameters for methane, ethane, propane and water. Atoms marked in bold indicate interacting site. ...........................................................50

Table 3.2 Relative positions of all cavities in a unit cell of sII hydrate showing cavity centers.......52

Table 3.3 Cartesian coordinates of methane molecule with respect to the central atom carbon ........53

Table 3.4 Cartesian coordinates of ethane molecule. The midpoint between the $\mathrm{C} 1$ and $\mathrm{C} 2$ is the

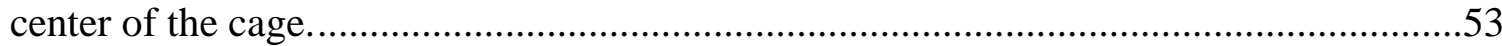

Table 3.5 Cartesian coordinates of propane molecule. The center of the cage is at atom $2 \mathrm{C}$..........54

Table 3.6 Cage occupancies in the sII hydrate and number of guest gas molecules in the simulations

Table 3.7 Effect of cage occupancy on the dissolution of sII hydrate ........................................77

Table 4.1 The values of reference properties for structure I and structure II hydrates....................93

Table 4.2 Cell potential parameters for structure I hydrate ....................................................95

Table 4.3 Cell potential parameters for structure II hydrates .................................................95

Table 4.4 Partial molar volume of hydrocarbons...................................................................97

Table 4.5 The temperature dependence of the Debye-huckel parameters and for the binary interaction parameters of Pitzer model ${ }^{147}$ determined by Spencer et al. (1990) ${ }^{148}$...........99

Table 4.6 The temperature dependence of mixed electrolyte parameters of Pitzer model ${ }^{147}$ determined by Spencer et al. (1990) ${ }^{148}$

Table 4.7 Salting coefficients for methane ${ }^{150}$, ethane ${ }^{150}$ and propane in electrolyte solution at $25^{\circ} \mathrm{C}$

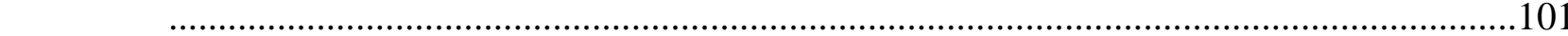

Table 4.8 Experimental methane solubility data at $\mathrm{H}-\mathrm{Lw}$ equilibrium ..................................... 103

Table 4.9 Occupancy ratio, $(\boldsymbol{\theta} \boldsymbol{L} \boldsymbol{\theta S})$, of methane sI hydrates at $\mathrm{H}-\mathrm{L}_{\mathrm{w}}$ equilibrium ......................104

Table 4.10 Solubility of ethane in water at $\mathrm{H}-\mathrm{L}_{\mathrm{w}}$ equilibrium.................................................107

Table 4.11 Solubility of Propane in water at $\mathrm{H}-\mathrm{L}_{\mathrm{w}}$ equilibrium ..............................................107

Table 4.12 Molality $\left(\mathrm{mol} / \mathrm{kg} \mathrm{H}_{2} \mathrm{O}\right)$ of ions in $35 \mathrm{wt} \%$ sea water concentration ...........................112 


\section{List of Figures}

Figure 1.1 Cavities of hydrate structure: (a) pentagonal dodecahedron $\left(5^{12}\right)$, (b) Terakaidecahedron $\left(5^{12} 6^{2}\right)$, (c) Hexakaidecahedran $\left(5^{12} 6^{4}\right)$.

Figure 1.2 Cavities of hydrate structures (a) Irregular dodecahedron $\left(4^{3} 5^{6} 6^{3}\right)$, (b) Icosahedron $\left(5^{12} 6^{8}\right)$ .8

Figure 1.3 Structure I hydrate in 2 adjacent unit cells in $\mathrm{x}$-direction ......................................10

Figure 1.4 Structure II hydrate in one unit cell of cubic structure .............................................10

Figure 1.5 Structure $H$ hydrate in 2 adjacent unit cells of hexagonal structure in $\mathrm{x}$-direction...........11

Figure 1.6 Phase diagram for methane hydrate showing hydrate stability zone (HSZ) in the ocean settings. Hydrates are formed in the HSZ, anything above or below destabilizes the

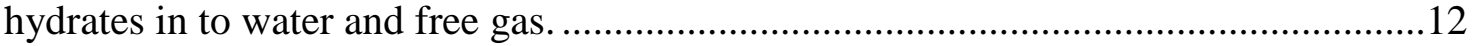

Figure 1.7 Schematic of the high pressure water tunnel facility (HWTF) at NETL, Pittsburgh. The high pressure water tunnel (HWT) is shown on the left side. Detail description about the apparatus can be found at Warzinski et al. $(2008)^{37}$

Figure 2.1 Periodic boundary conditions ${ }^{87}$. This figure is taken from Allen, Comp. S. Matter, NIC

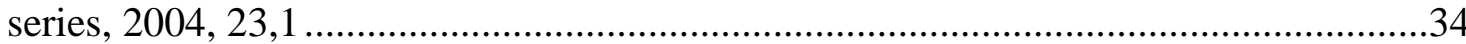

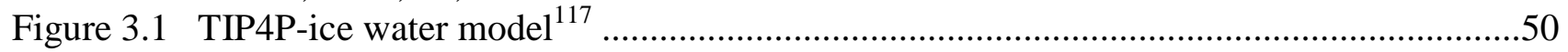

Figure 3.2 Ball and stick model of (a) Methane, (b) Ethane and (c) Propane molecules.................54

Figure 3.3 A 2-dimensional view of an 8 unit cell $(2 \times 2 \times 2)$ simulation box sII hydrate with methaneethane-propane guest molecules. Methane is shown in cyan; ethane in green; propane

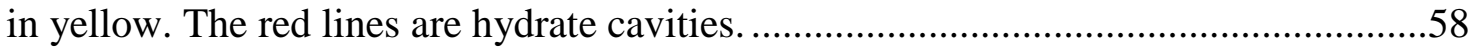

Figure 3.4 Equilibriation of lattice constant for $2 \times 2 \times 2$ sII hydrate unit cells at $260 \mathrm{~K}$.................59

Figure 3.5 Effect of temperature on the lattice constant of sII hydrate. The experimental value at $275 \mathrm{~K}$ is $1.738 \mathrm{~nm}$ and the value obtained by simulation is $1.732 \mathrm{~nm}$.......................59

Figure 3.6 Snap shot of the initial simulation box showing the hydrate-water interface. Hydrate is shown in red color lines (hydrogen bonded lines). Liquid water layer: water of oxygen is in red color and hydrogen in white .62

Figure 3.7 Density plot of guest gas molecules across the simulation box to view the change in number density of the gas molecules at $0 \mathrm{~ns}$ and at $30 \mathrm{~ns}$ simulations for (a) methane, (b) ethane (c) propane .66

Figure 3.8 Snapshot of the sII mixed hydrate-water system simulation box containing methane, ethane and propane guest molecules for Model 1. Methane molecules are colored in cyan, ethane molecules are colored in orange, propane molecules are colored in yellow. Water molecules are shown in red lines as hydrogen bonds... 67

Figure 3.9 The amount of methane molecules moved in to the water phase from sII hydrate phase at different large cage occupancies (Model 1 to Model 5).

Figure 3.10 Snapshot of the simulation box for Model 5 at the end of simulation. Methane molecules are colored in cyan, ethane molecules are colored in orange, propane molecules are colored in yellow. Water molecules are shown in red lines as hydrogen bonds............70

Figure 3.11 Number density plot of the guest molecules in the hydrate phase along the z-direction of the box. (a) methane, (b) ethane (c) propane. 71

Figure 3.12 Number of $\mathrm{CH}_{4}$ molecules moved in to the bulk liquid water from hydrate phase during the course of the simulation at different large cage occupancy (L). The small cage is 
100\% occupied. Model 1-100\%, Model 2-88\%, Model 3-81\%, Model 4-63\%, Model 5-50\% large cage occupancy ................................................................................

Figure 3.13 Effect of small cage occupancy (S) on the dissolution of methane molecules to the liquid water phase from the hydrate phase for 100\% small cage occupancy (Model 1, Model 2 and Model 5) and 81.25\% small cage occupancy (Model 6, Model 7 and Model 8)...74

Figure 3.14 Number of $\mathrm{CH}_{4}$ molecules that moved in to the bulk liquid water from hydrate phase during the course of the simulation at $81.25 \%$ small cage occupancy and at different large cage occupancy $(\mathrm{L})$.......................................................................................74

Figure 3.15 Number of $\mathrm{CH}_{4}$ molecules that moved in to the bulk liquid water from hydrate phase during the course of the simulation at $81 \%$ large cage occupancy. L-denotes for large cage occupancy.....

Figure 3.16 Number of $\mathrm{CH}_{4}$ molecules that moved in to the bulk liquid water from hydrate phase during the course of the simulation at $76 \%$ large cage occupancy. L-denotes for large cage occupancy.... .76

Figure 3.17 Number of methane molecules dissolved in to the liquid water for sI methane hydrate. 78

Figure 3.18 Number of methane molecules moved in to the liquid water during the course of the simulation at different percentage of cage occupancies for sI methane hydrate............79

Figure 3.19 Dissolution of methane molecules from hydrate phase to the bulk liquid water during the course of the simulation. The lines are for total cage occupancy of $87.5 \%$ obtained by emptying small cage and by emptying large cage. 82

Figure 3.20 Dissolution of methane molecules into the liquid water as a function of (a) Propane large cage occupancy (b) Ethane large cage occupancy. ...................................................... 84

Figure 3.21The number of water molecules in the hydrate phase during the course of the simulations. The small cage is $100 \%$ occupied. L-denotes large cages ..........................85

Figure 4.1 Comparison of mole fraction of methane in water at $\mathrm{H}-\mathrm{L}_{\mathrm{w}}$ equilibrium predicted by this model (dotted lines) and by the expression given by Lu et al. ${ }^{68}$ (symbols). The bottom solid line is the VLH equilibrium for methane hydrate, plotted from the data obtained from Sloan ${ }^{134}$ .103

Figure 4.2 Cage occupancy ratio $(\boldsymbol{\theta} \boldsymbol{L} \boldsymbol{\theta} \boldsymbol{S})$ of sI methane hydrate at $\mathrm{H}-\mathrm{L}_{\mathrm{w}}$ equilibrium predicted by this model. 105

Figure 4.3 Cage occupancy of sI methane hydrate at $\mathrm{H}-\mathrm{L}_{\mathrm{w}}$ equilibrium predicted by this model..105 Figure 4.4 Dissolved hydrocarbon mixture concentration in the aqueous phase in equilibrium with gas hydrates at different temperatures and pressures. The vertical dotted lines are for total hydrocarbon mole fraction $(x \boldsymbol{C 1 C 2 C 3})$ in aqueous phase with composition $\mathrm{C}_{1}=$ $87.5 \%, \mathrm{C}_{2}=8.1 \%$ and $\mathrm{C}_{3}=4.4 \%$. Also shown is the $\mathrm{VL}_{\mathrm{w}} \mathrm{H}$ equilibrium curve with solid black line.

Figure 4.5 Gas hydrates cage occupancies at two phase equilibrium $\left(\mathrm{L}_{\mathrm{w}}-\mathrm{H}\right)$. The symbols are for different total hydrocarbon solubility in water with composition, $\mathrm{C}_{1}=87.5 \%, \mathrm{C}_{2}=$ $8.1 \%$ and $\mathrm{C}_{3}=4.4 \%$ when in equilibrium with hydrate phase.................................110

Figure 4.6 Hydrate phase composition at two phase equilibrium $\left(\mathrm{L}_{\mathrm{w}}-\mathrm{H}\right)$. The symbols are for different total hydrocarbon solubility in water with composition, $\mathrm{C}_{1}=87.5 \%, \mathrm{C}_{2}=$ $8.1 \%$ and $\mathrm{C}_{3}=4.4 \%$ when in equilibrium with hydrate phase. 111

Figure 4.7 Solubility of methane in seawater at two phase hydrate -liquid seawater equilibrium. The vertical lines are aqueous methane mole fraction 113

Figure 4.8 Effect of salinity in the formation of methane hydrate from the dissolved methane concentrations. The dotted lines are for seawater and solid lines are for pure water. The 
$\mathrm{VL}_{\mathrm{w}} \mathrm{H}$ equilibrium curve with solid black line and dotted line is for pure water and seawater respectively.

Figure 4.9 Dissolved hydrocarbon mixture concentration in the aqueous phase in equilibrium with gas hydrates at different temperatures and pressures for seawater and fresh water. The vertical dotted lines for seawater and solid lines for fresh water. Each vertical line is for total hydrocarbon mole fraction $(\boldsymbol{x C 1 C 2 C 3})$ in aqueous phase with composition $\mathrm{C}_{1}=$ $87.5 \%, \mathrm{C}_{2}=8.1 \%$ and $\mathrm{C}_{3}=4.4 \%$. Also shown is the $\mathrm{VL}_{\mathrm{w}} \mathrm{H}$ equilibrium curve for fresh water and seawater with solid black and dotted black line respectively...................115 


\subsection{Introduction}

\subsection{Overview and History of Gas Hydrates}

Gas hydrates, also known as gas clathrates, are class of solids in which low molecular weight gas molecules $\left(\underline{\mathrm{O}}_{2}, \underline{\mathrm{H}}_{2}, \underline{\mathrm{N}}_{2}, \underline{\mathrm{CO}}_{2}, \underline{\mathrm{CH}}_{4}, \underline{\mathrm{H}}_{2} \underline{\mathrm{S}}, \underline{\mathrm{Ar}}, \underline{\mathrm{Kr}}\right.$, and $\left.\underline{\mathrm{Xe}}\right)$ occupy cages made of hydrogen-bonded water molecules. The presence of the guest molecule thermodynamically stabilizes the structure. The term clathrate was first used by Powell ${ }^{1}$ after the Latin word clathrates meaning to be enclosed or protected by cross bars of a grating. In 1811, Sir Humphrey Davy ${ }^{2}$ discovered the first gas hydrates; he observed a yellow precipitate while passing chlorine gas through water at temperature near $0^{\circ} \mathrm{C}$ and identified the solid as chlorine hydrate. In addition, there was some evidence that hydrates were retrieved prior to Davy by Joseph Priestley ${ }^{3}$ in 1778. Priestley observed that the vitriolic air $\left(\mathrm{SO}_{2}\right)$ would impregnate water and cause it to freeze and refreeze to form $\mathrm{SO}_{2}$ hydrate. Wroblewski ${ }^{4-5}$ might be the first to record the evidence of the existence of $\mathrm{CO}_{2}$ hydrate during his studies on carbonic acid. He observed a white material resembling snow, gas hydrate, formed by raising the pressure above certain limit in his $\mathrm{CO}_{2}-\mathrm{H}_{2} \mathrm{O}$ system.

During the first hundred years after Davy's discovery of gas hydrates, the studies on gas hydrates were academic, concerned with the identification of species that form hydrates and the pressure-temperature conditions at which this formation occurs. In 1934, Hammerschmidt ${ }^{6}$ indicated that the plugging of natural gas pipeline was not due to the formation of ice but due to the formation of clathrate hydrates of natural gas. Considering the significant economic risks in the gas and oil industry, and that the industry was growing rapidly, a great deal of research has been conducted by the petroleum industry in order to inhibit this phenomenon. It marked the beginning of the intense research on natural gas hydrates by the oil and gas industry, government and 
academia. Since the mid 1960's, with the discovery of natural gas hydrates, hydrate research has been motivated by production, transport and processing problems in unusual environments such as the North Slope of Alaska, in Siberia and in deep ocean drilling.

\subsubsection{Occurrence of gas hydrates}

Naturally occuring gas hydrates can be found on the seafloor, in ocean sediments, in deep lake sediments, as well as in the permafrost regions. Huge deposits of carbon $\left(2 \times 10^{16} \mathrm{~kg}\right)$ are trapped in oceanic sediments in the form of methane hydrates. Natural deposits of methane gas hydrates were first discovered in the Soviet Union in the early 1960's and later in many marine types of sediment and in Alaskan permafrost ${ }^{7}$. These hydrates represent a potential energy source that could possibly last for thousands of years. However, the estimate of the amount of hydrates decreases as man learns more about hydrates in the environment. The initial global hydrate reserve estimation was given by Trofimuk ${ }^{8}$ with an estimate of $3053 \times 10^{15} \mathrm{~m}^{3}$ of methane assuming hydrates could occur wherever sufficiently low temperatures and high pressures exist. Soloview ${ }^{9}$ considered the limiting factors like availability of methane, limited porosity, percentages of organic matter and so on in estimating the hydrate reserve and gave the minimum of all the researches with an estimate of $0.2 \times 10^{15} \mathrm{~m}^{3}$ methane. Klauda and Sandler ${ }^{10}$ presented an equilibrium thermodynamic model for in-place hydrate formation, a different method of estimating hydrates reserves from those of all preceding estimates. They generated a new ab initio thermodynamic model which includes the effect of water salinity, confinement of hydrate in pores and the distribution of pores in the natural sediments to predict the hydrate stability in the sea floor. Using this model and a mass transfer description of hydrate formation they predicted the occurrences of methane hydrates. They estimated a total volume of $120 \times 10^{15} \mathrm{~m}^{3}$ of methane gas but this estimates includes very deep hydrates and dispersed small concentrations of hydrates that may dissociates 
during recovery. When only continental margins are considered they estimated $44 \times 10^{15} \mathrm{~m}^{3}$ of methane gas expanded to standard temperature and pressure. The energy consumption of the United States for 1000 years at current rate is $1 \times 10^{15} \mathrm{~m}^{3}$. Therefore, the resource of hydrates has a potential of providing the clean energy source for up to 10,000 years ${ }^{11}$. Destabilized methane hydrates may have some effect on the global climate change, methane has green house gas properties, but this effect will probably be minimal at least during the next 100 years ${ }^{12}$.

\subsubsection{Beneficial uses of hydrates}

Hydrates have also been considered as a possible solution to the $\mathrm{CO}_{2}$ problem. The idea of sequestrating the carbon dioxide on the ocean floor to hold the increase in green house gas in the atmosphere has been proposed. Liquid $\mathrm{CO}_{2}$ is injected in to the deep regions of the ocean at depths greater than $1000 \mathrm{~m}$ to form solid clathrates. It is also proposed that the $\mathrm{CO}_{2}$ can be stored in linkage with methane exploitation, as the hydrate formation and dissociation conditions of $\mathrm{CO}_{2}$ and methane hydrates are different. This swapping process will help in the sequestering the $\mathrm{CO}_{2}$ and also the source for methane. A microscopic analysis was conducted by Park et al. ${ }^{13}$ to examine the swapping of $\mathrm{CO}_{2}$ and methane hydrate: for structure $\mathrm{I} \mathrm{CH}_{4}$ hydrate, the $\mathrm{CO}_{2}$ molecules preferably occupy the large cages recovering $64 \%$ of the methane and for structure $\mathrm{II} \mathrm{CH}_{4}$ hydrate (mixed hydrate with ethane), a structural transition from structure II to structure I and a lattice dimension change occurs. They showed that the recovery of methane gas increased to $84 \%$ when nitrogen is added with $\mathrm{CO}_{2}$ gas. Gas hydrates have been proposed and used in a number of separation processes. They have been used successfully in the desalination of seawater ${ }^{14}$ and in the separation of light gases. Hydrates also have the potential to separate the $\mathrm{CO}_{2}$ gas from the flue gases exhausted by the large power plants ${ }^{15}$. The transportation and storage of natural gas in the form of solid gas hydrates has also been suggested ${ }^{16}$, because of the ability of hydrates to store higher 
concentrations $^{17}$. Hydrate storage of gases has benefits of lower storage space and low pressures for safety. Finally, the use of their dissociation energy can be applied in a refrigeration process or cool storage.

\subsection{Environmental Aspects of Hydrates}

The study of impacts of gas hydrates on the earth's climate has been a challenge, especially related to methane evolution and $\mathrm{CO}_{2}$ sequestration. The amount of methane present in gas hydrate onshore and offshore was said to be around 3000 times the amount in the present atmosphere. Destabilization of oceanic hydrates can release large amounts of methane in to the ocean and atmosphere. Methane being a green house gas can be major cause of the global warming. So, the stability of seafloor gas hydrates and a continuous change in the global climate could be interrelated.(Kennett et al.2002).

\subsubsection{Global climate change}

Global warming, a steady increase in the global temperature of the earth's atmosphere, has been a problem over the last century because it has been occurring at a rapid rate. The increase in the greenhouse gases in the atmosphere has been considered as one of the major causes of global warming. Since 1850, the amounts of three main greenhouse gases $\mathrm{CO}_{2}$, methane and nitrous oxide in the atmosphere have been increasing at extraordinary rapid rates. The amount of increase in the levels of methane has been $142 \%$ from 1750 to 1990 while the current level is around $1.774 \mathrm{ppm}$ in $2005^{14}$. It is predicted that most of these increased greenhouse gas emissions are due to the human activities such as the extensive use of fossil fuels. However, effects of gas hydrates on the global climate have been an area of research for a long time. The $\mathrm{CO}_{2}$ concentration levels in the atmosphere are much higher compared to methane, but its greenhouse effects are lesser compared to methane. Methane is an eight fold stronger greenhouse gas than carbon dioxide on a molecule- 
for-molecule basis ${ }^{15}$. Methane is a transient species in the atmosphere because it can oxidize itself to $\mathrm{CO}_{2}$. So, it may have a lifetime of about a decade in the atmosphere, unlike $\mathrm{CO}_{2}$, which when accumulated in the atmosphere persists to affect climate for hundreds of years.

\subsubsection{Ocean environment and geologic column}

Gas hydrates in many cases are stable near sea floor conditions ${ }^{18}$. Destabilization of seafloor gas hydrates affects the ocean environment and geologic column along with the atmosphere. Methane, when released from the seafloor sediments as a consequence of destabilization of gas hydrates would be present in the water column in the ocean before escaping in to the atmosphere. A part of the methane released in to the water column would dissolve and get microbially oxidized to $\mathrm{CO}_{2}$. The ocean has the capacity to absorb vast amounts of carbon dioxide, but it would make the ocean water more acidic. This could make life difficult for marine organisms that build shells and skeletons out of calcium carbonate. The ecological effects of a near $3^{\circ} \mathrm{C}$ increase are estimated to result in large scale degradation of coral reeves, which are a vital habitat for many ocean species.

\subsection{Crystal structure}

Hydrates are formed due to the unusual behavior of the $\mathrm{H}_{2} \mathrm{O}$ molecules. Hydrates, as well as ice are formed due to the ability of water to form hydrogen bonds. Each water molecule is attached to four neighboring water molecules through hydrogen bonding. The oxygen atoms of the $\mathrm{H}_{2} \mathrm{O}$ molecules are tetrahedrally coordinated in the clathrates hydrate but not as regularly as in the ice. This deviation from regularity is due to the polyhedra (a combination of hexagonal, pentagonal and square faces) formed from hydrogen bonded water molecules. The combination of these basic

cavities forms different hydrate structures ${ }^{19}$. Clathrate hydrate can possess many different crystal 
structures ${ }^{18}$, but only three structures are known to occur in natural environments: structure I (sI), structure II (sII) and structure $\mathrm{H}(\mathrm{sH})$. The nomenclature suggested by Jeffry and McMullan ${ }^{19}$ for basic cavities of hydrate structures is $n^{m}$, where $n$ is the number of edges and $m$ is the number of faces. Hydrate structures are composed of five basic polyhedral cavities formed by hydrogenbonded water molecules. The pentagonal dodecahedron $\left(5^{12}\right)$ has 12 pentagonal faces with equal edge lengths and angles. The tetrakaidecahedron $\left(5^{12} 6^{2}\right)$ has 12 pentagonal and 2 hexagonal faces. Three other polyhedral cavities are the hexakaidecahedran $\left(5^{12} 6^{4}\right)$, the irregular dodecahedron $\left(4^{3} 5^{6} 6^{3}\right)$, and the icosahedron $\left(5^{12} 6^{8}\right)$. Since the cavities are expanded relative to ice, hydrate cavities are stabilized by the repulsive presence of guest molecules in the cavity. The size of the guest gas molecule and the properties of the guest gas determine the structure of the gas hydrate ${ }^{19}$. Structure I and II hydrates are known to occur commonly in nature while structure $\mathrm{H}$ hydrate is a rare kind of hydrate known to exist where there is a source of thermogenically produced gases containing hydrocarbons ${ }^{20}$. Structure I and II gas hydrates are usually formed by gas molecules of smaller size and can be stabilized by filling $70 \%$ of the cavities by a single guest molecule, unlike structure $\mathrm{H}$ which requires the cooperation of two guest gas molecules, one large and one small to be stable. Different cavities which combine to form the three different structures are shown in Figure 1.1 and Figure 1.2. The specifications of each type of cavity in all the three structures are given in Table 1.1. 


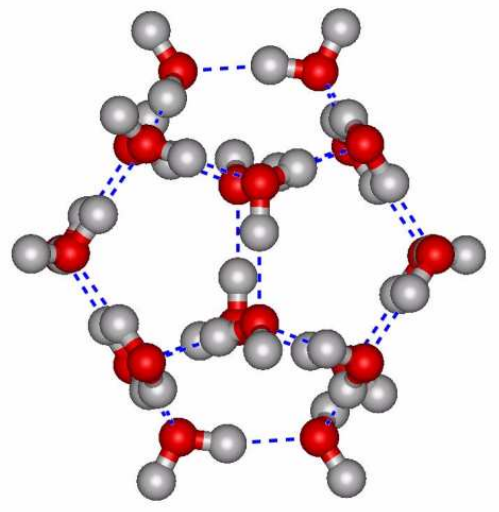

(a)

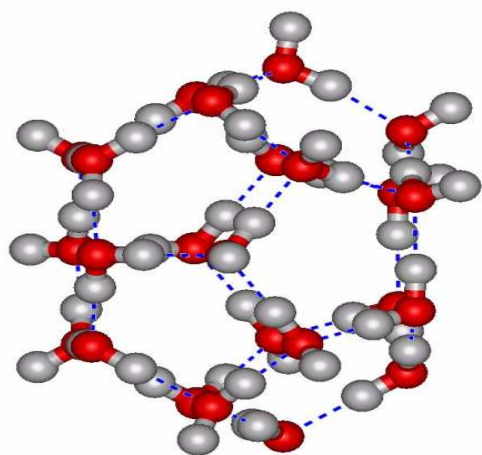

(b)

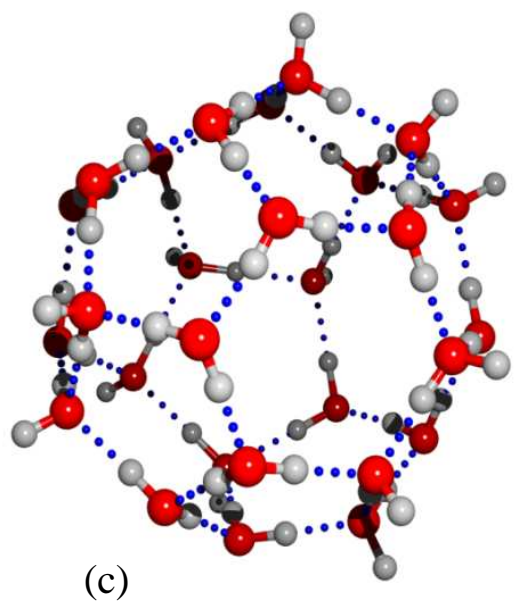

Figure 1.1 Cavities of hydrate structure: (a) pentagonal dodecahedron $\left(5^{12}\right)$, (b) Terakaidecahedron $\left(5^{12} 6^{2}\right),(\mathrm{c})$ Hexakaidecahedran $\left(5^{12} 6^{4}\right)$ 


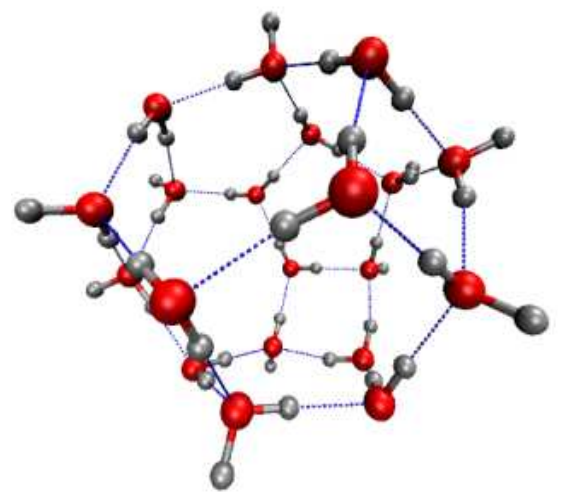

(a)

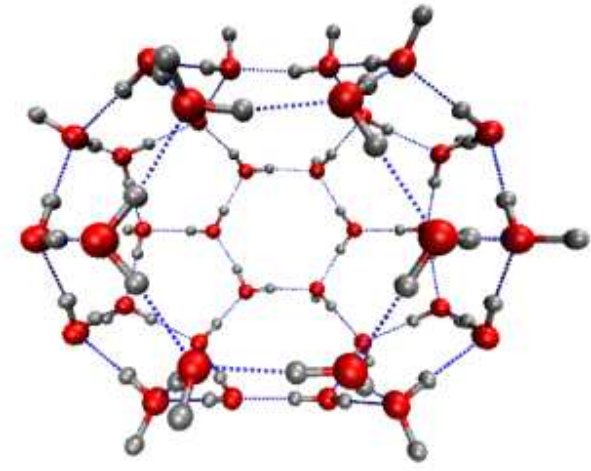

(b)

Figure 1.2 Cavities of hydrate structures (a) Irregular dodecahedron $\left(4^{3} 5^{6} 6^{3}\right)$, (b) Icosahedron $\left(5^{12} 6^{8}\right)$

Table 1.1 Hydrate crystal structure ${ }^{19}$

\begin{tabular}{cccccccc}
\hline Property & \multicolumn{2}{c}{ Structure I } & \multicolumn{2}{c}{ Structure II } & \multicolumn{3}{c}{ Structure H } \\
\hline Cavity & Small & Large & Small & Large & Small & Medium & Large \\
Description & $5^{12}$ & $5^{12} 6^{2}$ & $5^{12}$ & $5^{12} 6^{4}$ & $5^{12}$ & $4^{3} 5^{6} 6^{3}$ & $5^{12} 6^{8}$ \\
$\begin{array}{c}\text { Cavities/unit cell } \\
\quad 2\end{array}$ & 6 & 16 & 8 & 3 & 2 & 1 \\
$\begin{array}{c}\text { Water } \\
\text { molecules/unit } \\
\text { cell } \\
\begin{array}{c}\text { Average cavity } \\
\text { radius (̊) }\end{array}\end{array}$ & 3.95 & 4.33 & 3.91 & 4.73 & 3.94 & 4.04 & 5.79 \\
\hline
\end{tabular}

In structure I, each unit cell has 2 small and 6 large cavities. The small cavity is composed of 20 water molecules arranged to form 12 pentagonal faces $\left(5^{12}\right)$ and the resulting polyhedra is known as pentagonal dodecahedra. The large cavity contains 24 water molecules, which form 12 
pentagonal and 2 hexagonal faces $\left(5^{12} 6^{2}\right)$ and the polyhedra is tetrakaidecahedra. Structure I has total of 46 water molecules per unit cell and form the primitive cubic lattice with lattice constant of 12.0 A. The cavities of the structure I are shown in Figure 1.1. The ideal structural composition for a fully occupied structure $\mathrm{I}$ is $8 X \cdot 46 \mathrm{H}_{2} \mathrm{O}$ where $X$ is the guest molecule.

Structure II has sixteen $5^{12}$ cavities and eight $5^{12} 6^{4}$ (hexakaidecahedra), which is a 16 -sided cage, per unit cell. It has total of 136 water molecule per unit cell and form the face centre cubic lattice with lattice constant of $17.3 \AA^{21}$. The cavities of the structure II are shown in the Figure 1.2. The ideal structural composition for a fully occupied structure I is $24 \mathrm{X} .136 \mathrm{H}_{2} \mathrm{O}$ where $\mathrm{X}$ is the guest molecule.

Structure $\mathrm{H}$ hydrate was reported by Ripmeester et al. ${ }^{22}$ and the unit cell have 34 molecules with the composition: 3 cages of $5^{12}, 2$ cages of $4^{3} 5^{6} 6^{3}$ (irregular dodecahedron,) and 1 cage of $5^{12} 6^{8}$ (icosahedrons). The cavities of structure $\mathrm{H}$ are shown in Figure 1.4. Unlike structure I and structure II, which generally forms hydrate with single occupant either the small or large cavity the structure $\mathrm{H}$ requires two sizes of molecules to stabilize the structure. The properties of the structures are tabulated in Table 1. The lattice structure of structure I, structure II and structure $\mathrm{H}$ are shown in Figure 1.3, Figure 1.4 and Figure 1.5 respectively.

The presence of the guest molecule stabilizes the host lattice structure because of the relatively weak van der Waals interactions between the host water molecules and the entrapped guest molecules. There is no bonding between the guest and host molecules. Pure methane, ethane, carbon dioxide form the structure I hydrate and argon, oxygen form structure II hydrates. Mixture of gases forms either structure I or structure II or structure $\mathrm{H}$ depends upon the molecule size and the composition. Propane, methane and ethane mixture forms structure II hydrates. Gas hydrates 
are non-stoichiometric compounds since all available cages within the lattice structure are not completely occupied for stability.

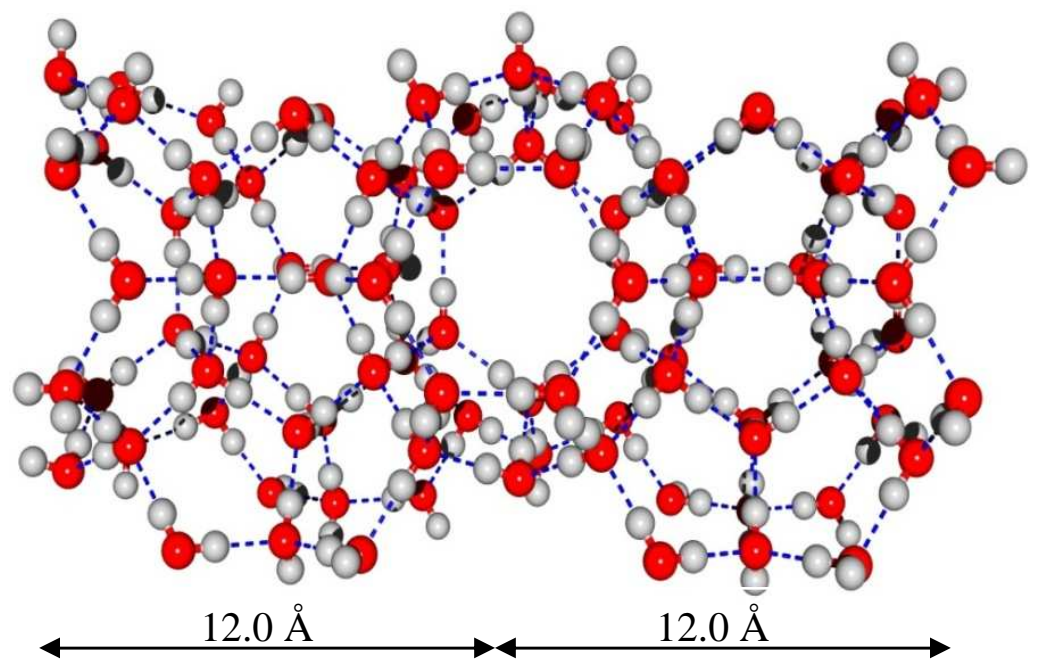

Figure 1.3 Structure I hydrate in 2 adjacent unit cells in $\mathrm{x}$-direction

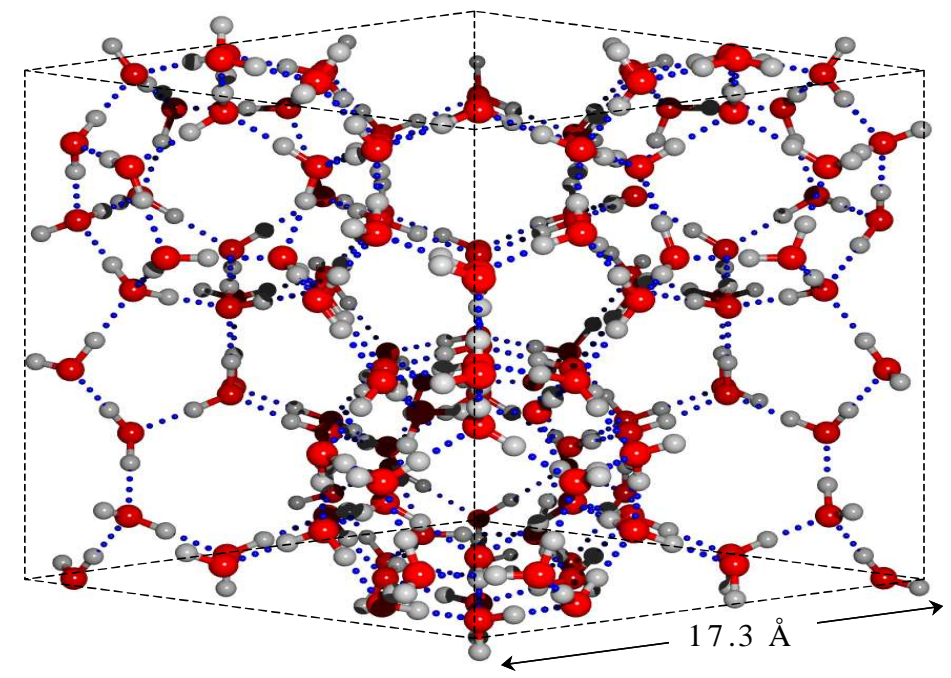

Figure 1.4 Structure II hydrate in one unit cell of cubic structure 


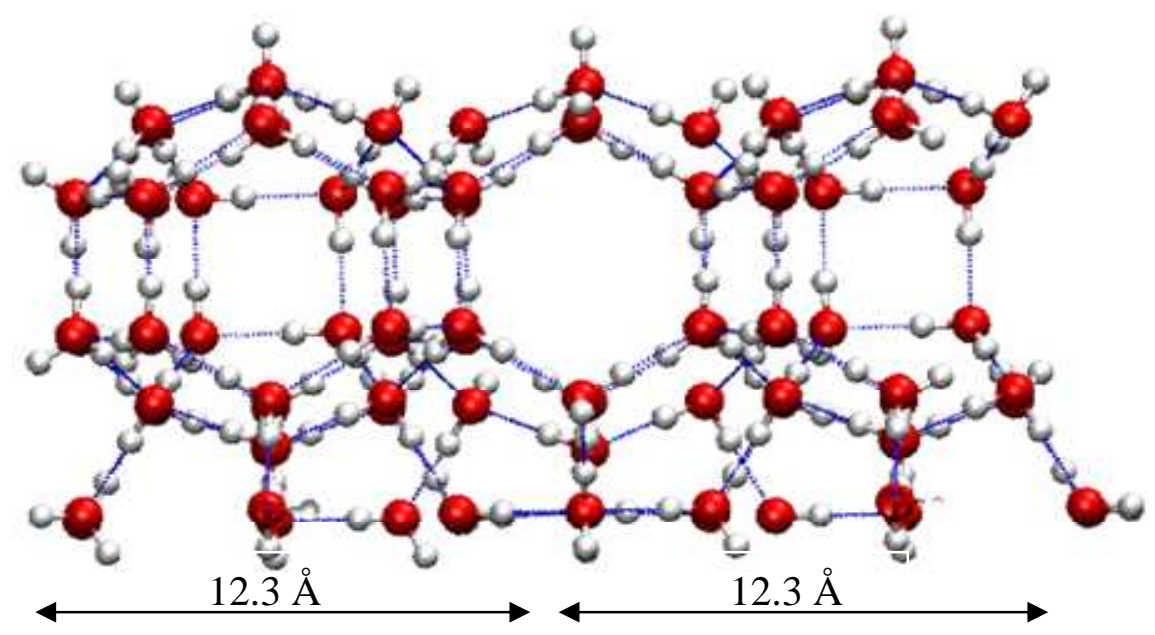

Figure 1.5 Structure $\mathrm{H}$ hydrate in 2 adjacent unit cells of hexagonal structure in $\mathrm{x}$-direction

\subsection{Stability}

Natural gas hydrates are stable at conditions of low temperatures and high pressures. The phase diagram for a methane hydrate in Figure 1.6 shows the combination of temperatures and pressures which mark the phase transition from a system of water/ice and methane hydrate to coexisting gas and water. The range of subsurface depths in which the prevailing temperature and pressure conditions keep a gas hydrate stable is called the hydrate stability zone (HSZ).. In the ocean, the hydrate stability zone starts at a depth of approximately 450 meters. The range of depths in which the temperature gradient is to the left of the phase boundary indicates the hydrate stability zone which is different for permafrost and ocean settings. This graph shows the hydrate stability zone for the ocean settings.

In addition to temperature and pressure, the composition of both water and gas are important to determine the stability of gas hydrates in specific settings. Natural subsurface environments exhibit variations in formation water chemistry, and these changes create local shifts 
in the phase boundary. Local conditions and the zone's geologic history determine whether the hydrates will occur within the stability zone. Higher salinity restricts hydrate formation causing the phase boundary in the Figure 1.6 to shift to its left. Similarly, the presence of other gases such as carbon dioxide, hydrogen sulfide and high molecular weight hydrocarbons will increase the stability of the hydrate, shifting the phase boundary to its right.

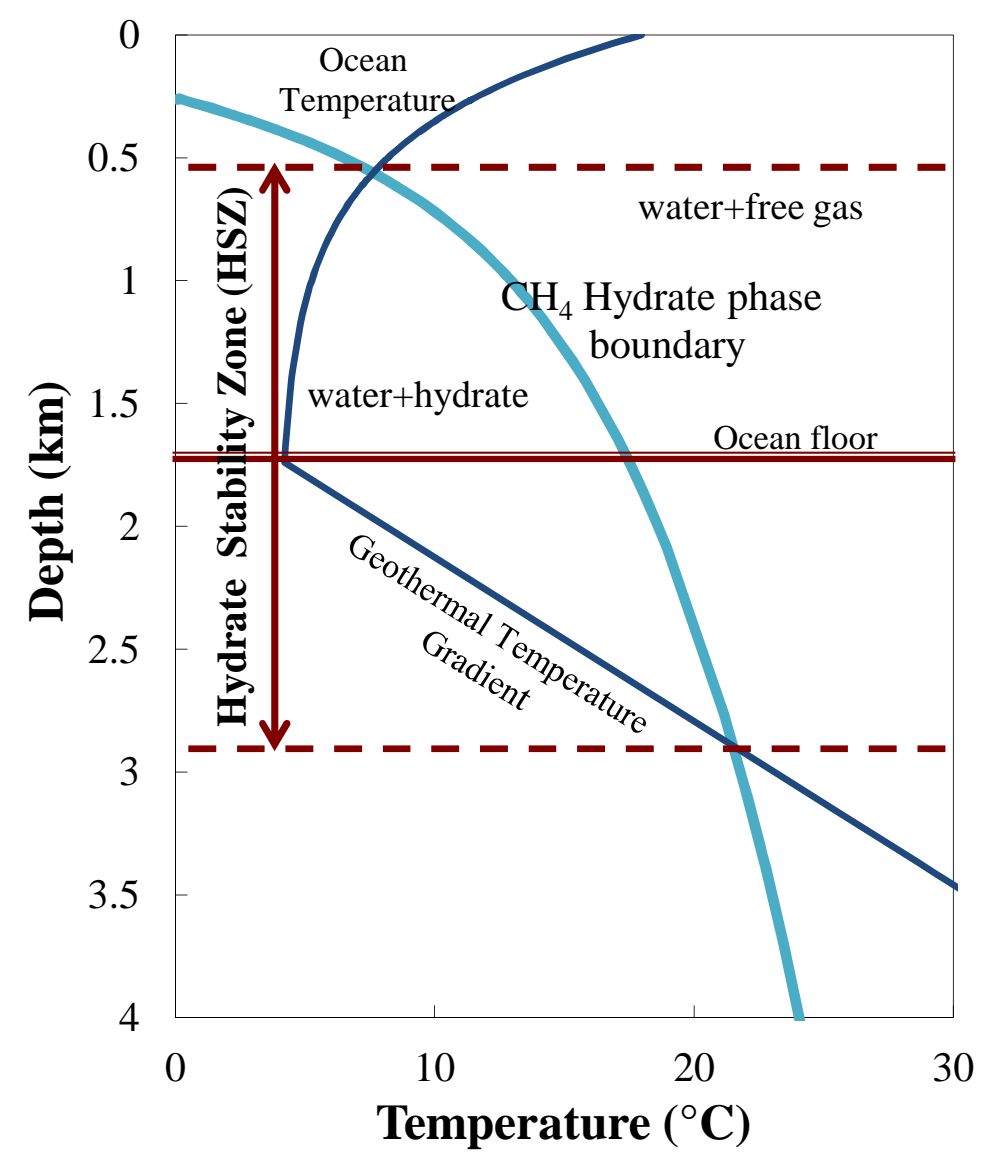

Figure 1.6 Phase diagram for methane hydrate showing hydrate stability zone (HSZ) in the ocean settings. Hydrates are formed in the HSZ, anything above or below destabilizes the hydrates in to water and free gas. 


\subsection{Motivation}

Gas hydrates formation conditions, low temperatures and high pressures are present in most of the world's oceans. Most of the natural hydrate reserves are found in the marine environment, an order of two times than the permafrost hydrate reservoir ${ }^{10,12,23}$. Along with the temperature and pressure sufficient amount of guest gas also has to be present to form gas hydrates. When gas in the hydrate is produced by bacterial transformation of organic matter the gas formed is mainly methane leading to form sI hydrates ${ }^{24}$. This is the most common hydrate type in nature. However, at higher temperatures, greater than $373 \mathrm{~K}$, thermo-catalytic reactions produce thermogenic gases. These gases are heavier than methane like ethane, propane, etc. Due to the presence of higher hydrocarbons the hydrates formed are structure II hydrates. These complex hydrates are common in the Cascadia Margin ${ }^{25-26}$, Gulf of Mexico ${ }^{27}$ and Barkley Canon ${ }^{28}$. Naturally occurring structure $\mathrm{H}$ hydrates also exits at Barkley Canon $^{28}$. Large amount of free gas are estimated to exit below the hydrate stability field ${ }^{29}$, the destabilization of these mixed gas hydrates under sea floor is believed to trigger slump slides which can result into the release of large amount of gas into the water column. So, a study of the stability of these naturally occurring hydrates in water needs a special attention.

\subsubsection{Dissolution}

It is important to understand the phenomena governing the evolution of gas hydrates in water to predict their stability. When temperature and pressure changes of the ocean occurring as a result of the changing climate could be a factor affecting the stability of hydrates, difference in chemical potentials of the gas forming hydrate in the hydrate and water phases also influence the stability of gas hydrates. When the instability of hydrates is caused by the existing temperature and 
pressure conditions, then the phenomenon of decomposition of hydrates can be referred as dissociation $^{18}$. In this case, the hydrate is said to be outside the hydrate stability zone. But, when hydrate is inherently stable at the existing temperature and pressure conditions, then the concentration differences of the gas forming hydrate between the hydrate and the liquid phase if any, initiates the decomposition of hydrate. This phenomenon can be referred as dissolution and it is a slower process compared to dissociation ${ }^{18}$. Typically, the concentration of the guest gas in the ocean is significantly lower than its solubility in seawater. Thus, when a hydrate is exposed to ocean water, both hydrate and the water phases are not in equilibrium with respect to the concentrations of the corresponding gas. This can result in dissolution of these gas hydrates in to the water ${ }^{30}$. Outcrops of hydrates in the Gulf of Mexico and Pacific Oceans are found on the seafloor $^{31}$ and these outcropping hydrates are vulnerable to dissolution. The majority of hydrate dissociation studies were performed focusing on heat-transfer controlled systems, relevant to oil and gas pipeline blockages ${ }^{32}$. However, there has been little work done on hydrate dissolution and that is too focusing on structure I methane or carbon dioxide hydrates. To the best of my knowledge, no work has been done on dissolution of hydrates that include mixed higher hydrocarbon hydrates like methane-ethane-propane mixed hydrates. This work is an attempt to understand the dissolution process of the mixed hydrates under deep water conditions and the factors affecting it such as the gas concentration in the hydrate phase.

There also exits large amount of free gas below the hydrate stability field (HSF), any instability in the gas hydrates causing slump slides will release large amount of gas in to the water column below the HSF. In some cases production and exploration activities like imminent blow-out and oil leaks will release the massive free gas in to the water column. These released hydrocarbons dissolves in water and can cause significant effects on the ecosystem. One way to know the impact 
of a release of hydrocarbon from the deepwater is to model the processes which govern their behavior in such releases to predict their impacts. Releasing of natural gas from the sea floor to the water column within the HSF is a natural and widely occurring process ${ }^{33}$. The released gas travels upwards as it is lighter than water. Due to lower temperatures and high pressures in the deep water, in the hydrate stability field, a hydrate skin is formed at the bubble water- interface and slows down the gas bubble dissolution in the undersaturated open ocean. ${ }^{34}$ As the gas bubble reaches low pressure, outside the hydrate stability field, hydrates decompose into water and gas. Hydrate formation and decomposition significantly affect the buoyancy of the jet plume ${ }^{18}$. Understanding the behavior of free and dissolved gases in the hydrate formation and stability is important to correctly make decisions and predictions of the fate of the gas bubbles rise in case of blowouts. The BP incident in the Gulf of Mexico failure of the large 100-ton cofferdam containment structure is believed to be due to the hydrate formation within it. This demonstrates that the role of hydrates under deep water conditions still is inadequately understood ${ }^{35}$. The best current plume models only account for structure I hydrates (pure methane), but natural marine hydrocarbons form mixed hydrates. The presence of higher hydrocarbons forms structure II hydrates ${ }^{36}$, which are stable at far lower pressures. Current models do not account for hydrate structures other than simple type structure I methane hydrates and therefore would not predict the undersea plume buoyancies observed for the BP spill. Also important in understanding the fate of the plume is the flow field around the bubble, a variety of parameters affects the flow field parameters, which determines the fluid transfer across the interface. In addition to hydrodynamic variables, thermodynamic variables also have to be incorporated to assess the flux of the natural gas in to the ocean ${ }^{33}$. The driving force for the gas bubble is the concentration difference between saturation and the bulk phase. The saturated concentration is nothing but the solubility of the hydrocarbon in the water phase. The 
aqueous hydrocarbon solubilites are different when there is hydrate formation on the gas bubble (HSF) and when there is no hydrate formation. Within the HSF, the hydrocarbon solubility in water is determined by the chemical potential equilibrium between the hydrate phase and liquid water phase. Outside the HSF, the solubility of hydrocarbon is determined by V-L equilibrium ${ }^{33}$. This information is needed for the process of gas hydrate metastability and formation in accidentally released well fluids.

\subsubsection{Experiments at NETL, Pittsburgh}

To understand the behavior of the free and dissolved hydrocarbons and their role in the hydrate formation and stability, the National Energy Technology Laboratory (NETL) at Pittsburgh has conducted experiments to study the stability and formation of hydrates under deep water conditions. The experiments were carried out in an existing High-Pressure Water Tunnel Facility (HWTF) at NETL by Robert Warzinski's group. The schematic of the HWTF is given in Figure 1.7. The HWTF is designed to operate for $\mathrm{CO}_{2}$ and natural gas components at pressures up to 34.5 $\mathrm{MPa}$ and temperatures near freezing to $90{ }^{\circ} \mathrm{C}$. They have used the HWTF to study the formation and behavior of carbon dioxide hydrate particles in the past ${ }^{37-38}$. Similarly, experiments with pure methane gas in the HWTF were carried out with pressures ranging from $3 \mathrm{Mpa}$ to $30 \mathrm{MPa}$ and temperatures ranging from $2-12{ }^{\circ} \mathrm{C}$. These conditions are similar to deepsea conditions. Experiments with natural gas components (methane/ethane/propane) mixture were used to study the formation of structure II hydrates. The hydrocarbon mixture composition used is $87.5 \%$ methane, $8.1 \%$ ethane and $4.4 \%$ propane. This composition of the mixture is similar to that was found in the

Macondo reservoir in the Gulf of Mexico ${ }^{39-40}$. Experiments were conducted for fresh water and artificial sea water for pure methane and for the methane-ethane-propane mixture. 


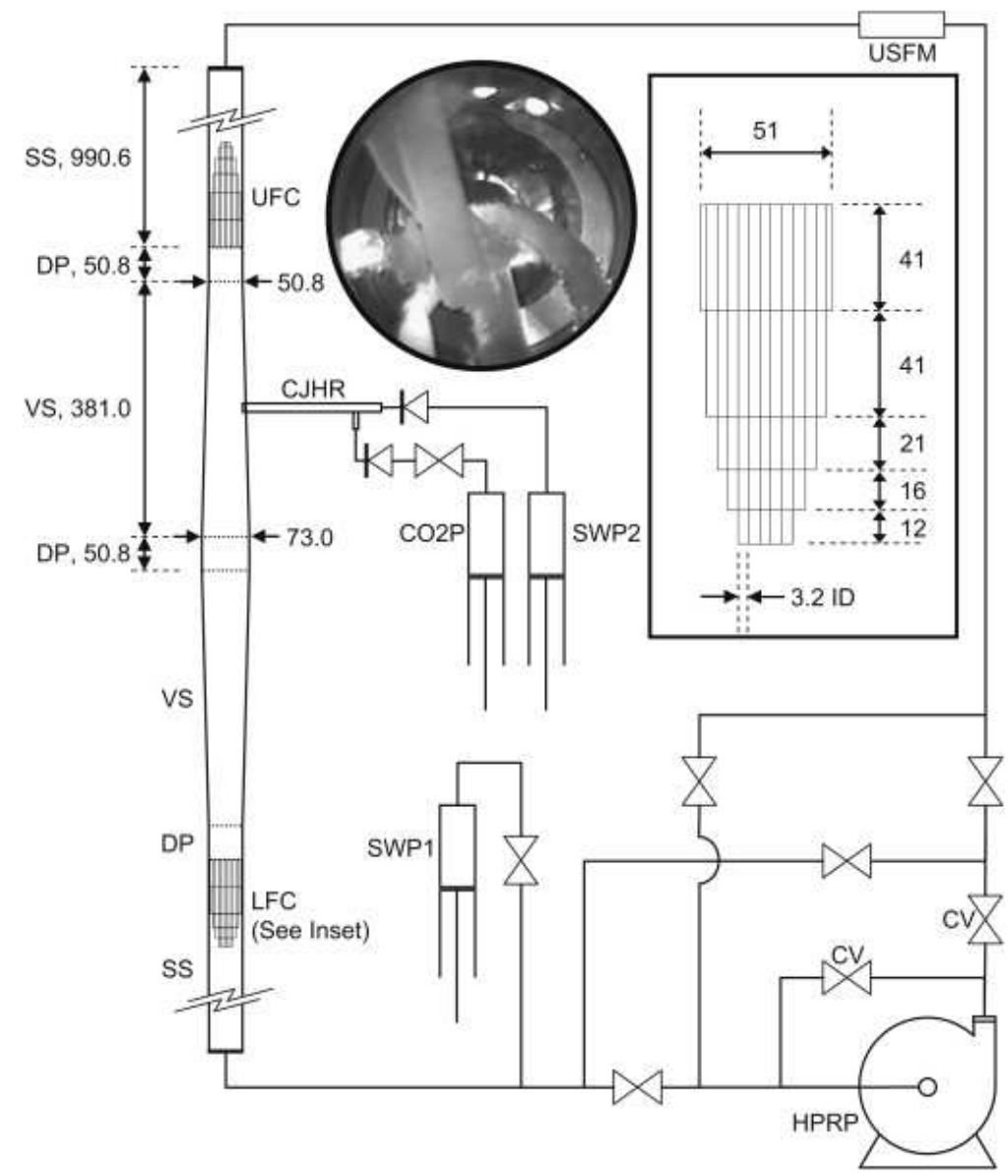

Figure 1.7 Schematic of the high pressure water tunnel facility (HWTF) at NETL, Pittsburgh. The high pressure water tunnel (HWT) is shown on the left side. Detail description about the apparatus can be found in Warzinski et al. $(2008)^{37}$.

In summary, gas hydrate mounds in contact with under-saturated ambient sea water dissolve over time. This is due to the chemical potential difference between the hydrate and the surrounding water $^{30}$. Naturally occurring hydrates are not simple hydrates but mostly contains higher hydrocarbons like ethane, propane, etc., which form sII hydrates. This work is an attempt to understand the presence of higher hydrocarbons like ethane and propane on the dissolution of hydrates. In addition, understanding the thermodynamics of the mixed hydrates at the two phase hydrate equilibrium under deep water conditions is essential to model better the natural gas in the hydrate stability field. 


\subsection{Overview of Previous Work}

\subsubsection{Experiments on dissolution of gas hydrates}

There have been fewer in-suite experimental studies on dissolution of hydrates. The first insitu experiment known to be done on dissolution of hydrates in the ocean was by Rehder et al. at $1000 \mathrm{~m}$ depth in the Monterey Bay ${ }^{30}$. They measured the dissolution rates of pure synthetic samples of $\mathrm{CH}_{4}$ and $\mathrm{CO}_{2}$ hydrates exposed directly to under saturated sea water conditions of $3.6{ }^{\circ} \mathrm{C}$ and 104.8 bar. The temperature and pressure conditions are within the hydrate stability field. They found that methane hydrate dissolve much slower compared to $\mathrm{CO}_{2}$ hydrate. They proposed a diffusive boundary layer model for the dissolution process, the kinetics of which is mass transfer controlled. They concluded this because the rate of dissolution was proportional to the individual gas solubilities. The mass transfer occurs due to the diffusion of the molecules of the guest species from the hydrate boundary to the bulk of the ocean.. The ratio of dissolution rates of $\mathrm{CO}_{2}$ hydrate to methane hydrate was measured to be 11 , which was comparable to the solubility ratio of $\mathrm{CO}_{2}$ to methane in water. The solubility ratio of $\mathrm{CO}_{2}$ to methane was calculated at the same conditions of temperature and pressure to be 10.4 .

Hester et al. $^{41}$ performed the dissolution studies of natural hydrates on the sea floor at Barkley Canyon $\left(850 \mathrm{~m}\right.$ depth and $\left.4.17^{\circ} \mathrm{C}\right)$. They considered two types of multi-component hydrates, a "white" hydrate and a "yellow" hydrate stained with condensate oil. These multicomponent hydrates contain methane and higher hydrocarbon. The white hydrate contains mixture of structure I and structureII hydrates and yellow hydrate contains mainly structure II with small amounts of structure H. In their study they observed that the white hydrate dissolve faster than the yellow hydrate. They showed that mass transfer is the rate controlling mechanism for dissolution of these natural hydrate outcrops. To test their correlation for convective mass transfer, they compared 
to the experimental results of Rehder et al. ${ }^{30}$ synthetic hydrates using water velocities as the fitting parameters. The correlation agreed well to the synthetic hydrates, and when applied to the natural hydrates, the mass transfer correlation predicted the dissolution rate within $20 \%$. The complex natural hydrates are more stable than that predicted by the dissolution models ${ }^{30,42-43}$. To describe the dissolution of the natural complex hydrates, with highly heterogeneous morphology and composition, proper hydrodynamics will be needed in the dissolution models.

Nihous and Masutani ${ }^{44}$ used the data of Rehder et al. ${ }^{30}$ in their model and questioned the idea that the hydrates are only stable when surrounded by water that is saturated with methane, since calculations failed to mirror the observed proportionality of decomposition rate and solubility for $\mathrm{CH}_{4}$ and $\mathrm{CO}_{2}$ hydrates. Bigalke et al. ${ }^{45}$ measured the dissolution rates of in-situ generated pure methane hydrates exposed to precisely adjusted friction velocities in a series of laboratory-based experiments at pressure and temperature conditions within the hydrate stability field. They showed from the experiments that hydrate decomposition is an entirely diffusion-controlled process under the conditions investigated. If the buried hydrate dissolution is diffusion controlled and they are thermodynamically stable then the sediments surrounding the hydrate deposits should have nearsaturation guest gas concentrations. Lapham et al. ${ }^{43}$ measured the dissolved methane concentration in pore-waters surrounding outcropping hydrate and showed that pore-fluids are greatly undersaturated with respect to expected values for equilibrium with methane gas hydrate. The dissolution rates calculated from their in situ $\mathrm{CH}_{4}$ concentration gradients are significantly less than the dissolution rates predicted from dissolution rate kinetics. They suggested that the meta-stability of hydrates is due to the diffusion retarding factors found naturally in ocean sediments such as oil coatings or bio films which enhances the stability of the out cropping hydrates. 


\subsubsection{Dissolution models}

Several dissolution models were investegated on to study the dissolution and or convective diffusion of $\mathrm{CO}_{2}$ or methane hydrate layer gas in the ocean. A simple heterogeneous model was proposed by Gabbito et al ${ }^{46}$ to explain the dissolution of hydrate-coated $\mathrm{CO}_{2}$ in water, in which there is a simultaneous hydrate formation at the $\mathrm{CO}_{2}$ hydrate interface and hydrate dissolution at the hydrate-water interface. They indicated that the rate controlling step for the dissolution process is either mass transfer within the hydrate phase or a dissolution reaction in the hydrate-water interface. This model was applied to the data obtained from experimental work by Brewer et al. ${ }^{47}$ on a small amount of liquid $\mathrm{CO}_{2}$ released at $800 \mathrm{~m}$ open ocean depth. The calculations showed that the dissolution rate depends on the mass transfer coefficient of $\mathrm{CO}_{2}$ within the hydrate. It was also found that the mass transfer within the hydrate phase depends on the flow condition outside the hydrate layer, which was not considered in the model. The model predicts the complete disappearance of the $\mathrm{CO}_{2}$ hydrate layer, which is contrary to that observed in the experiments of Brewer et al. ${ }^{47}$ Similar results were found by Shindo et al. ${ }^{48}$ by calculating dissolution reaction rate at the hydrate-water interface. Mori and Murakami ${ }^{49}$ made a critique of a model due to Gabbito et al. ${ }^{46}$ for estimating the hydrate film thickness. They concluded that the hydrate film thickness values predicted by this model are highly questionable and should not be used as the basis of considering the mechanism for the interphase $\mathrm{CO}_{2}$ transfer across hydrate films.

Another elementary model has been proposed by Nihous and Masutani ${ }^{44}$ to explain the dissolution of pure hydrate in undersaturated seawater. The model is based on a combination of a decomposition reaction within a desorption layer surrounding the hydrate and diffusion of the gas molecules through a diffusive boundary layer. They suggest that the concentration of the guest species in water plays an important role in providing the necessary fugacity gradient for the 
dissolution. They applied this model to the methane hydrate data obtained from the experiments by Rehder et al. on dissolution rates of methane and $\mathrm{CO}_{2}$ hydrates in undersaturated seawater on the seafloor. The model predicted that desorption layer should be undersaturated with respect to methane for the dissolution to proceed. The concentration of methane in desorption layer was approximately found to be approximately half the equilibrium solubility of methane in the ambient water. But, the model could not explain deep sea observations related to the dissolution of $\mathrm{CO}_{2}$ hydrates. The model predicted a much smaller degree of undersaturation in the desorption layer with respect to $\mathrm{CO}_{2}$ when compared to experimental observations. All these models could make closer predictions to explain the behavior of methane and $\mathrm{CO}_{2}$ hydrates in undersaturated seawater

Exposed hydrate mounds present on the sea floor in under-saturated conditions should be dissolving. But the observed rates of dissolution appear to be an order of magnitude lower than the dissolution rates obtained from the dissolution models ${ }^{43}$. This means that the seafloor methane hydrate outcrops have been found to show considerably longer lifetimes than that predicted by all the dissolution models. Also, the slow rate of dissolution of methane hydrate reported by Rehder et al. $^{30}$ from their in situ experiments implies that the longevity of sea floor methane hydrate outcrops observed today should be possible only by the supply of sufficient $\mathrm{CH}_{4}$ to maintain boundary layer saturation or a continuous hydrate regrowth. This is called the "push-up-pop" model ${ }^{50}$ whereby hydrates remain stable by dissolving at the surface and at the same rate forming within subsurface sediment. If this is true then the sediments surrounding the surface hydrate deposits should have elevated methane concentrations, but the measurements of methane concentration in sediments near the hydrate surface shows that pore fluids are greatly under-saturated with respect to expected values for equilibrium to methane hydrate ${ }^{43}$. It is necessary to understand the factors affecting the dissolution phenomenon to assess the long term stability of hydrate deposit. It is yet to be known 
why there is a difference between the theoretical model predictions and the field observations in the sea. There might be some diffusion retarding factors that stabilizes the natural hydrates. However, it can be understood that the concentration of the gas forming hydrate in both water and hydrate phase has a key role in providing the necessary driving force for the dissolution of their hydrates. It is required to study how the presence of higher hydrocarbons, like propane, ethane, which forms structure II hydrates effects the dissolution behavior of the mixed hydrates and so the stability of hydrates.

\subsubsection{Dissolution and dissociation studies using MD simulations}

Molecular dynamic (MD) simulations have proved to be useful for predicting various properties of methane hydrate. Performing the experimental study of these hydrates are expensive and tedious, MD simulation can be used to understand the microscopic structures of gas hydrates and its correlation to macroscopic properties. English et al. ${ }^{51}$ used MD simulations to validate the experimental values of thermal conductivity determined by same study. They were also used to evaluate the possible reason for a lower thermal conductivity of methane hydrate compared to ice ${ }^{52}$. There have been several studies on hydrate formation, nucleation and dissociation and also on phase interface behavior of hydrate and water using MD and Monte Carlo simulations ${ }^{51,53-55}$. There have been a limited number of molecular dynamic investigations into hydrate dissociation and dissolution in particular, and most of them were done on methane hydrate and carbon dioxide hydrate.

Baez and Clancy ${ }^{53}$ carried out MD simulations to study the dissolution of spherical methane hydrate crystal in the presence of water using non-polarizable SPC/E model for water at $270 \mathrm{~K}$ and 40 bar, where the hydrate crystallite of radius $12 \AA$ had around 250 molecules of water and 32 molecules of methane in the hydrate phase. They found that the crystallite dissolved in 146 ps and 
the melting occurred in a stepwise manner. The interface formed between the hydrate crystal and the surrounding liquid phase consisted mainly of partial cages which survived for long times keeping the structure stable before it dissolved completely. They also noted that the size of the crystallite, initially or during the dissociation, did not affect the rate of dissociation. English et al. ${ }^{51}$ also studied dissolution of spherical hydrate crystallites in water at $277 \mathrm{~K}$ and 68 bar using TIP4PFQ water model. The crystallites were found to dissociate within $400 \mathrm{ps}$. The dissolution rates were found not to be affected by the methane composition of the hydrate phase between $80-100 \%$, but the empty hydrate clusters were found to dissolve rapidly. The size of the crystallite or the size of the liquid phase was not found to influence the dissolution rates, but an increase in the size of the liquid phase reduced the initial delay in the dissolution process.

It has to be noted that the temperature and pressure conditions at which the simulations were carried out by both Baez et al. ${ }^{53}$ and English et al. ${ }^{51}$ are within the hydrate stability zone. Although the liquid phase considered is unsaturated with respect to methane, hydrate dissolution caused by the concentration difference of methane between the hydrate and the liquid phases is supposed to be a slow process and cannot occur in the very short timescales observed by these authors. So, it is more likely that the dissolution occurred because the crystallites considered were subcritical in size. The critical size of a hydrate cluster for nucleation has been estimated by Radhakrishnan et al. ${ }^{54}$ to be between 10-14 $\AA$ in a linear dimension. Therefore, the size of the hydrate phase has to be more than the critical size of nucleation to remain stable and have a continuous growth.

Rodger et al. ${ }^{55}$ studied the behavior of methane hydrate-methane gas interface at temperatures 15-20 K above the theoretical melting temperature using SPC water model. Analysis based on a new set of order parameters to identify the water in different phases helped them observe ice and hydrate like structures in the water phase, but clustering of these structures was not 
observed. Myshakin et al. ${ }^{56}$ performed molecular dynamic studies on methane hydrate dissociation at $277 \mathrm{~K}$ and 68 bar. They observed that the dissociation rate depends on the hydration number and found that a decrease in cage occupancy from $100 \%$ to $95 \%$ causes a greater change in the dissociation rate than for a decrease from $95 \%$ to $85 \%$. They note that the presence of empty cages destabilizes the hydrate lattice and stimulates the dissociation process. They state that the decomposition rates of the hydrate lattice are constant during the first several nanoseconds and then demonstrate oscillating behavior. The oscillatory behavior was attributed to a regrowth of the hydrate cages at the interface. In most of the above simulations done on dissociation or dissolution of hydrates in to water, there has been a mention of possible regrowth of the hydrate clusters due to the presence of the hydrate-like water structures in the liquid phase, which is referred as the memory effect. Such phenomena also increase the longevity of a hydrate.

Hirai et al. ${ }^{57}$ studied the stability of $\mathrm{CO}_{2}$ hydrate using $\mathrm{MD}$ simulations using an interatomic potential function based on a model given by Kumagai et al. ${ }^{58}$ for both $\mathrm{CO}_{2}$ and water. They found that it is unstable compared to both empty and Argon clathrate hydrates. This was attributed to the destabilizing effect caused by the repulsive force acting between the $\mathrm{O}$ atoms of $\mathrm{CO}_{2}$ and $\mathrm{O}$ atom in water on the lattice structure. Kvamme et al. ${ }^{59}$ performed MD simulations to study the $\mathrm{CO}_{2}$ hydrate-water interface at conditions within the hydrate stability zone using SPC/E water model. They evaluated the steady state interface thickness using different analyses like the hydrogen density profiles and radial distribution functions. Sarupria and Debenedetti ${ }^{60}$ studied the $^{2}$ effect of cage occupancy on the rate of hydrate dissociation of $\mathrm{CO}_{2}$ hydrate in contact with liquid water using the Trappe force field ${ }^{61}$ for $\mathrm{CO}_{2}$. They concluded that the dissociation is dependent not only on overall cage occupancy, but also on the cage specific (small and large cages) occupancy. 
Most of molecular simulations for the stability of gas hydrates were done on pure methane hydrates or on pure carbon dioxide hydrates. There are few mixed hydrate simulations based on $\mathrm{CO}_{2}$ exchange with methane hydrates or on the stability of $\mathrm{CO}_{2}-\mathrm{CH}_{4}$ hydrates. All these guest molecules form sI hydrates, but there are naturally occurring complex mixed hydrates which forms sII and sH hydrates. To the best of our knowledge, the dissociation and dissolution of sII hydrates have not been studied using MD simulations.

\subsubsection{Solubilities of hydrocarbon in HSF}

Gas bubbles are released into the hydrate stability field due to the natural sweeps or due to the imminent blow out, which is a natural and widely seen process. These gas bubbles within HSF forms a hydrate rim affecting the dissolution of gas in to the sea water ${ }^{34}$. Maini and Bishonoi ${ }^{62}$ showed in lab experiments that a hydrate skin at the bubble-water interface is formed for the water saturated with methane. Rehder et al. ${ }^{34}$ released methane and argon bubbles within the hydrate stability zone and they observed the hydrate rim formation on the methane bubble. They observed a $12 \%$ slower initial dissolution rate of methane bubbles released in HSF compared to those released above the HSF. Understanding the behavior and a better knowledge of hydrate-liquid water $\left(H-L_{w}\right)$ two phase equilibrium is necessary for studying the formation and accumulation of subsea hydrates ${ }^{63}$, formation of hydrate skin on the bubble, modeling the dissolution rates of hydrates in under-saturated surrounding environment ${ }^{33,64}$. The driving force for gas bubble dissolution with/without hydrate is the concentration difference between the bulk gas concentration and the gas solubility at the interface. Thus, the understanding of gas solubility is essential for accurate modeling of the dissolution rate. The hydrate former solubility in pure water dependency on temperature and pressure in the liquid-water equilibrium region $\left(\mathrm{L}_{\mathrm{w}}-\mathrm{V}\right)$ are different from the $\mathrm{H}-\mathrm{L}_{\mathrm{w}}$ equilibrium region ${ }^{65-66}$. The solubility of hydrate former gas in water in the $\mathrm{L}_{\mathrm{w}}-\mathrm{V}$ equilibrium 
decreases with increase in temperature at a given pressure, while in the $\mathrm{H}-\mathrm{L}_{\mathrm{w}}$ region the solubility in pure water increases with increase in temperature ${ }^{66-69}$.

Most of the available experimental data on hydrate equilibrium is on three phase $\mathrm{H}-\mathrm{L}_{\mathrm{w}}-\mathrm{V}$ equilibrium. There is a limited amount of experimental data available in the literature for the solubility of aqueous hydrocarbon solubility at two phase hydrate-water equilibrium. Aqueous $\mathrm{CO}_{2}$ solubility at the two phase $\mathrm{H}-\mathrm{L}_{\mathrm{w}}$ equilibrium is a research hot topic because of the growing interest in the sequestration potential of the $\mathrm{CO}_{2}$ in the marine environments. Recently, there is an increase in the interest for the solubility of natural gas in the subsea environments.

Yang et al. ${ }^{70}$ measured the solubility of methane in water at $\mathrm{H}-\mathrm{L}_{\mathrm{w}}$ equilibrium in the pressure range from 2 to $20 \mathrm{MPa}$ and in the temperature range of $273-285 \mathrm{~K}$. Kim et al. ${ }^{69}$ modified the experimental apparatus of Yang et al. ${ }^{70}$ to determine the solubility of methane in water at hydrate-liquid water equilibrium. Methane solubilities were measured in the temperature range of 276-282 K and in the pressure range of 5 to $20 \mathrm{MPa}$. Servio and Englezos measured the solubility of $\mathrm{CH}_{4}$ in liquid water in the presence of gas hydrate at temperatures varying from $274 \mathrm{~K}$ to $285 \mathrm{~K}$ and pressures range from 35 bar to 65 bar. Seo and Lee ${ }^{71}$ calculated the methane solubility for four temperature set from $274.15 \mathrm{~K}$ to $286.15 \mathrm{~K}$ and pressures from 6.0 MPa to 20 MPa. They have also determined the hydration number of methane using Raman spectroscopy at $10 \mathrm{MPa}$ and $274.15 \mathrm{~K}$ was found to be 6.0 . Kim et al. ${ }^{72}$ using the indirect method calculated the solubility of pure methane, carbon dioxide and ethane in aqueous solution at $\mathrm{H}-\mathrm{L}_{\mathrm{w}}$ equilibrium. They have also studied the effect of salt concentration on the solubility of these gases in the aqueous phase in equilibrium with hydrate phase. Lu et al. ${ }^{73}$ determined aqueous methane concentrations in equilibrium with sI hydrate by in situ Raman spectroscopy from temperature range of $276.6 \mathrm{~K}$ to 
294.6 K and pressures at 10-40 MPa. They also gave an empirical equation for aqueous solubility derived from the experimental data as:

$$
X\left(\mathrm{CH}_{4}\right)=\exp (11.0464+0.023267 P-(4886.0+8.0158 P) / T
$$

where $P$ is pressure in $\mathrm{MPa}$ and $T$ is temperature in $\mathrm{K}$.

There is very limited experimental data on aqueous ethane solubility at two phase $\mathrm{H}-\mathrm{L}_{\mathrm{w}}$ equilibrium. The experimental data is from Kim et al. ${ }^{69,72}$ and Yang et al. ${ }^{70}$ for temperatures in the range of $277-280 \mathrm{~K}$ and pressures in the range of 5-151 MPa. There is only one experimental data set reported in the literature for aqueous propane solubility at two phase $\mathrm{H}-\mathrm{L}_{\mathrm{w}}$ equilibrium. ${ }^{74}$ To our knowledge there is no reliable experimental data on the solubility of hydrocarbon mixture (methane-ethane-propane) components in the aqueous phase at $\mathrm{H}-\mathrm{L}_{\mathrm{w}}$ equilibrium.

There have also been some attempts to develop a model to predict the solubility of hydrate forming gas in the $\mathrm{H}-\mathrm{L}_{\mathrm{w}}$ equilibrium, but these models are for estimating the solubility of pure hydrocarbon gas in water. ${ }^{65,67,75-77}$. Hashemi et al.$^{65}$ developed the model for the calculation of methane solubility in water at $\mathrm{H}-\mathrm{L}_{\mathrm{w}}$ equilibrium based on the van der Waals and Platteeuw model. They have used the Trebble-Bishnoi equation of state for fugacity calculation. In their model, they have to readjust the reference chemical potential and reference enthalpy difference parameters with three phase vapor-liquid water-hydrate $\left(\mathrm{VL}_{\mathrm{w}} \mathrm{H}\right)$ equilibrium experimental data to improve the accuracy of the model. It was shown for the $\mathrm{VL}_{\mathrm{w}} \mathrm{H}$ phase equilibrium calculations that when the parameters are fitted to the experimental data the model works well in the range of the fit, but fail when extended outside the range of fit and also for mixed hydrates ${ }^{78}$. Mohammadi and Richon' ${ }^{77}$ model for $\mathrm{H}-\mathrm{L}_{\mathrm{w}}$ equilibrium used the Langmuir constant values from the equation of Parrish and Praustniz ${ }^{79}$ for the evaluation of the fugacity of water in the hydrate phase based on the van der Waals and Platteeuw model. The Langmuir constant, which accounts for the guest-host 
interactions, is an important term in the van der Waals and Platteeuw model. Over the years, there has been a lot of improvement in the accuracy of the Langmuir constant calculation: including guest-host interactions beyond the first shell, evaluation of full configurational integral and more realistic guest-host intermolecular potentials ${ }^{80-82}$. Parrish and Praustniz used the spherical core Kihara-type potential to describe the interactions between the guest and the surrounding water molecules, these Kihara potential parameters were obtained by empirical fit to hydrate equilibrium pressures. However, these parameters set of Parrish and Praustniz failed to accurately predict equilibrium pressures for different gas mixtures and temperature ${ }^{82-84}$. These Langmuir constants used by Mohammadi and Richon ${ }^{77}$ in their model for $\mathrm{H}-\mathrm{L}_{\mathrm{w}}$ equilibrium calculations may attribute for the deviation to the experimental data. Sun and $\operatorname{Duan}^{76}$ predicted the solubility of methane in water at $\mathrm{H}-\mathrm{L}_{\mathrm{w}}$ equilibrium using the van der Waals and Platteeuw model and angle dependent $a b$ initio intermolecular potentials. Their model prediction of methane solubility is as good as our model, but they did not predict for ethane and propane and also for the mixed hydrates in the $\mathrm{H}-\mathrm{L}_{\mathrm{w}}$ region. There are also some other models for $\mathrm{H}-\mathrm{L}_{\mathrm{w}}$ equilibrium ${ }^{69-70,85}$, but these models require considerable efforts to fit experimental data. Naturally occurring hydrates are not pure simple hydrates, they form mixed hydrates with the presences of higher hydrocarbons. Accurate knowledge of solubility of hydrocarbon mixture in water in the hydrate stability zone (HSZ) plays an important role to understand the stability of gas hydrates. ${ }^{30,33}$ 


\subsection{Objectives and Significance}

The overall objective of this thesis is to understand better the stability of the mixed hydrates, and the formation and stability of the gas bubbles that form complex hydrates under deep water conditions. Specifically, the following tasks were performed:

1. Molecular dynamics studies were carried out on sII hydrates in contact with water phase by changing the concentration of hydrate forming gas in the hydrate phase.

2. The effect of concentration of propane and ethane in the hydrate phase on hydrate stability was studied

3. A thermodynamic model to calculate the solubility of pure methane, pure ethane and pure propane in fresh water at two phase hydrate-water equilibrium was developed.

4. The methane-ethane-propane mixture solubility in water in equilibrium with sII hydrate in the absence of vapor phase was calculated

5. The aqueous hydrocarbon solubilites in sea water at two phase hydrate-water equilibrium for pure methane and for the hydrocarbon mixture were calculated.

This work provides above information at conditions that attempt to simulate the natural behavior of hydrates. 


\subsection{Theoretical Background}

\subsection{Molecular Dynamic Simulations}

A statistical sampling of microscopic or molecular properties (e.g., cavities and their filling by gas molecules) enables the prediction of properties which are macroscopic or measurable with normal tools such as pressure gauges and thermocouples ${ }^{19}$. For the first three-quarters of the last century, statistical thermodynamics was the only bridge available between the molecular and the macroscopic domains ${ }^{20}$. However, the availability of large, fast digital computers has enabled the use of computer simulations into this area.

Computer simulations serve as a means to understand the properties of a group of molecules in terms of their structure and the behavior of molecules at different conditions of the simulations ${ }^{86}$. They can be used to test both theoretical models and the experimental results so as to bring a comparison between them. It has been said by Allen et al. ${ }^{87}$ that "Computer simulations can act as a bridge between the microscopic length and time scales and the macroscopic world of the laboratory". Experiments cannot give an insight into many processes which require a microscopic level of understanding. They also cannot be used for abnormal conditions like very high temperatures or pressures which are not easy to handle in a realistic system. Simulations help us know the feasibility and practicality of such processes. They can complement the experimental work and help us understand the phenomena involved under microscopic level, which cannot be found in other ways. For example, phenomena like the memory effect that has been discussed previously can be better observed and understood through computer simulations than any other methods. Two important simulation techniques commonly used are molecular dynamics and Monte Carlo simulations. The advantage of molecular dynamics over Monte Carlo simulations is that it 
gives a route to determining the dynamical properties of the system like the time dependent responses to perturbations ${ }^{87}$.

Molecular dynamics is a convenient tool which helps simulate a number of molecules covering time periods up to a nanosecond, clearly within the length and the timescales necessary to observe important phenomena pertaining to the hydrate like nucleation, growth and dissociation. They are initiated with a starting configuration and a guess of the interactions between the molecules to determine the properties of the assembly of molecules. If the starting configuration is far from equilibrium, the forces are excessively large resulting in a failure of the simulation in which case an energy minimization is required.

The molecular dynamic technique has been validated for water structures through comparison of calculated properties with experimental thermodynamic data for water, such as the density maximum and the high heat capacity ${ }^{87}$. Since the first applications of molecular simulations to hydrates by Tester et al. ${ }^{88}$ in 1972 , the tool has been widely used to analyze the physical behavior of the hydrates. Molecular simulations have been successfully used in many hydrate research areas such as stability, nucleation, kinetic inhibitors, interfacial properties, spectral properties and other anomalous properties like thermal expansion and thermal conductivity ${ }^{87}$.

MD simulations solve Newton's equation of motion for a system of $\mathrm{N}$ interacting atoms while the forces $F_{i}$ are the negative derivatives of the potential functions $V\left(r_{1}, r_{2}, \ldots, r_{N}\right){ }^{89}$

$$
\begin{gathered}
m_{i} \frac{\partial^{2} r_{i}}{\partial t^{2}}=F_{i}, \quad i=1 \ldots N \\
F_{i}=-\frac{\partial V}{\partial r_{i}}
\end{gathered}
$$

The equations are solved simultaneously in small time steps. The temperature and pressure of the system are kept constant and the coordinates of the atoms are written to an output file at regular intervals. The coordinates as a function of time represent the trajectory of the system. After the 
system reaches equilibrium, many macroscopic properties can be extracted from the output file of the trajectory. The integration of forces between all molecules over several thousand time-steps produces particle trajectories from which time-averaged macroscopic properties can be computed. In a simulation work related to gas hydrates, many properties related to hydrate crystal can be evaluated like the dimensions of the simulation box, pressure, density, temperature, energy, viscosity, bond angles etc. The change in these properties with respect to time studied up to a nanoscale will help in getting a better understanding of the behavior of the hydrate under various conditions. For example a molecular density plot of the simulation cell across the box obtained as a result of these simulations gives an indication of the number of gas molecules moving out of the hydrate phase and also the rate at which they are moving out of the hydrate phase. In molecular dynamics, the simulation is limited by the computer storage and speed. So, the study of long-lived phenomena like nucleation of a hydrate requires a computer with higher storage capacity and speed. Dissolution process is also a slow process compared to dissociation and so requires a computer with higher storage and speed.

However, there are certain limitations of MD simulations and it will therefore be necessary to perform certain checks on the experimental properties to assess the accuracy of the simulation ${ }^{89}$. The approximations are

1. The simulations use classical mechanics to describe the motion of atoms but do not consider the quantum mechanical dynamics. Classical mechanics are not applicable for many hydrogen bonded motions and the bond angle vibrations, for which the bonds are treated as constraints in the equation of motion.

2. Electronic motions are not considered in these simulations and it is assumed that the electrons are in the ground state. 
3. In this work, different force fields were used for each type of molecule obtained by changing the Lennard-Jones parameters. The force fields are also pair-additive which means that all the non bonded interactions result from the sum of non-bonded pair interactions.

4. Periodic boundary conditions are used to avoid real phase boundaries and are more convenient simulating bulk systems compared to smaller systems. When surface effects are not of particular interest, periodic boundary conditions need to be used. It can be applied by creating infinite lattice box, which is a replicate of the basic simulation box considered throughout the space. So, in the course of the simulation, if an atom leaves the basic simulation box, attention can be switched to its incoming periodic image in the neighboring box as shown in the Figure 2.1. Periodic boundary conditions have been used in this work, because we are dealing with bulk phases.

5. Long range interactions are cut-off to avoid expensive calculations and the choice of this cut-off radius depends on the lattice parameter and cannot exceed half the shortest box vector. According to the minimum image convention, each individual particle in the simulation box would interact with particles within this radius around it, which could be either the particles within the simulation box or their closest periodic images. This is shown in the Figure 2.1 by a dotted circle around one of the particles in the central simulation box. 


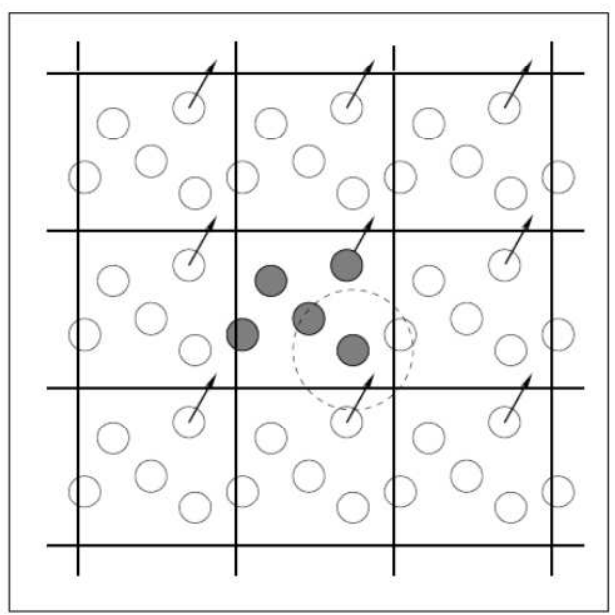

Figure 2.1 Periodic boundary conditions ${ }^{87}$. This figure is taken from Allen, Comp. S. Matter, NIC series, 2004, 23,1

\subsubsection{Energy Minimization}

A molecular dynamic simulation can fail if the starting configuration of the system is far away from the equilibrium causing the forces to be very high. Even in this work, this was observed frequently when it is required to simulate a cell which has two different phases of molecules joined or when some new molecules are required to be inserted in to a group of molecules. This is when energy minimization of the system is necessary. It also helps in removing vacuum if created at the hydrate-water interface during the concatenation of the simulation boxes of hydrate and water phases readjusting the positions of the molecules in the cell. The forces among the molecules depend on the energy function of the system while a potential energy function of a molecular system is a very complex landscape in a number of dimensions ${ }^{89}$. It has one deepest point, the global minimum and very large number of local minima, where all the derivatives of the potential energy function with respect to the coordinates are zero and the second order derivatives are nonnegative. The matrix of second order derivatives, which is called the Hessian matrix, has nonnegative eigen values. 
Knowledge of all local minima, the global minimum and all the saddle points in between the local minima helps in describing the relevant structures and conformations and their free energies, as well as the dynamics of the structural transitions ${ }^{89}$. There is no minimization method that guarantees the determination of a global minimum in a practical amount of time. However, given a starting configuration, it is possible to find the nearest local minimum. The method that is considered is required to search and reach the minimum by systematically moving down the steepest local gradient. The best search method is that which allows excursions into the fourdimensional space. The most popular methods known for the energy minimization are steepest descent method and conjugate gradient method ${ }^{89}$. The steepest descent method takes a step in the direction of the negative gradient irrespective of its history in the previous steps and so gets closer to the nearest minimum very quickly. The conjugate gradient method uses the gradient information of the previous steps and therefore gets much closer to the nearest minimum. Energy minimization for all the simulations in this work was done using the steepest descent method.

\subsubsection{Interaction Functions and Force Field}

A force field is built up from a set of equations called potential functions which are used to generate the potential energies and their derivatives, the forces ${ }^{89}$. Within one set of equations various sets of parameters can be used which are user modified. These parameters vary based on the type of molecule and determine interactions between the molecules. Four types of molecules will be used in this work namely methane, ethane, propane and water. The behavior of the molecules and so some of their properties depend on the force field used for each type of molecule. These potential functions can be subdivided in to three parts ${ }^{90}$.

1. Non-bonded interactions are computed on the basis of a neighbor list, a list of non bonded atoms within a certain radius. The non- bonded interactions contain a repulsion term, a 
dispersion term and a Coulomb term. The Lennard Jones potential between two atoms is given by

$$
V_{L J}\left(r_{i j}\right)=\frac{C_{i j}^{(12)}}{r_{i j}^{12}}-\frac{C_{i j}^{(6)}}{r_{i j}^{6}}
$$

where the parameters $C_{i j}^{(12)}$ and $C_{i j}^{(6)}$ depend on pairs of atom types.

The Colulomb interaction between the charge particles is given by

$$
V_{c}\left(r_{i j}\right)=f \frac{q_{i} q_{j}}{\varepsilon_{r} r_{i j}} ; \quad f=\frac{1}{4 \pi \varepsilon_{0}}
$$

where $\varepsilon_{r}$ is the relative dielectric constant, $q_{i}, q_{j}$ are the electrostatic charge of atoms.

The Lennard-Jones potential is the commonly used potential which includes the repulsion and the dispersion terms while appropriate Coulomb potentials are added if electrostatic charges are present.

2. Bonded interactions are based on a fixed list of atoms. They include bond stretching (2body), bond angle (3-body), and dihedral angle (4-body) interactions. A special type of dihedral interaction called improper dihedral is used to force atoms to remain in a plane or to prevent transition in to its mirror image.

3. Restraints are potentials used for imposing restraints on the motion of the system, either to avoid disastrous deviations, or to include information obtained from the experimental data. They usually include position restraints, angle restraints, distance restraints, orientation and dihedral restraints, all based on fixed lists.

\subsubsection{Lattice Structure used in this Study}

During the 1960s extensive series of crystallographic studies were performed on sI and sII hydrates by Jeffrey and coworkers ${ }^{21}$. Diverse physical techniques were used to study the hydrate 
structure. At first, XRD (single crystal and powder) was used, followed by dielectric techniques and NMR spectroscopy. Applying Raman spectroscopy and single crystal X-ray diffraction for composition and guest distribution of clathrate hydrate emerged in the last decade. In this work, the

host lattice fractional positional parameters reported by McMullan and Jeffery ${ }^{21}$ were selected to represent the oxygen positions within structure I and for structure II by Mark and McMullan ${ }^{20}$.

\subsubsection{Proton Placement}

The water proton distribution that forms the clathrates must be known to understand the configurational characteristics of guest-host interactions inside the cavities. Unfortunately, it is very difficult to measure the proton positions from the conventional diffraction studies. An algorithm was developed by the Sparks ${ }^{80}$ to randomly assign the proton to their respective positions with conforming to Bernal-Fowler Rules ${ }^{25}$ and the constraint that the net dipole of the whole clathrates hydrate structure system should be zero. Nearly, half a million configurations were generated for each clathrate structure and desired water molecule geometry and the resulting configuration with the lowest net dipole moment was then selected as a valid proton assignment. The Bernal-Fowler Rules further refined by Rahman and Stillinger ${ }^{26}$ are outlined below:

1) Water clathrate host lattice consists of intact (non-dissociated) water molecules.

2) The oxygens form the host lattice with very nearly tetrahedral coordination.

3) Each hydrogen bond between two neighboring oxygens is made up of a single proton covalently bonded to one of the oxygens and hydrogen bonded to the other.

4) All proton configurations satisfying above three conditions are equally probable. 


\subsection{Thermodynamic Model}

The dissolution behavior of any gas into or out of a bubble is given as

$$
\text { Mass flux }=A k \Delta C
$$

where, $k$ is the mass transfer coefficient, $A$ is the surface area of the bubble, $\Delta C$ is the concentration difference between the equilibrium solubility of gas and its aqueous concentration. $\Delta C=C_{s}-C_{\alpha}$, is the driving force for the dissolution of the bubble. $C_{s}$ is the interfacial concentration, which is the solubility of a gas at a pressure and temperature and $C_{\propto}$ is the ambient concentration of a gas. The solubility, $C_{S}$, varies depends upon whether hydrate is present or not ${ }^{33,85}$.

The aqueous hydrocarbon solubilities are calculated from the thermodynamic equilibrium. In the V-L equilibrium the hydrocarbon solubilites in the aqueous phase depends on the balance between the chemical potential of the hydrocarbon in the vapor phase $\left(\mu^{v}\right)$ and that in the liquid phase $\left(\mu^{L}\right)$. In the case of hydrates, the solubility of hydrocarbon in aqueous phase depends on the balance between the chemical potential of the hydrocarbon in the hydrate phase $\left(\mu^{H}\right)$ and to that of in the liquid phase $\left(\mu^{L}\right)$. There is no vapor phase present and these hydrates are formed from the dissolved hydrocarbon at the two phase hydrate -liquid water equilibrium $\left(\mathrm{H}-\mathrm{L}_{\mathrm{w}}\right)$.

\subsubsection{VLE modeling}

At thermodynamic equilibrium, the fugacity values are equal in both vapor and liquid phases.

$$
\begin{aligned}
f_{i}^{V}(T, P) & =f_{i}^{L}(T, P) \\
\emptyset_{i}{ }^{V} y_{i} P & =\gamma_{i} x_{i} f_{i}{ }^{0 L}
\end{aligned}
$$

The fugacity coefficient $\left(\emptyset_{i}\right)$ in the vapor phase is calculated using any equation of state (EOS). $y_{i}$ is the vapor phase composition. In the case of gas solubility in the aqueous phase, Henry's law approach is used for each component. The fugacity in the liquid phase for the solute is given as 


$$
f_{i}^{L}(T, P)=H_{i}^{L}(T) x_{i} \exp \left(\left(\frac{v_{i}^{\infty}(T)}{R T}\left(P-P_{i}^{\text {sat }}\right)\right)\right.
$$

$H_{i}{ }^{L}$ is the Henry's constant, $v_{i}{ }^{\infty}$ is the partial molar volume of the solute at infinite dilution. $x_{i}$ is the hydrocarbon composition in the aqueous phase

\subsubsection{Gas hydrate phase equilibrium}

The criterion for the phase equilibrium is the equality of chemical potentials $(\mu)$ of each component in the coexisting phases. At equilibrium,

$$
\mu_{w}{ }^{H}(T, P)=\mu_{w}{ }^{L, \alpha}(T, P)
$$

where $\mu_{w}{ }^{H}(T, P)$ is the chemical potential of water in the hydrate phase, and $\mu_{w}{ }^{L, \alpha}(T, P)$ is the chemical potential of water in the water rich $(L)$ or ice phase $(\alpha)$ at temperature, $T$, and pressure, $P$. The water rich liquid or ice phase is dependent on whether the temperature is above $273.15 \mathrm{~K}$ or not. Using $\mu_{w}{ }^{\beta}$, the chemical potential of hypothetical empty hydrate lattice, the condition for equilibrium can be written as in Equation 2.2.

$$
\begin{aligned}
\Delta \mu_{w}{ }^{\beta-H} & =\Delta \mu_{w}{ }^{\beta-L, \alpha} \\
& \text { where } \quad \mu_{w}{ }^{\beta-H} \equiv \mu_{\beta}{ }^{\beta}-\mu_{w}{ }^{H} \\
\Delta \mu_{w}{ }^{\beta-L, \alpha} & \equiv \mu_{w}{ }^{\beta}-\mu_{w}{ }^{L, \alpha}
\end{aligned}
$$

The initial statistical thermodynamics model to determine the gas hydrates properties was suggested by Barrer and Straut ${ }^{91}$. With the knowledge of the crystal structures of hydrates, van der Waals and Platteeuw ${ }^{92}$ proposed a basic model based on classical statistical thermodynamics corresponding to the three dimensional generalization of ideal localized adsorption, derived the grand canonical partition function for water, with the following assumptions.

1) Each cavity can contain at most one gas molecule. 
2) The interaction between a gas and water molecule can be described by a pair potential functions, and the cavity can be treated as perfectly spherical.

3) The free energy contribution of the water molecules is independent of the mode of dissolved gases (cage distortions are neglected)

4) There is no interaction between the gas molecules in different cavities and the guest molecule interacts only with the nearest neighbor water molecules (guest-guest interactions are neglected).

The chemical potential difference between the empty lattice and fully filled hydrate lattice can be expressed as

$$
\Delta \mu_{w}^{\beta-H}=-R T \sum_{i=1}^{n} v_{i} \ln \left(1-\sum_{j} \theta_{j i}\right)
$$

where $v_{i}$ is the number of $i$-types cavities per water molecule, $R$ is the gas constant and $T$ is the temperature. $\theta_{j i}$ is the fractional occupancy of $i$-type cavities with $j$-type guest molecules. $n$ is the number of cavities and is equal to 2 for sI and sII, $n=3$ for structure H. From the Equation 2.3, the chemical potential of the hydrate is reduced by the potential interactions of the guest and the host water molecules. The greater the fraction of cavities occupied lesser is the chemical potential of the hydrate and water. Clathrate hydrates are non stoichiometric compounds, therefore the cage occupancy, $\theta_{j i}$, is $\leq 1$ and also a function of equilibrium conditions. Mathematically, the cage occupancy, $\theta_{j i}$, follows the Langmuir isotherm and expressed in terms of Langmuir constant as

$$
\theta_{j i}=\frac{C_{j i} f_{j}}{1+\sum_{i} C_{j i} f_{j}}
$$

where $f_{i}$ is the fugacity of hydrate forming species $i$ in the phase at which the hydrate phase is in equilibrium. $C_{j i}$ is the temperature-dependent Langmuir constant for species $i$ in cavity $j$ defined as 


$$
C_{j i}=\frac{Z_{j i}}{k T}=\frac{1}{8 \pi^{2} k T} \int_{V} \exp \left(-\frac{\Phi(r, \theta, \phi, \alpha, \beta, \gamma)}{k T}\right) r^{2} \sin \theta d r d \theta d \phi d \alpha \sin \beta d \beta d \gamma
$$

where $Z_{j i}$ is the configurational integral, which depends on the total interaction potential $\Phi=\sum \Phi_{\mathrm{ij}}$ between the guest molecule and the host molecules surrounding it ${ }^{80,93} . Z_{j i}$ is a function of the polar coordinates $r, \theta, \phi$ of the guest molecule and $\alpha, \beta, \gamma$, the Euler angles that describes the orientation of guest molecule. The Langmuir constant is actually the description of the affinity of the empty cavity for a molecule to occupy this cavity, higher values of the Langmuir constant indicate that a guest molecule is more likely to be encaged. Langmuir constant will approach to zero when the guest molecule is small compared to the cavity.

For the structure I hydrate, the unit cell has 46 water molecules with 2 small cavities and 6 large cavities. The number of small cavities per water molecule, $v_{1}$, is equal to $1 / 23$, the number of large cages, $v_{2}$, is equal to $3 / 23$, the complete expression for a pure component structure I water clathrates system is

$$
\frac{\Delta \mu_{w}^{\beta-H}}{k T}=\frac{1}{23} \ln \left(1+C_{j 1} f_{j}\right)+\frac{3}{23} \ln \left(1+C_{j 2} f_{j}\right)
$$

The structure II hydrate unit cell has 136 water molecules with 16 small cavities and 8 large cavities. The ratio of small cavities to water molecules, $v_{1}$, equals $2 / 17$, and the number of large cages, $v_{2}$, is equal to $1 / 17$. The complete expression for a pure component structure II water clathrates system is

$$
\frac{\Delta \mu_{w}^{\beta-H}}{k T}=\frac{2}{17} \ln \left(1+C_{j 1} f_{j}\right)+\frac{1}{17} \ln \left(1+C_{j 2} f_{j}\right)
$$

The chemical potential difference, $\Delta \mu_{w}$, between the hypothetical empty hydrate lattice and water in the hydrate phase is given by Holder et $\mathrm{al}^{4}$ as 


$$
\frac{\Delta \mu_{w}^{\beta-L, \alpha}(T, P)}{k T}=\frac{\Delta \mu_{w}^{\beta-L, \alpha}\left(T_{o}, 0\right)}{k T}-\int_{T_{o}}^{T} \frac{\Delta h_{w}^{\beta-L, \alpha}}{k T^{2}} d T+\int_{o}^{P} \frac{\Delta V_{w}^{\beta-L, \alpha}}{k T} d P-\ln a_{w}
$$

where $\Delta \mu_{w}^{\beta-L, \alpha}\left(T_{o}, 0\right)$ is the reference chemical potential difference at the reference temperature, $T_{o}$, and zero pressure. The reference temperature, $T_{o}$, is the ice point temperature. In case of methane hydrate the ice point temperature $T_{o}=272.95 \mathrm{~K}$ and in case of carbon dioxide hydrate, $T_{o}$ is $271.75 \mathrm{~K}$. The depression in the ice point temperature for $\mathrm{CO}_{2}$ hydrate is due to the high solubility of carbon dioxide in water. The second term on the left of Equation 2.16 gives the temperature dependence at constant pressure. The third term corrects the pressure to the final equilibrium pressure and the last term corrects the chemical potential from pure water phase to water rich solution. The temperature dependent enthalpy difference is given by Equation 2.17.

$$
\Delta h_{w}^{\beta-L, \alpha}=\Delta h_{w}^{o}\left(T_{o}\right)+\int_{T_{o}}^{T} \Delta C_{P} d T
$$

where, $\Delta h_{w}{ }^{o}\left(T_{o}\right)$ is the reference enthalpy difference between the empty hydrate lattice and the pure water phase at reference temperature $T_{o}$. The heat capacity difference between the empty hydrate lattice and the pure water phase, $\Delta C_{P}$, is also temperature dependent and it is approximated by the following expression

$$
\Delta C_{P}=\Delta C_{P}^{\mathrm{o}}\left(T_{o}\right)+b\left(T-T_{o}\right)
$$

where $\Delta C_{P}{ }^{\circ}\left(T_{o}\right)$ is the reference heat capacity difference at the reference temperature $T_{o}$. The constant, $b$, represents the dependence of heat capacity on the temperature. Two different expressions must be used for the water in liquid phase and in solid phase. The volume difference, $\Delta V_{w}{ }^{\beta-L, \alpha}$, is assumed to be constant. The last term in the Equation 2.16 is activity of water, $a_{w}$, is defined as 


$$
a_{w}=\frac{f_{w}{ }^{L}}{f_{w}}
$$

where $f_{w}{ }^{L}$ is the fugacity of water in the water rich aqueous phase and $f_{w}$ is the water fugacity at the reference state, the pure water phase. The thermodynamic reference properties used in this work are given in Table 2.1.

Table 2.1 Heat capacity and volumetric reference properties between the empty hydrate lattice and fluid phase (liquid water or ice)

\begin{tabular}{|c|c|c|}
\hline Constants & Structure II & Reference \\
\hline$\Delta \mathrm{V}_{w}^{\beta-\alpha}\left(\mathrm{m}^{3} / \mathrm{mol}\right)$ & $3.0 \times 10^{-6}$ & 94 \\
\hline$\Delta \mathrm{V}_{w}{ }^{L-\alpha}\left(\mathrm{m}^{3} / \mathrm{mol}\right)$ & $-1.598 \times 10^{-6}$ & 95 \\
\hline$\Delta \mathrm{H}_{w}{ }^{L-\alpha}(\mathrm{J} / \mathrm{mol})$ & 6009.5 & \\
\hline$\Delta \mathrm{C}_{p}^{\beta-\alpha}\left(T_{0}\right)$ & 0.565 & \\
\hline$b^{\beta-\alpha}$ & 0.002 & 95 \\
\hline$\Delta \mathrm{C}_{p}^{\beta-L}\left(T_{0}\right)$ & -37.32 & \\
\hline$b^{\beta-L}$ & 0.179 & \\
\hline
\end{tabular}

\subsubsection{Langmuir constant and reference parameters}

The most important term in the van der Waals and platteeuw ${ }^{92}$ model is the Langmuir constant. The Langmuir constant depends on the pairwaise additive of the guest-host interactions potentials. For a guest molecule $j$ in a cavity of type $i, C_{J i}$, is directly related to the six dimensional configurational integral over a system volume $V$ defined by

$$
Z_{j i}=\frac{1}{8 \pi^{2}} \int_{V} \exp \left(-\frac{\Phi(r, \theta, \phi, \alpha, \beta, \gamma)}{k T}\right) r^{2} \sin \theta d r d \theta d \phi d \alpha d \beta d \gamma
$$


where $Z_{j i}$ is the configurational integral, which depends on the interaction potential, $\Phi$, between the guest molecule $j$ in the cavity $i$ and all the host molecules surrounding it. The interaction potential is a function of the position and orientation of the guest in the cavity and is given by the spherical coordinate's $r, \theta, \phi$ and the Euler angles $\alpha, \beta$ and $\gamma$ which describe the orientation of the guest. The factor of $8 \pi^{2}$ is the normalizing constant coming from the volumetric integration. The total interaction potential $\Phi=\sum_{\mathrm{ij}} \Phi_{\mathrm{ij}}$ between the guest and all the host water molecules must be represented properly to calculate the configurational integral accurately. The original work by van der Waals and Platteuw used the Lennard Jones (L-J) 6-12 pair potential. McKoy and Sinangolu ${ }^{16}$ suggested that the Kihara potential is better than the Lennard Jones potential. The potential parameters were obtained by empirically fitting to the experimental hydrate dissociation data. However, these empirically-fitted potential parameters are aphysical and don't match those determined using gas phase experimental data ${ }^{94,96-97}$.

Physically based intermolecular potentials are needed to more accurately calculate the Langmuir constant. Work by Klauda and Sandler ${ }^{98}$, Cao et al. ${ }^{81}$, Anderson et al. ${ }^{93}$ and Velaga and Anderson ${ }^{99-100}$ have shown that ab initio quantum mechanical calculations provides an independent method to develop the guest-host intermolecular potentials. Also, a full configurational integral has to be evaluated when calculating the Langmuir constant instead of using the Lennard-Jones Devonshire (LJD) spherical cell approximation ${ }^{80-81,101}$. The LJD spherical cell approach simplifies the multidimensional integral to a one-dimensional integra ${ }^{102}$. The results obtained with these intermolecular potentials have superior results in terms of hydrate phase equilibrium and cage occupancies.

Anderson et al. $^{93}$ calculated the Langmuir constants from the site-site $a b$ initio intermolecular potentials by calculating the full six dimensional integral for methane gas hydrates. 
The resulting Langmuir constants computed from the $a b$ initio data are fitted to the van't off temperature dependence functional form ${ }^{78}$, given by Equation 2.13 .

$$
C_{j i}(\beta)=C_{0 j i} \exp \left(m_{j i} \beta\right)
$$

where, $\beta=1 / k T, C_{0 j i}$ and $m_{j i}$ are specific to the guest molecule $i$, and the cavity $j$ occupied. $C_{0 j i}$ and $m_{j i}$ are cell potential parameters and the method above is called Cell Potential method ${ }^{78,97,99}$.

Holder et al. ${ }^{103}$ suggested that the reference chemical potential difference, $\Delta \mu_{w}{ }^{0}$, varies with the size of the guest molecule instead of using a single value for all the guest molecules. There will be a distortion in the lattice of the hydrate when the size of the guest molecule is increased. Pradhan ${ }^{104}$ found that the reference chemical potential difference value increases with the increase in size of the guest molecule: by fitting the experimental data while slightly adjusting the Kihara parameters for some guest molecules. The molecular diameter of methane is $4.36 \AA$, ethane is $5.5 \AA$ and propane is $6.28 \AA$. The reference parameters $\Delta \mu_{w}{ }^{0}$ and $\Delta H_{w}{ }^{0}$ for ethane, methane and propane have to use different values in order to calculate accurate phase equilibrium and cage occupancies. 


\subsection{Molecular Dynamics Simulations to Study the Dissolution of Structure II Hydrates Containing Methane/Ethane/Propane Mixture}

\subsection{Introduction}

Natural gas hydrates are present in the permafrost and in the ocean sediments and are considered as a potential future energy source because these naturally occurring hydrates comprise one of the largest reservoir of methane on earth ${ }^{19}$. The favorable conditions to form hydrates are low temperatures and high pressures. Hydrates once formed can be destabilized when there is increase in temperature or decrease in pressure moving out of the hydrate stability zone. This process is called hydrate dissociation and in this process hydrate dissociates in to liquid water and hydrate forming gas. Hydrates also dissolve over time if the surrounding fluid is under-saturated in the hydrate forming gas even though the hydrates exist in the hydrate stability zone. In addition to temperature and pressure, the chemical potential of the gas in the hydrate must be equal to the surrounding water to be in thermodynamic equilibrium. If the chemical potential is in disequilibrium the hydrate forming gas will dissolve into the surrounding water in a process called dissolution, in this process hydrate decomposes in to liquid water and aqueous gas. Most of the hydrate deposits in the oceans are typically located beneath the oceanic sediments. However, there are some hydrate deposits which were found to be exposed to seafloor; these outcrop hydrates are most vulnerable to dissolution. Outcrops of hydrates have been found in the Gulf of Mexico, the Atlantic and Pacific Oceans ${ }^{31,105}$ in the United States waters.

Much work was done over the years to understand the thermodynamics and kinetics of the hydrate dissociation $^{19}$, but hydrate dissolution is not a well understood phenomena. Recently, hydrate dissolution has become an active area of interest $18,30,41,43,45,50$. The theoretical predictions and the dissolution studies on the artificial methane hydrates (formed sI hydrates) suggest that the 
methane hydrate dissolution is a diffusion controlled process ${ }^{30,44-45}$. The methane concentration in the seawater surrounding the outcrop hydrate sites are well below saturation ${ }^{106}$, so the naturallyoccurring outcrop hydrates will dissolve over time. However, contrary to these theoretical predictions, studies on the natural gas hydrates on the sea floor sites at Gulf of Mexico ${ }^{107}$, the northen Cascadia Margin ${ }^{43}$ and in Santa Monica $\operatorname{Basin}^{108}$ show little or no change in the morphology of these outcrop hydrates over years time ${ }^{43}$. Egorov et al.$^{50}$ suggested that the exposed hydrates remain stable by reforming within the sediments at the same rate as they dissolve at the exposed surface, with the so called "push-up-pop" model. If this is true, then the sediments surrounding the surface hydrate should have near saturation concentration of hydrate forming gas ${ }^{43}$. But the observations by Lapham et al. ${ }^{43}$ on the outcrop natural gas hydrates at northern Cascadia Margin and at Gulf of Mexico suggest that the in situ methane concentrations in the sediments surrounding the gas hydrates are greatly under-saturated with respect to the expected values of equilibrium with methane gas hydrates.

Dissolution rates measured by Hester et al. ${ }^{41}$ of exposed naturally formed hydrates at Barkley Canyon, Cascadia Margin are $117 \mathrm{~cm} /$ year for white hydrate and $60 \mathrm{~cm} /$ year for yellow hydrates. The composition of the white hydrate and yellow hydrates are different, with yellow hydrates having lower concentrations of methane content than the white hydrate, i.e. there is more amount of ethane and propane content in yellow hydrate than in white hydrate. They found that the dissolution of white hydrate is faster than the yellow hydrate. Rehder et al ${ }^{30}$ synthesized pure methane hydrates in the laboratory and then they were brought to the Barkley Canyon hydrate site to measure the dissolution rate. They measured dissolution rates of $150 \mathrm{~cm} / y e a r$. The experimental study of Rehder et al. ${ }^{30}$ and Bigalke et al. ${ }^{109}$ were done on pure methane hydrates which forms sI hydrate under experimental conditions. The dissolution rates measured by Herster et al. ${ }^{41}$ is for 
thermogenic hydrates, which likely forms sII hydrates because of the presence of higher hydrocarbons.

Naturally-occurring hydrates in the oceanic environment are generally a sII mixed hydrate because of the presence of higher hydrocarbons like ethane and propane. The slower dissolution

rates observed by Hester al. ${ }^{41}$ to that of other studies (sI hydrates) might be because of the sII hydrates. Thermogenic hydrates contain ethane and propane and these higher hydrocarbons might be responsible for the lower dissolution rates. Even in the Hester et al. ${ }^{41}$ observations for white hydrates and yellow hydrates, the yellow hydrate has higher amount of ethane and propane composition to that of white hydrate and the lower dissolution of yellow hydrate to that of white hydrate can be due to the presence of higher hydrocarbons.

To explain the different dissolution rates, we hypothesized that the presence of higher hydrocarbons like ethane and propane which forms sII hydrate enhances the stability of the hydrates. The main objective of this chapter is to study the effect of propane and ethane on the stability of the sII mixed hydrate using molecular dynamics (MD) simulations.

\subsection{Molecular Dynamic (MD) Simulation}

Molecular simulations have been proven to be a powerful tool to elucidate various properties of hydrate. Several MD simulations reported over the years exploring the thermophysical and dynamical properties of hydrates ${ }^{110-111}$, formation $^{112}$ and dissociation ${ }^{53,60,113-115}$ of gas hydrates, inhibition mechanism and effect of inhibitors on the hydrate stability ${ }^{116}$ at the molecular scale. Most of the MD simulations reported exploring the hydrate properties are for methane hydrate. MD simulations were used to study the dissociation behavior of hydrates, understanding the effect of 
cage occupancy and temperature on the dissociation of hydrate ${ }^{53,60,113-114}$. Though many issues were still remaining to be resolved, they were at least able to demonstrate the dissociation behavior.

In this work we have used MD simulations to study the dissolution behavior of sII mixed hydrate. The simulations were carried out with different large cage occupancy obtained by changing methane, ethane and propane occupancy.

\subsubsection{Force field and initial conditions}

All MD simulations in this work were done in the isothermal-isobaric (NPT) ensemble. In this study, the TIP4P/ice force field was used for water ${ }^{117}$. Studies by Vega and co-workers ${ }^{118-120}$ have shown that TIP4P/ice model gives the accurate predictions for the melting point of ice and also methane hydrate dissociation pressure compare to other water models. The TIP4P/ice model has an additional interacting site "M" located on the bisector of the H-O-H angle $0.1577 \AA$ away from the oxygen atom towards the hydrogen atom. The TIP4p/ice water model is given in Figure 3.1. The force field for propane and ethane is represented by OPLS model ${ }^{121}$ and for methane by Anderson et al. ${ }^{93,116}$ model. The potential parameters of the force field are given in Table 3.1. The Lorentz-Berthelot combination $^{122}$ rules were used to calculate the Lennard-Jones parameters between the water and guest molecules and are given by

$$
\begin{gathered}
\sigma_{i j}=\frac{\sigma_{i i}+\sigma_{j j}}{2} \\
\varepsilon_{i j}=\sqrt{\varepsilon_{i i} \varepsilon_{i i}}
\end{gathered}
$$

Periodic boundary conditions were used in all directions for all the simulations. The cutoff radius for the $\mathrm{LJ}$ interactions was chosen to be $9 \AA$ for all the systems. Ewald summation ${ }^{123}$ was used to account for the long range electrostatic interactions. The Nose-Hoover thermostat for NPT simulations and berendsen thermostat coupling scheme for canonical ensemble (NVT) simulations 
were used for the temperature coupling of the system. The system was coupled to a Berendsen pressure coupling for controlling the pressure. The GROMACS ${ }^{89}$ package was used to perform all the MD simulations.

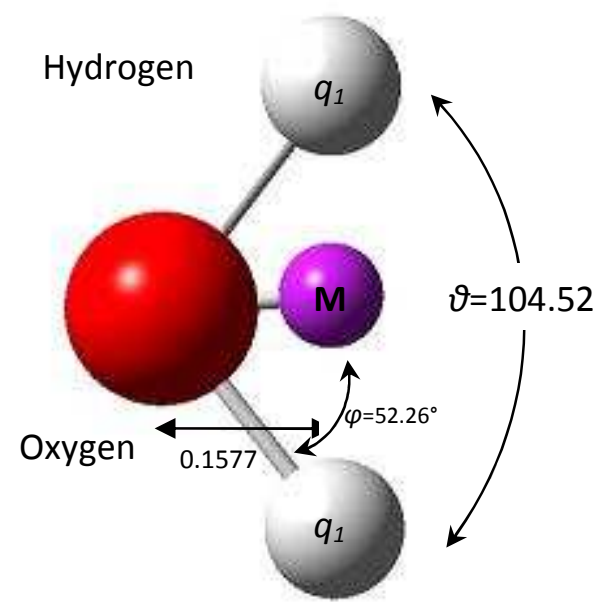

Figure 3.1 TIP4P-ice water model ${ }^{117}$

Table 3.1. Intermolecular potential parameters for methane, ethane, propane and water. Atoms marked in bold indicate interacting site.

\begin{tabular}{|c|c|c|c|c|}
\hline Interaction & $\boldsymbol{\varepsilon}(\mathrm{kcal} / \mathrm{mol})$ & $\boldsymbol{\sigma}(\AA)$ & $\mathrm{Q}($ charge $)$ & $\mathrm{Q}_{\mathrm{M}}$ \\
\hline \multicolumn{5}{|c|}{ OPLS potential Parameters ${ }^{121}$} \\
\hline $\mathrm{H}_{4} \mathrm{C}$ & 0.066 & 3.5 & -0.24 & \\
\hline $\mathrm{H}_{3} \mathrm{C}$ & 0.066 & 3.5 & -0.18 & \\
\hline $\mathrm{H}_{2} \mathrm{C}$ & 0.066 & 3.5 & -0.12 & \\
\hline $\mathrm{HC}$ & 0.030 & 2.5 & 0.06 & \\
\hline \multicolumn{5}{|c|}{ Anderson et al. model for methane $\mathrm{e}^{93,116}$} \\
\hline $\mathrm{H}_{4} \mathrm{C}$ & 0.18 & 3.5 & -0.24 & \\
\hline $\mathrm{HC}$ & 0.03 & 2.5 & 0.06 & \\
\hline \multicolumn{5}{|c|}{ TIP4P/ice model for water ${ }^{124}$} \\
\hline $\mathrm{H}_{2} \mathrm{O}-\mathrm{OH}_{2}$ & 0.2107 & 3.1668 & - & -1.174 \\
\hline $\mathrm{HOH}$ & - & - & 0.5897 & \\
\hline
\end{tabular}




\subsubsection{Simulation box}

The simulation box was constructed using the programs in the GROMACS package based on the lattice structure of a unit cell of hydrate. A structure II unit cell has 16 small cavities and 8 large cavities with 136 water molecules. The cavity centers of sII hydrate unit cell are given in Table 3.2. The Cartesian coordinates of the hydrate forming gas molecules are given in Table 3.3, Table 3.4 and Table 3.5 for methane, ethane and propane respectively. The guest gases occupy the cavity centers. The initial hydrate structure was generated from the oxygen fractional positional reported by Mark and McMullan for sII hydrate. The hydrogen atoms were assigned to the oxygen atom maintaining the water geometry and the orientations were satisfied following the BernalFowler Rules ${ }^{125}$ and with near zero-net dipole of the whole system. The water molecule positions for sII hydrate unit cell are given in Apendix A. The lattice constant of structure II hydrate is 17.03 $\AA$ A. The unit cell is replicated to get the desired size of the simulation box.

The distribution of the guest molecules in the cages were done based on the thermodynamic results of methane-ethane-propane mixed hydrate which in-turn based on the van der Waals and

Platteeuw thermodynamic model ${ }^{78,126}$. The thermodynamic model provides the cage occupancies in the large and small cages of mixed hydrate. So for a sII hydrate, methane occupies the small cages and large cages, ethane and propane occupies only large cages. In this work for a fully-occupied sII hydrate, a unit cell has $100 \%$ occupancy of methane in small cage, and large cages are $50 \%$ occupied by ethane, $25 \%$ by propane and $25 \%$ by methane. 
Table 3.2 Relative positions of all cavities in a unit cell of sII hydrate showing cavity centers.

\begin{tabular}{|c|c|c|c|c|}
\hline \multicolumn{5}{|c|}{ Cavity centers in unit cell } \\
\hline molecule & Cavity & $x$ & $y$ & $z$ \\
\hline 1 & \multirow{16}{*}{ Small $\left(5^{12}\right)$} & 4.3250 & 4.3250 & 4.3250 \\
\hline 2 & & 0.0000 & 8.6500 & 12.9750 \\
\hline 3 & & 8.6500 & 12.9750 & 0.0000 \\
\hline 4 & & 12.9750 & 0.0000 & 8.6500 \\
\hline 5 & & 4.3250 & 12.9750 & 12.9750 \\
\hline 6 & & 0.0000 & 0.0000 & 4.3250 \\
\hline 7 & & 8.6500 & 4.3250 & 8.6500 \\
\hline 8 & & 12.9750 & 8.6500 & 0.0000 \\
\hline 9 & & 12.9750 & 4.3250 & 12.9750 \\
\hline 10 & & 8.6500 & 8.6500 & 0.0000 \\
\hline 11 & & 0.0000 & 12.9750 & 8.6500 \\
\hline 12 & & 4.3250 & 0.0000 & 0.0000 \\
\hline 13 & & 12.9750 & 12.9750 & 4.3250 \\
\hline 14 & & 8.6500 & 0.0000 & 12.9750 \\
\hline 15 & & 0.0000 & 4.3250 & 0.0000 \\
\hline 16 & & 4.3250 & 8.6500 & 8.6500 \\
\hline 17 & \multirow{8}{*}{ Large $\left(5^{12} 6^{4}\right)$} & 10.8125 & 10.8125 & 10.8125 \\
\hline 18 & & 6.4875 & 15.1375 & 6.4875 \\
\hline 19 & & 10.8125 & 2.1625 & 2.1625 \\
\hline 20 & & 6.4875 & 6.4875 & 15.1375 \\
\hline 21 & & 2.1625 & 10.8125 & 2.1625 \\
\hline 22 & & 15.1375 & 15.1375 & 15.1375 \\
\hline 23 & & 2.1625 & 2.1625 & 10.8125 \\
\hline 24 & & 15.1375 & 6.4875 & 6.4875 \\
\hline
\end{tabular}


Table 3.3 Cartesian coordinates of methane molecule with respect to the central atom carbon

\begin{tabular}{cccc}
\hline atom & $x$ & $y$ & $z$ \\
\hline $\mathrm{C}_{1}$ & 0.0000 & 0.0000 & 0.0000 \\
$\mathrm{H} 1$ & -0.1874 & 0.0417 & -1.0803 \\
$\mathrm{H} 2$ & -0.9464 & -0.1051 & 0.5441 \\
$\mathrm{H} 3$ & 0.6516 & -0.8528 & 0.2276 \\
$\mathrm{H} 4$ & 0.4886 & 0.9257 & 0.3280 \\
\hline
\end{tabular}

Table 3.4 Cartesian coordinates of ethane molecule. The midpoint between the $\mathrm{C} 1$ and $\mathrm{C} 2$ is the center of the cage.

\begin{tabular}{cccc}
\hline atom & $x$ & $y$ & $z$ \\
\hline $1 \mathrm{C}$ & -0.7067 & 0.0116 & -0.2573 \\
$2 \mathrm{C}$ & 0.7067 & -0.0116 & 0.2573 \\
$1 \mathrm{H} 1$ & -0.8873 & 0.8799 & -0.9041 \\
$1 \mathrm{H} 2$ & -0.9379 & -0.8862 & -0.8453 \\
$1 \mathrm{H} 3$ & -1.4353 & 0.0597 & 0.5624 \\
$2 \mathrm{H} 4$ & 0.8885 & -0.8812 & 0.9023 \\
$2 \mathrm{H} 5$ & 1.4351 & -0.0567 & -0.5625 \\
$2 \mathrm{H} 6$ & 0.9368 & 0.8849 & 0.8476 \\
\hline
\end{tabular}


Table 3.5 Cartesian coordinates of propane molecule. The center of the cage is at atom $2 \mathrm{C}$.

\begin{tabular}{cccc}
\hline atom & $x$ & $y$ & $z$ \\
\hline $1 \mathrm{C}$ & 1.3419 & 0.5696 & 0.4019 \\
$2 \mathrm{C}$ & 0.0000 & 0.0000 & 0.0000 \\
3C & -1.0308 & 0.1746 & 1.0925 \\
$1 \mathrm{H} 1$ & 1.7351 & 0.0800 & 1.3026 \\
$1 \mathrm{H} 2$ & 1.2763 & 1.6442 & 0.6184 \\
$1 \mathrm{H} 3$ & 2.0865 & 0.4398 & -0.3937 \\
$2 \mathrm{H} 4$ & -0.3518 & 0.4872 & -0.9307 \\
$2 \mathrm{H} 5$ & 0.1062 & -1.0740 & -0.2496 \\
$3 \mathrm{H} 6$ & -2.0042 & -0.2349 & 0.7939 \\
$3 \mathrm{H} 7$ & -1.1818 & 1.2338 & 1.3400 \\
$3 \mathrm{H} 8$ & -0.7296 & -0.3355 & 2.0172 \\
\hline
\end{tabular}

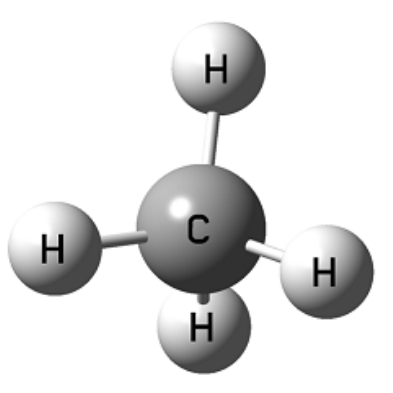

(a)

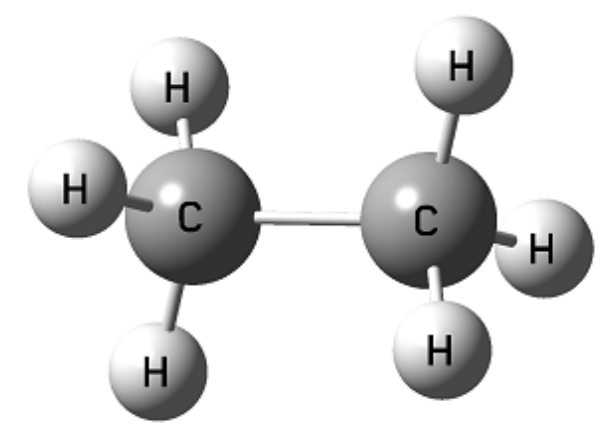

(b)

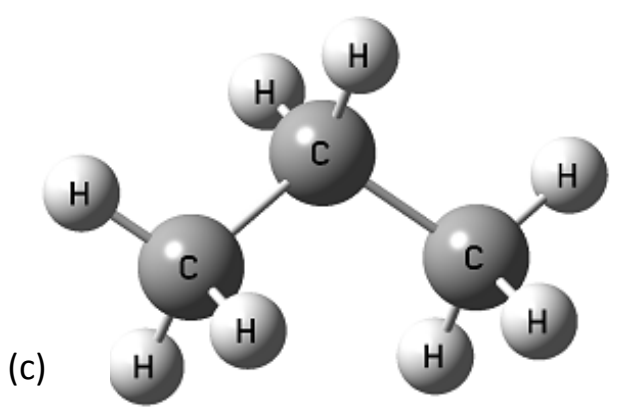

Figure 3.2 Ball and stick model of (a) Methane, (b) Ethane and (c) Propane molecules 


\subsection{Analysis of the Simulation}

Visual molecular dynamics (VMD) was used to visualize the entire simulation which helped to get a clear vision of the hydrate structures and the movement of the molecules in the simulation box. It is required to estimate the rate at which the gas molecules move out of the hydrate phase to the surrounding water as a result of the dissolution process. Analyses of all the simulations were done based on the density plots and energy plots of the simulation box. Plots of the average total energy of the simulation box at different times during the course of simulation were used to help determine whether the simulation box reached equilibrium with respect to the dissolution process.

Number density plots of the simulation cell across the box in the z-direction helped to estimate the number of gas molecules that are present in water and hydrate phases at different stages of the simulation and also observed the movement of the liquid-hydrate interface. The number density profiles was obtained by

$$
\langle\rho(z)\rangle=\frac{\langle N(z)\rangle}{A \Delta z}
$$

where $N(z)$ is the number of molecules in a slab located between $z$ and $\Delta z, A$ is the surface area, $\Delta z$ is the width of the slab. In this work, the simulation cell was divided in to 100 slabs of constant width in the z-direction to generate the density profile of the selected group of molecules across the simulation box. The brackets denote time averaging. Density plots were calculated from the mean of the last $1 \mathrm{~ns}$ simulation.

\subsubsection{Identification of hydrate and liquid phases}

It is important to identify in which phase these gas molecules are present at each stage of a simulation, especially the ones present at the hydrate-water interface. Therefore, a phase recognition code given by Baez and Clancy ${ }^{10}$ was used, in which they employed different criteria required to differentiate the water molecules among three phases, water, ice and hydrate. An 
angular parameter has been defined, which is calculated for each water molecule $i$, where $n_{i}$ is the number of nearest neighbors of molecule $i$.

$$
F_{i}=\sum_{j=1}^{n_{i}-1} \sum_{k=j+1}^{n_{i}}\left(\left|\cos \theta_{j i k}\right| \cos \theta_{j i k}+0.11\right)^{2}
$$

The nearest neighbor molecules of a molecule are considered to be those present within a distance that corresponds to the first minimum in the oxygen-oxygen pair correlation for the ice or hydrate. This distance was taken to be $3.5 \AA . \quad \theta_{j i k}$ is the triplet angle between the $\mathrm{O}$ atoms of molecules $j, i$ and $k$. For a molecule $i$ with $n_{i}$ neighbor molecules, there will be $n_{i}\left(n_{i}-1\right) / 2$ independent triplet angles with $i$ as central molecule. In ice and hydrate, the water molecules are tetrahedrally bonded. Therefore, they have 6 independent angles for each molecule $i$ and the cosine of each angle $\theta_{j i k}$ would be close to -0.33 . So, the value of $F_{i}$ for a particular water molecule gives the degree of deviation from being tetrahedrally bonded to its neighbors. If the value of $F_{i}$ is close to zero $\left(F_{i}<0.4\right)$, it indicates a tetrahedral bonding and the molecule is supposed to be in an ice-like or a hydrate-like region. Otherwise, it implies that the molecule is in water-like region.

The categorization between hydrate-like and ice-like water molecules is then done based on the identification of five-membered rings, observed in hydrates and not in ice. A water molecule in hydrate shares only four, five or six pentamers, unlike that observed in the liquid water. The angular order parameter and the number of five-membered rings in which the water molecule is present, in conjunction set the criteria required to categorize the water molecules among 3 different phases; ice, water and hydrate. Therefore, a water molecule is considered to be ice-like if $F_{i}<0.4$ and no pentamers are identified, hydrate-like if $F_{i}<0.4$ and the molecule is found to form a part of four, five or six pentamers and liquid-like in all the other cases.

A reclassification of these water molecules is done based on the phase identity of its neighbor molecules. If a water molecule has three or more neighbors with different identity, it is 
reassigned to that identity. The hydrate-like water molecules are designated to clusters based on this reclassification. Clusters having less than eight water molecules are reassigned to liquid water. After the classification of all the water molecules, the methane molecules are assigned to each cluster, if there are more than ten hydrate-like water molecules within a distance of $5.5 \AA$. This phase recognition algorithm has been tested on pure hydrate and was found to give correct phase recognition of the molecules in the simulation cell.

\subsection{Validation of Intermolecular potentials}

A $2 \times 2 \times 2$ simulation box was created containing a total of 8 unit cell for sII hydrate. A replica of a $2 \times 2 \times 2$ sII hydrate is shown in Figure 3.3. To test the OPLS and Anderson et al. intermolecular potentials, the lattice constant of sII hydrate was determined by running NPT simulations at a constant pressure and at different temperatures with full occupancy. A time step of $1 \mathrm{fs}$ was used in all the simulations of lattice constant calculations. The simulations were carried out for $800 \mathrm{ps}$.

For each simulation, a plot of box dimensions with respect to time was obtained from which lattice constant was evaluated. The lattice constant was obtained when the system has reached the equilibrium; where there is no further drift in the volume of the simulation box. The lattice constant was evaluated from the average value of the last 200 ps simulation. The plot of lattice constant with time is given in Figure 3.4 for $260 \mathrm{~K}$.

Simulations were done at different temperatures and the lattice constants were calculated. The lattice constant of the simulation cell increases with increase in temperature. Figure 3.5 shows the lattice constant value at different temperatures. The lattice constant simulated at $275 \mathrm{~K}$ was 
found to be $1.732 \mathrm{~nm}$ for the intermolecular potentials used. The experimental lattice constant value at $275 \mathrm{~K}$ is $1.738 \mathrm{~nm}$. This serves as the validation of the intermolecular potentials that were used in the dissolution studies.

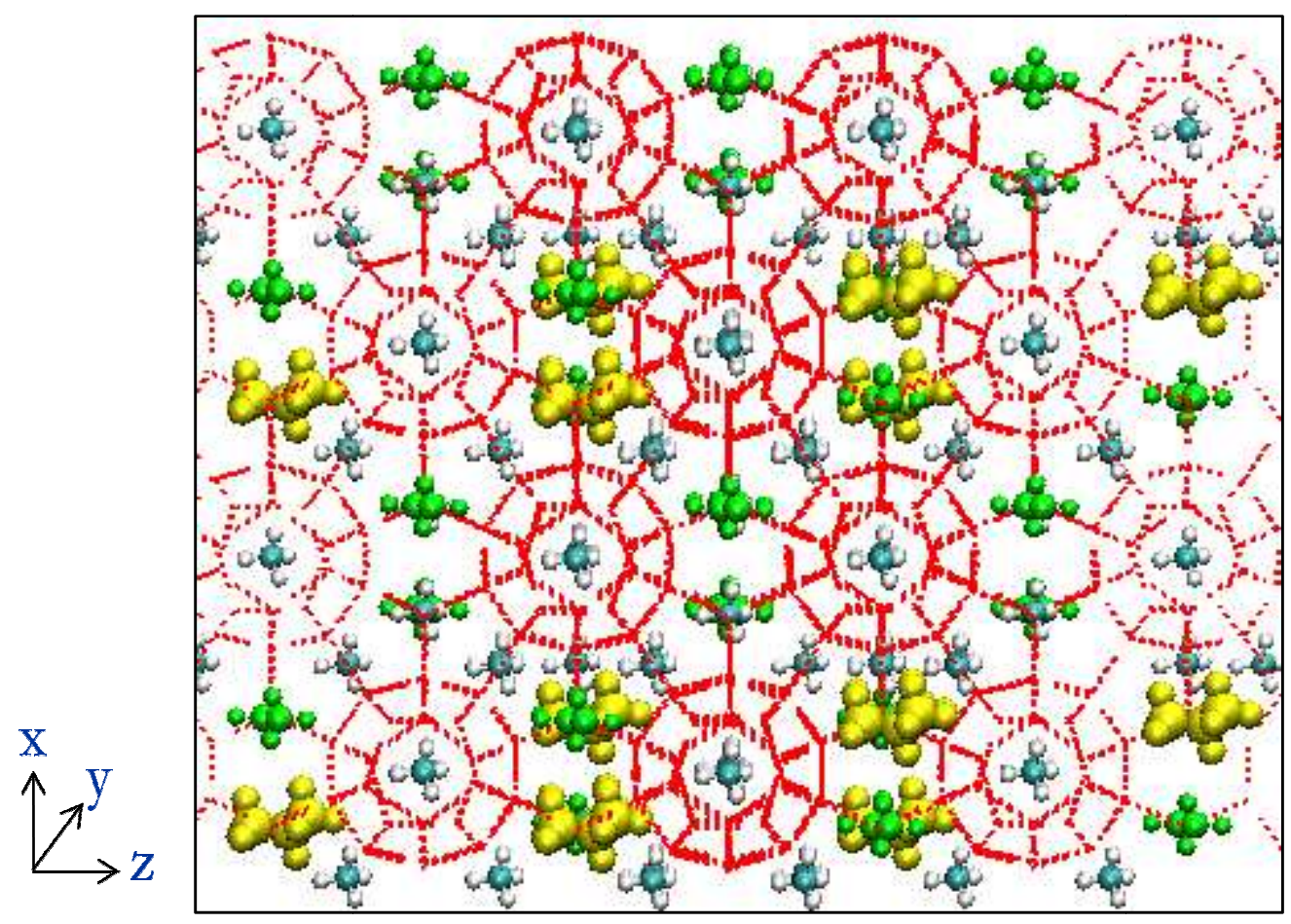

Figure 3.3 A 2-dimensional view of an 8 unit cell $(2 \times 2 \times 2)$ simulation box sII hydrate with methane-ethane-propane guest molecules. Methane is shown in cyan; ethane in green; propane in yellow. The red lines are hydrate cavities. 


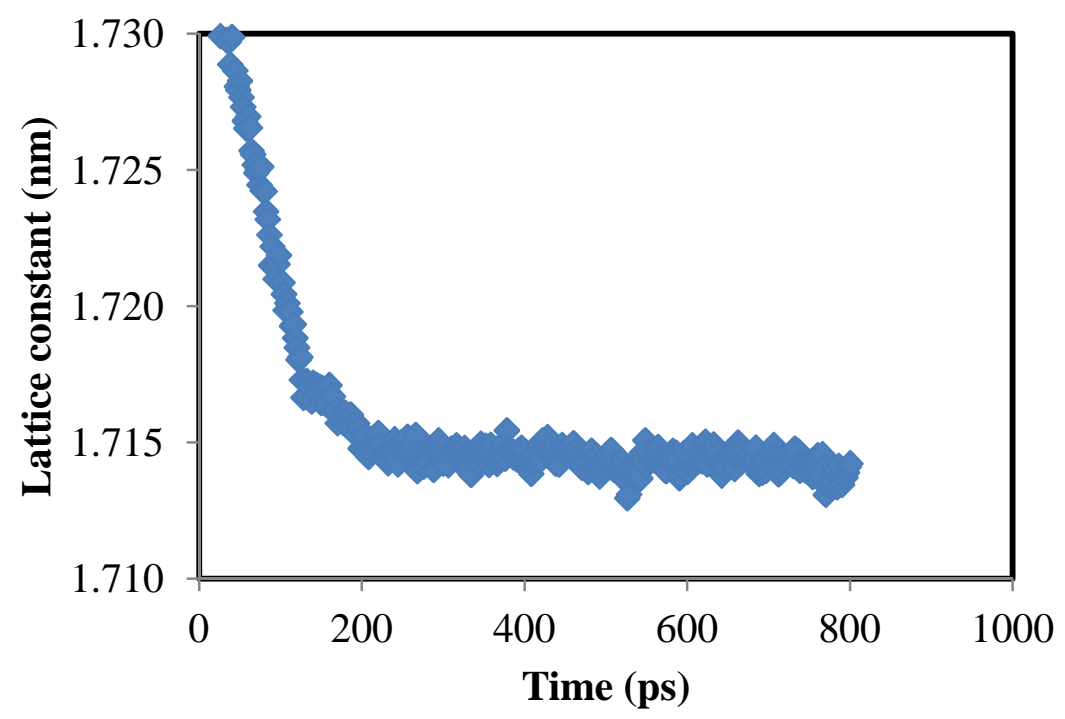

Figure 3.4 Equilibriation of lattice constant for $2 \times 2 \times 2$ sII hydrate unit cells at $260 \mathrm{~K}$

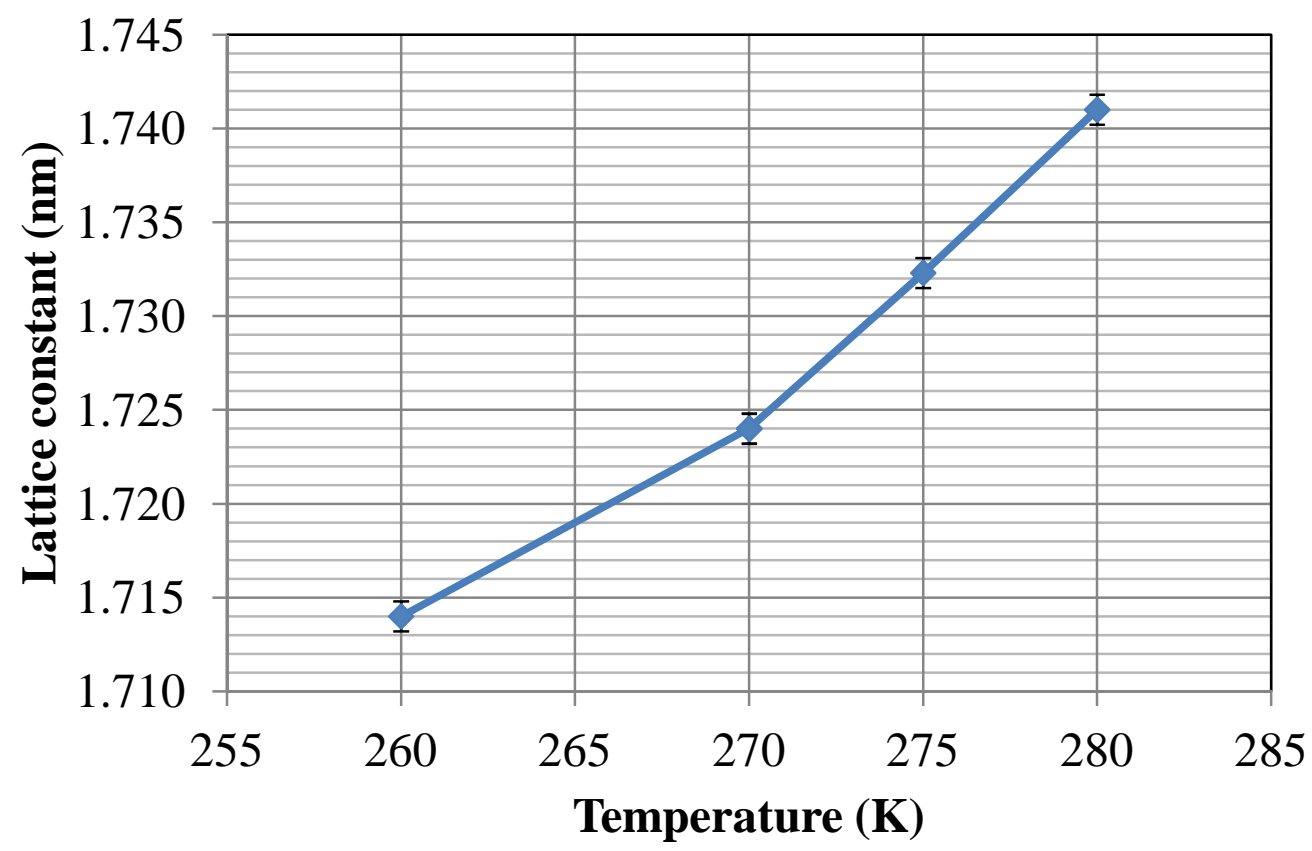

Figure 3.5 Effect of temperature on the lattice constant of sII hydrate. The experimental value at $275 \mathrm{~K}$ is $1.738 \mathrm{~nm}$ and the value obtained by simulation is $1.732 \mathrm{~nm}$. 


\subsection{Simulation configuration for dissolution}

To study the dissolution of sII mixed hydrate, a simulation box containing $4 \times 4 \times 3$ unit cell of sII hydrate was constructed based on the neutron diffraction studies for the oxygen of water positions and by the Bernal-Fowler rules for the orientation of hydrogen atoms. The lattice constant for sII hydrate is $17.32 \AA$. The dimensions of the hydrate simulation box are $6.92 \times 6.92 \times 5.196 \mathrm{~nm}^{3}$. The critical cluster size for the nucleation predicted by the classical nucleation theory is $32 \AA^{127}$ and by Radhakrishna and Trout ${ }^{54}$ based on the Landau-Ginzburg free energy calculations for $\mathrm{CO}_{2}$ clathrate hydrate is in between 9.6 and $14.5 \AA$. The above critical size of nucleation given is for sI hydrate. The sI hydrate has a lattice constant of $12.03 \AA$, so a 32 unit cell $(4 \times 4 \times 2)$ can be subcritical in one of the direction of the simulation cell. This means that for running the dissolution or dissociation studies, the size of the simulation box should be more than the critical size of nucleation. A 48 unit $(4 \times 4 \times 3)$ cell or a 64 unit cell $(4 \times 4 \times 4)$ will be above the critical size of nucleation. Applying similarly to the sII hydrates, we assume that a 48 unit cell will be above the critical size of nucleation. We used a 48 unit cell $(4 \times 4 \times 3)$ simulation box in this work for all the dissolution simulations.

The initial configuration of the hydrate box for the dissolution simulations was obtained by appending a cell of 10946 water molecule to the 48 unit cell $(4 \times 4 \times 3)$ in the $z$-direction such that the hydrate-water interface is along $x-y$ plane. The final simulation box obtained after adding the water cell is $6.92 \times 6.92 \times 12.95 \mathrm{~nm}^{3}$. A snapshot of the initial configuration simulation box is shown in Figure 3.6. The configuration of liquid water layer was obtained from an energy minimized liquid water layer simulation box followed by equilibration run for $1 \mathrm{~ns}$ at a temperature of $275 \mathrm{~K}$ and 1 bar pressure before combining to the hydrate structure. After the water cell is appended to the 
hydrate phase, the simulation box is energy minimized using the Steepest-Decent algorithm in Gromacs ${ }^{128}$. This is done to reduce the thermal noise in the structure and potential energies.

Hydrocarbon guest molecules were added to the center of the cages. In a sII unit cell, there are 16 small cages and 8 large cages. For a fully occupied hydrate cages in a unit cell, all 16 small cages were occupied with methane molecules and 8 in large cages, 2 cages were occupied by methane, 4 cages were occupied by ethane and 2 cages were occupied by propane molecules. So in a 48 unit cell for fully occupied hydrate there are 768 methane molecules in small cages, 96 methane molecules in large cages, 192 ethane molecules in large cages and 96 propane molecules in large cages. The concentration of the guest gases in the hydrate phase were changed by varying the cage occupancy of the gas molecules. Models were constructed by varying the cage occupancy of ethane and propane in the large cage, methane cage occupancy both in the small and large cages. The effect of small cage occupancy was studied by constructing the models for methane occupancy of $100 \%$ and $81.25 \%$ in small cages. The naturally occurring hydrates have small cage occupancy around $90 \%$ and we want to have lower than the $90 \%$ occupancy. The empty cages were distributed all along the hydrate by removing gas molecules approximately from each unit cell. The details of all the different models constructed are tabulated in a Table 3.6. 


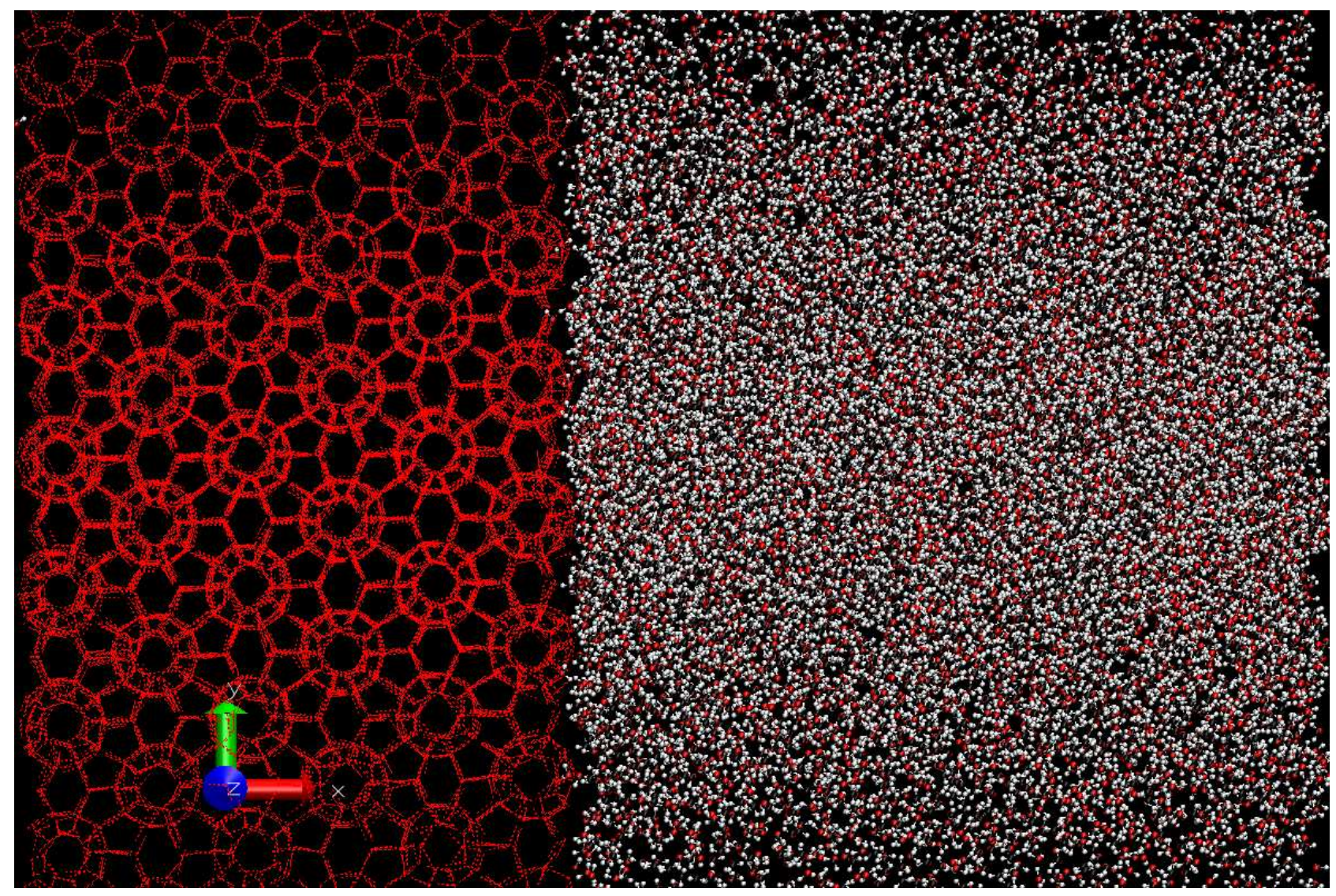

Figure 3.6 Snap shot of the initial simulation box showing the hydrate-water interface. Hydrate is shown in red color lines (hydrogen bonded lines). Liquid water layer: water of oxygen is in red color and hydrogen in white

All simulations were performed in a NPT ensemble in which the temperature and pressures were kept constant. Simulations were performed at $275 \mathrm{~K}$ and 50 bar. At this temperature and pressures the sII mixed hydrate with methane-ethane-propane mixture is in the hydrate stability zone. After the simulation box was energy minimized the simulations were run in NVT ensemble for $15 \mathrm{ps}$ to equilibrate the temperature along the simulation box. In the NVT short run simulations the temperature was controlled by berendsen with a relaxation time of $0.1 \mathrm{ps}$. This is followed by the production run with NPT simulations for $30 \mathrm{~ns}$. All NPT simulations were performed with a time step of 1.5 fs and temperature and pressure were controlled by Nòse-Hoover ${ }^{129}$ thermostat $^{2}$ 
and Parrinello-Rahman ${ }^{130}$ barostat respectively. The Nòse-Hoover ${ }^{129}$ thermostat and Parrinello-Rahman ${ }^{130}$ barostat give better results but the system has to be close to the desired temperature. If the system is far away from equilibrium then there might be chances that the simulation box might blowup. To avoid these, the simulation box is equilibrated for temperature in NVT ensemble with berendsen thermostat. SHAKE ${ }^{131}$ constrain was used to maintain the geometry of the water molecules with a relative tolerance of $10^{-4} \mathrm{~nm}$. 
Table 3.6 Cage occupancies in the sII hydrate and number of guest gas molecules in the simulations

\begin{tabular}{|c|c|c|c|c|c|c|c|c|c|c|}
\hline & \multicolumn{2}{|c|}{ Small Cage } & \multicolumn{8}{|c|}{ Large Cage } \\
\hline & & & mol & $\begin{array}{l}\text { Imber } \\
\text { ules i } \\
\text { cage }\end{array}$ & arge & & & & & \\
\hline & $\mathrm{C}_{1}$ & $\mathrm{C}_{1} \%$ & $\mathrm{C}_{1}$ & $\mathrm{C}_{2}$ & $\mathrm{C}_{3}$ & $\begin{array}{l}\text { Total large } \\
\text { cage } \\
\text { molecules }\end{array}$ & $\mathrm{C}_{1} \%$ & $\mathrm{C}_{2} \%$ & $\mathrm{C}_{3} \%$ & $\begin{array}{l}\text { Total large } \\
\text { cage } \\
\text { occupancy }\end{array}$ \\
\hline Model1 & 768 & 100 & 96 & 192 & 96 & 384 & 100 & 100 & 100 & $100 \%$ \\
\hline Model2 & 768 & 100 & 96 & 192 & 48 & 336 & 100 & 100 & 50 & $88 \%$ \\
\hline Model3 & 768 & 100 & 96 & 192 & 24 & 312 & 100 & 100 & 25 & $81 \%$ \\
\hline Model4 & 768 & 100 & 96 & 96 & 48 & 240 & 100 & 50 & 50 & $63 \%$ \\
\hline Model5 & 768 & 100 & 96 & 48 & 48 & 192 & 100 & 25 & 50 & $50 \%$ \\
\hline Model6 & 624 & 81.25 & 96 & 192 & 96 & 384 & 100 & 100 & 100 & $100 \%$ \\
\hline Model7 & 624 & 81.25 & 48 & 192 & 96 & 336 & 50 & 100 & 100 & $88 \%$ \\
\hline Model8 & 624 & 81.25 & 48 & 96 & 48 & 192 & 50 & 50 & 50 & $50 \%$ \\
\hline Model 9 & 768 & 100 & 96 & 119 & 96 & 311 & 100 & 61.97 & 100 & $81 \%$ \\
\hline Model10 & 768 & 100 & 24 & 192 & 96 & 312 & 25 & 100 & 100 & $81 \%$ \\
\hline Model11 & 768 & 100 & 96 & 192 & 2 & 290 & 100 & 100 & 2.08 & $76 \%$ \\
\hline Model12 & 768 & 100 & 96 & 98 & 96 & 290 & 100 & 51.04 & 100 & $76 \%$ \\
\hline
\end{tabular}

$\mathrm{C}_{1}=$ methane, $\mathrm{C}_{2}=$ ethane, $\mathrm{C}_{3}=$ propane 


\subsection{Results}

Dissolution of sII hydrate in the presence of water layer was carried out for $30 \mathrm{~ns}$ simulation time. Figure 3.7 shows the number density plot of the hydrocarbon guest in the hydrate layer along the $\mathrm{z}$-direction of the simulation box before the start of simulation $(0 \mathrm{~ns})$ and after $30 \mathrm{~ns}$ simulation time. Figure 3.8 shows the snapshot of the simulation box at the end of the simulation. Figure 3.7 is for Model 1 in which all the cages are fully occupied. From the Figure 3.6, we can see that there is no much change in the hydrate phase after $30 \mathrm{~ns}$ simulations. There were no ethane or propane molecules found to be in the liquid phase, only a few methane molecules moved in to the liquid phase from the hydrate phase. 

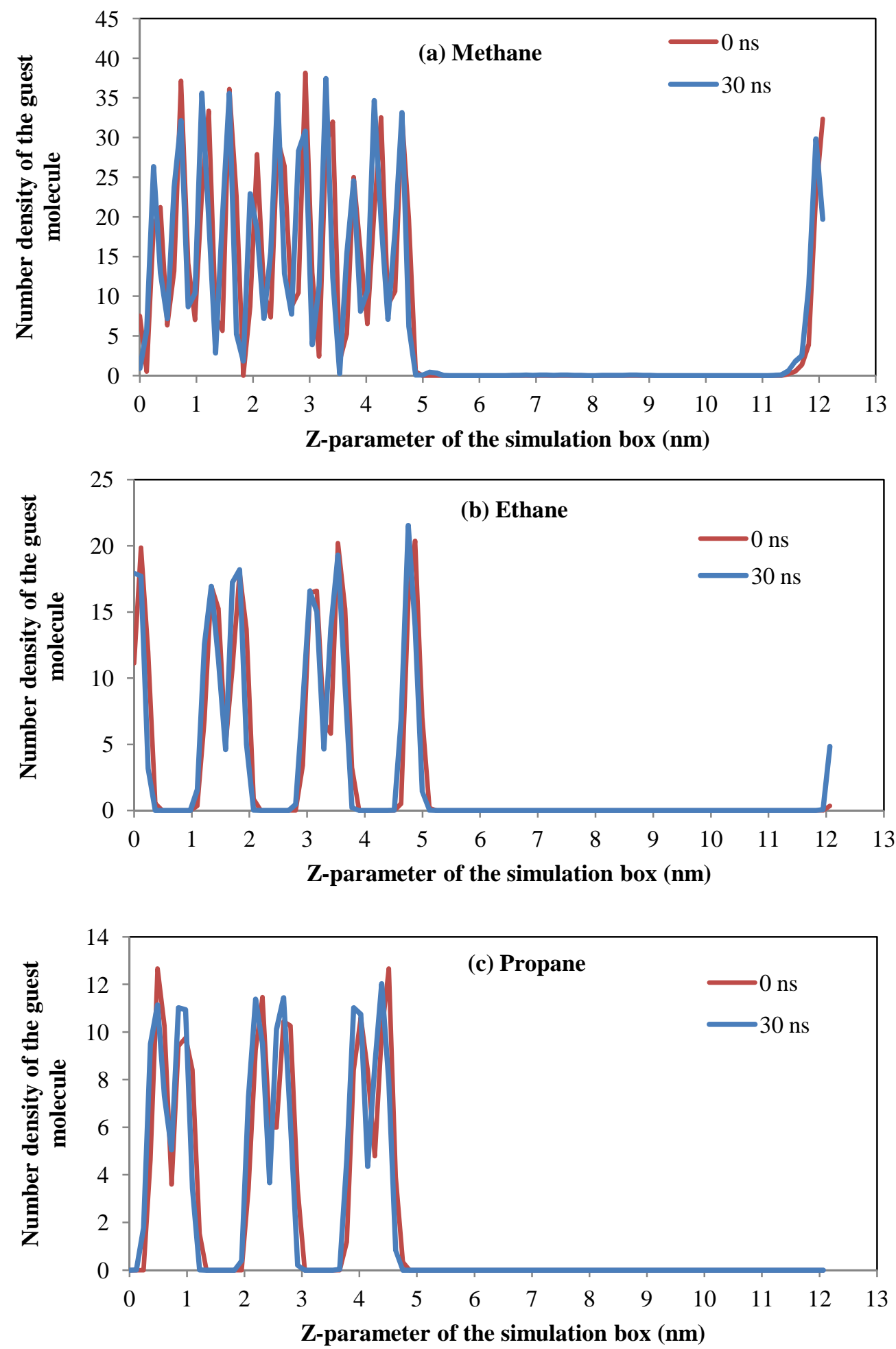

Figure 3.7 Density plot of guest gas molecules across the simulation box to view the change in number density of the gas molecules at $0 \mathrm{~ns}$ and at $30 \mathrm{~ns}$ simulations for (a) methane, (b) ethane (c) propane. 


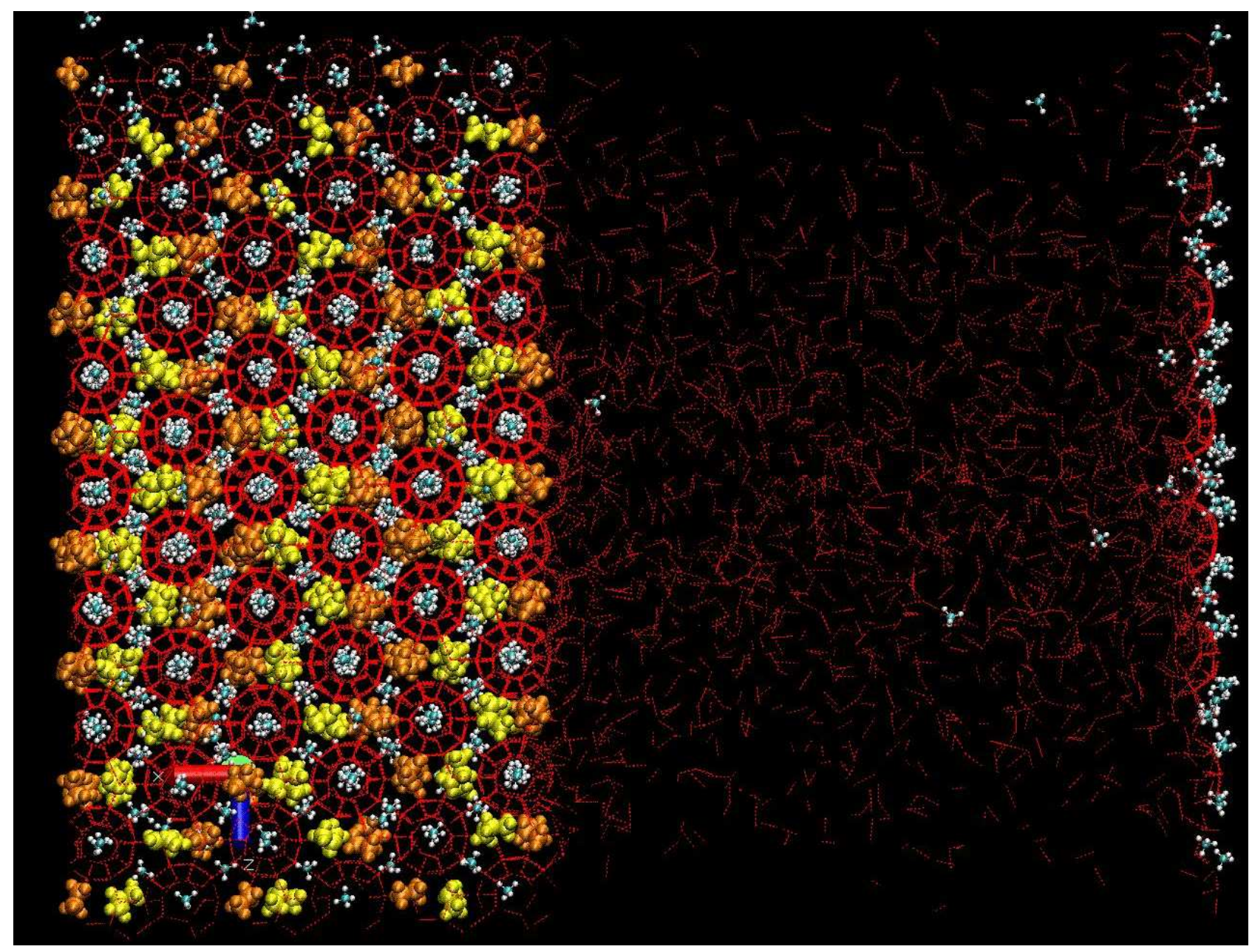

Figure 3.8 Snapshot of the sII mixed hydrate-water system simulation box containing methane, ethane and propane guest molecules for Model 1. Methane molecules are colored in cyan, ethane molecules are colored in orange, propane molecules are colored in yellow. Water molecules are shown in red lines as hydrogen bonds.

\subsubsection{Effect of Large cage occupancy}

Models 1 to Model 5 are for fully occupied small cages and with different large cage occupancy obtained by varying the ethane and propane guest gas molecules. Model 1 is for the cages which are $100 \%$ occupied. Model 1 considered here is as the base case and all other models are constructed in reference to the Model 1. Model 2 is $50 \%$ of propane obtaining $88 \%$ occupancy in large cage and Model 3 is with $25 \%$ propane which obtained $81 \%$ occupancy in large cage. In 
Model 4 and in Model 5, both ethane and propane occupancy is varied obtaining 63\% and 50\% occupancy in large cage. Model 4 is with 50\% ethane and 50\% propane and Model 5 is obtained with $25 \%$ ethane and $50 \%$ propane.

The amount of methane gas dissolved in the liquid water from hydrate phase is found to increase with the decrease in the cage occupancy of large cage for models 1 to 5 . Figure 3.9 shows the number of methane molecules dissolved in the liquid water from hydrate phase with the change in cage occupancy in large cages. The amount of dissolution of methane is lower at $100 \%$ cage occupancy i.e. hydrate is more stable when all the cages are fully occupied than with the presence of empty cavities. The increase in the dissolution of methane with decrease in cage occupancy might be because of the presence empty cages in the hydrate crystal which causes the destabilization of the hydrate lattice. Similar effect was found by Mysakhin et al. ${ }^{56}$ for sI methane hydrate dissociation study where the rate of dissociation of methane was higher with the decrease in the cage occupancy.

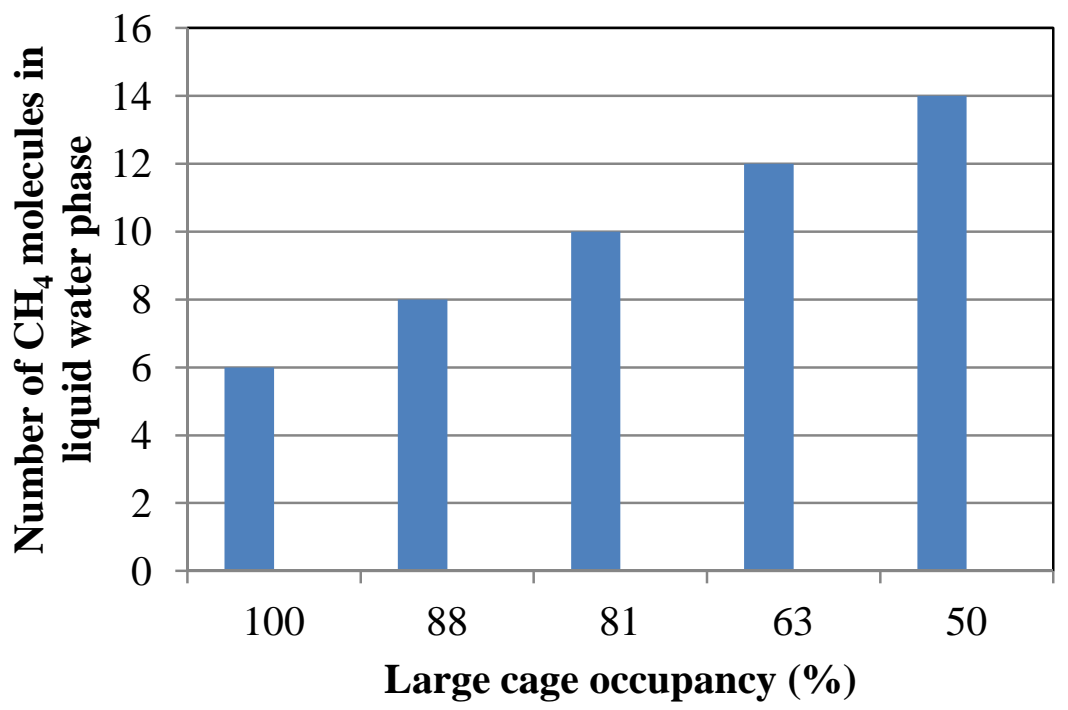

Figure 3.9 The amount of methane molecules moved in to the water phase from sII hydrate phase at different large cage occupancies (Model 1 to Model 5). 
More dissolution of methane was found in the case of 50\% occupancy in large cage (Model 5). Figure 3.10 is the snapshot of the simulation at the end of $30 \mathrm{~ns}$. Figure 3.10 shows the number density plot of methane, ethane and propane in the hydrate phase at $0 \mathrm{~ns}$ and $30 \mathrm{~ns}$. From the number density plot of methane (Figure 3.11a), it can be observed that there is variation of the peaks on either side of the hydrate-water interface. A decrease in the height of the peak at the hydrate-water interface on either sides of the hydrate phase is an indication of hydrate dissolution process which causes the interface to move in to the hydrate phase. 


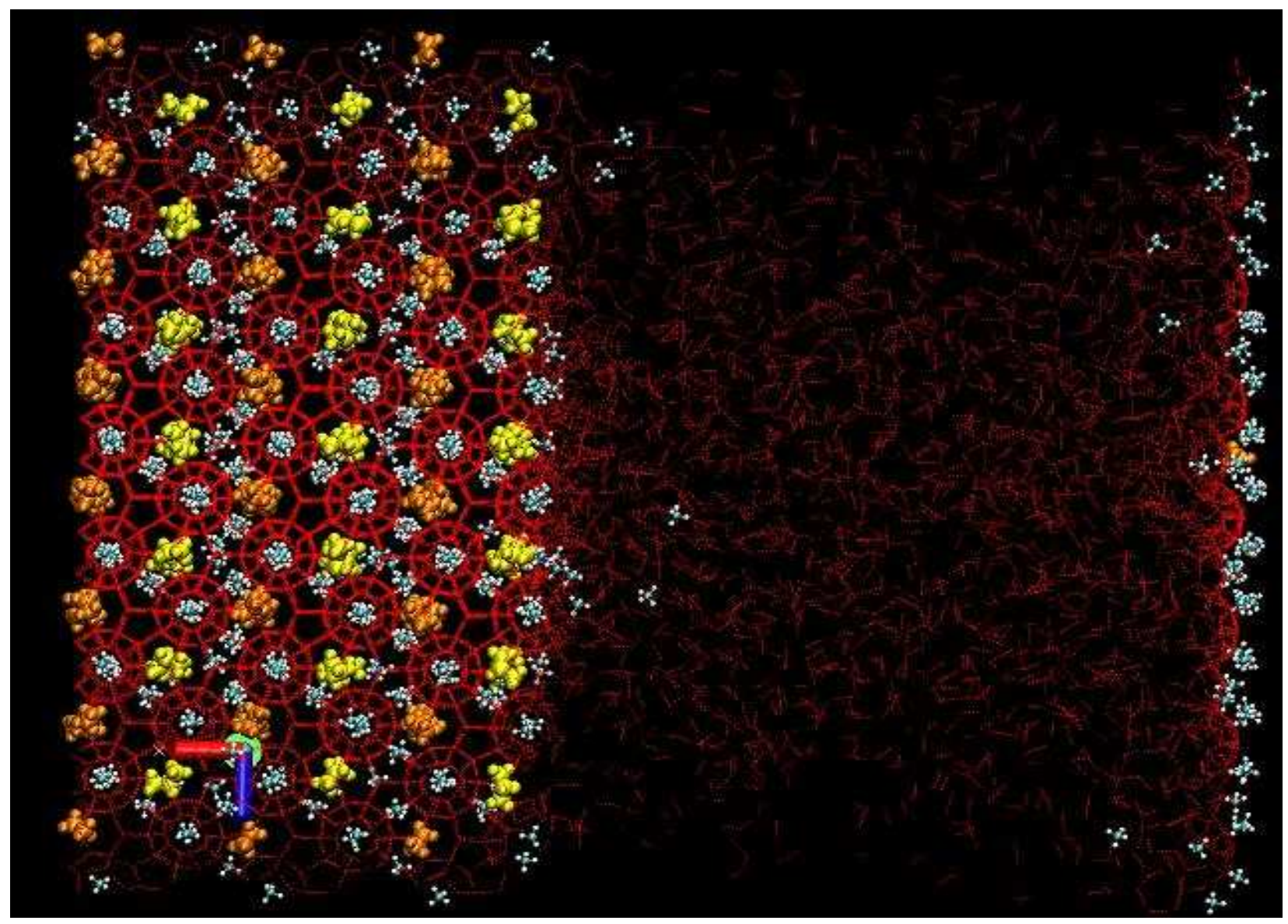

Figure 3.10 Snapshot of the simulation box for Model 5 at the end of simulation. Methane molecules are colored in cyan, ethane molecules are colored in orange, propane molecules are colored in yellow. Water molecules are shown in red lines as hydrogen bonds. 

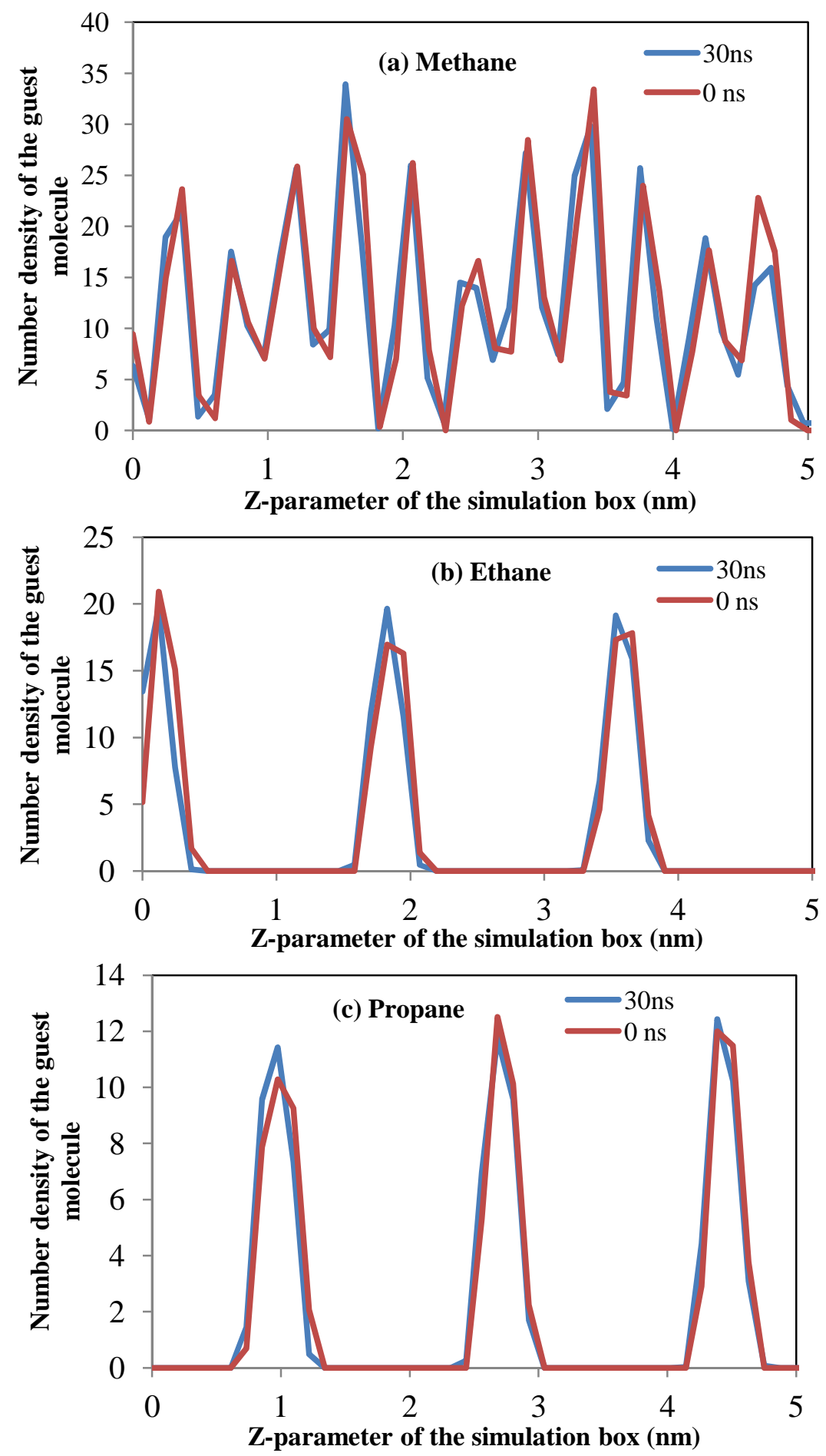

Figure 3.11 Number density plot of the guest molecules in the hydrate phase along the z-direction of the box. (a) methane, (b) ethane (c) propane 
The rate of methane released in to the bulk liquid water was also calculated based on the $F$ value in which water molecules were classified as liquid or solid-like. Figure 3.12 shows the number of methane molecules that dissolve in to the liquid water from hydrate phase for different large cage occupancy (Model 1 to Model 5). It can be seen from Figure 3.12 that the rate of dissolution is not a steady change, the methane population shows oscillatory behavior throughout the simulation. This oscillatory behavior is a consequence of the hydrate breakage and hydrate reformation. Visual inspection of trajectories reveals that the methane molecules oscillate at the hydrate-liquid water interface moving in and out of the partial cages.

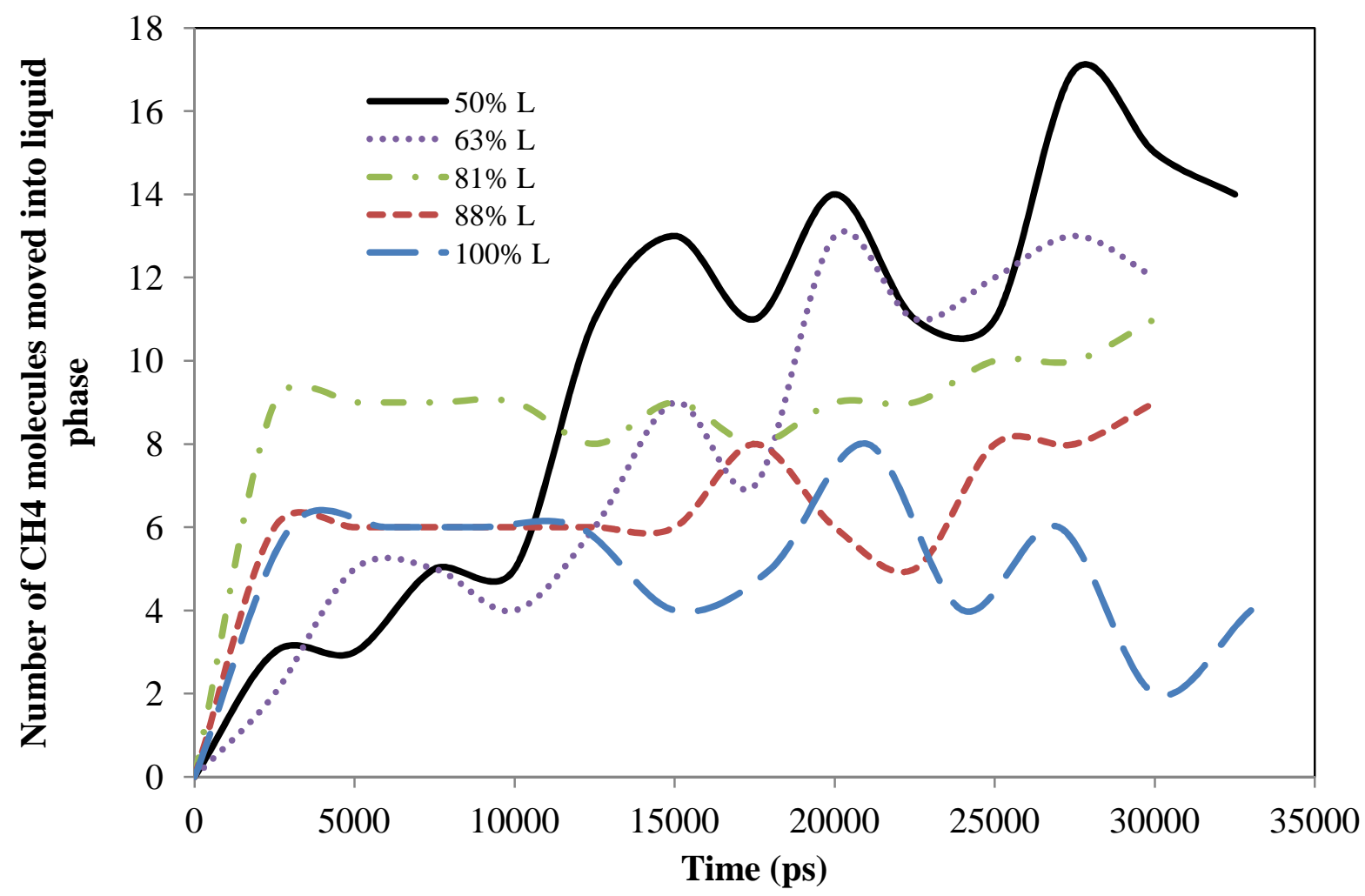

Figure 3.12 Number of $\mathrm{CH}_{4}$ molecules moved in to the bulk liquid water from hydrate phase during the course of the simulation at different large cage occupancy (L). The small cage is $100 \%$ occupied. Model 1-100\%, Model 2-88\%, Model 3-81\%, Model 4-63\%, Model 5-50\% large cage occupancy 


\subsubsection{Effect of small cage occupancy}

The effect of small cage occupancy on the stability of sII hydrate was also studied. Model 6, Model 7 and Model 8 are with $81.25 \%$ small cage occupancy and with 100\%, 88\% and 50\% occupancy in large cages respectively. The large cage occupancy obtained in Model 7 is by emptying methane in large cage (25\% methane). The Model 8 large cage occupancy is obtained by $50 \%$ methane, $50 \%$ ethane and 50\% propane. Figure 3.14 shows the dissolution of methane from hydrate phase to liquid water phase at $81.25 \%$ small cage occupancy and different large cage occupancy. The dissolution of methane molecules displays oscillatory behavior, similar to what was observed in the Models 1 to 5 . Figure 3.13 shows the number of methane molecules moved in to the liquid phase for $100 \%$ and $81.25 \%$ small cage occupancy. Though the percentage of large cage occupancy is same for the two cases, $100 \%$ and $81.25 \%$ small cage occupancy, considered in Figure 3.13, the composition of methane, ethane and propane are different in large cages (see Table 3.6).

The number of methane molecules released in to the water is almost same in the case of $100 \%$ and $81.25 \%$ small occupancy. The small difference in the two cases suggests that the dissolution of methane gas in to the liquid water does not change significantly when the small occupancy of sII hydrate is reduced from $100 \%$ to $81.25 \%$. 


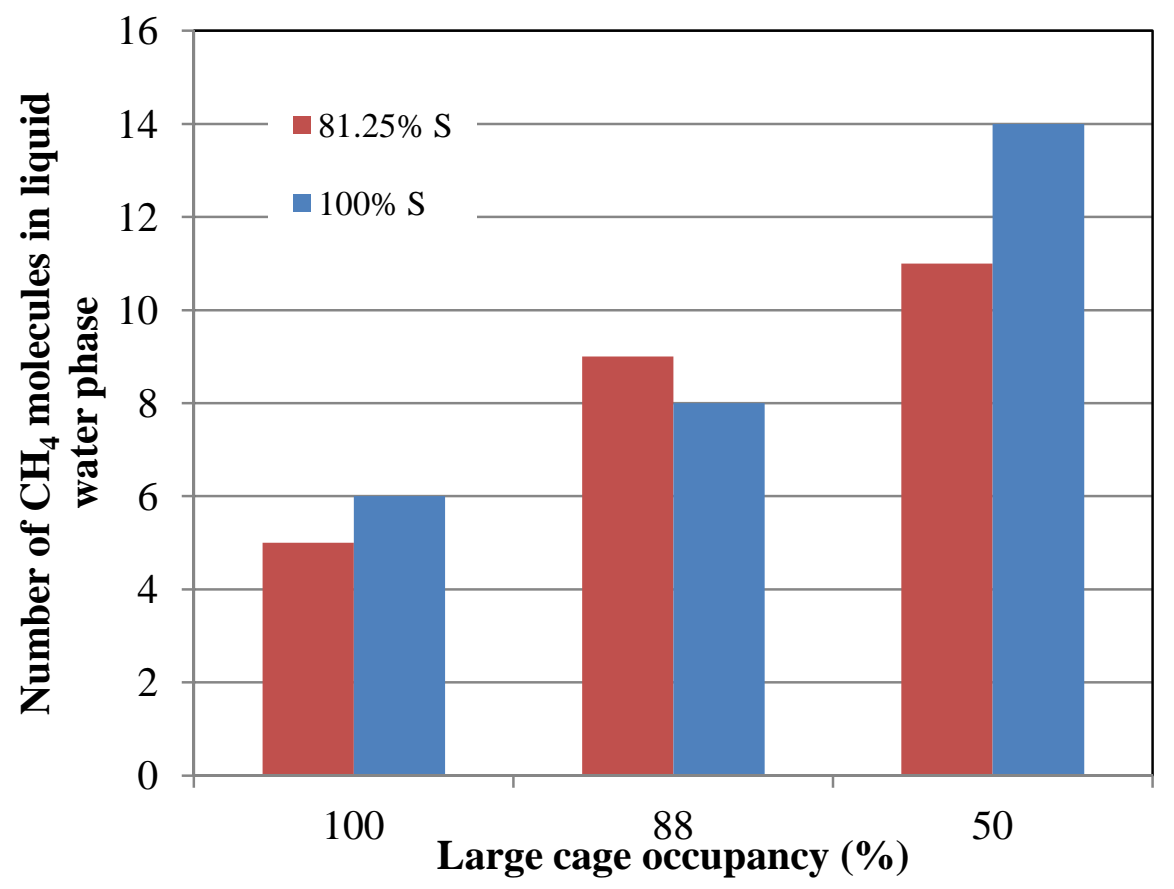

Figure 3.13 Effect of small cage occupancy (S) on the dissolution of methane molecules to the liquid water phase from the hydrate phase for 100\% small cage occupancy (Model 1, Model 2 and Model 5) and 81.25\% small cage occupancy (Model 6, Model 7 and Model 8).

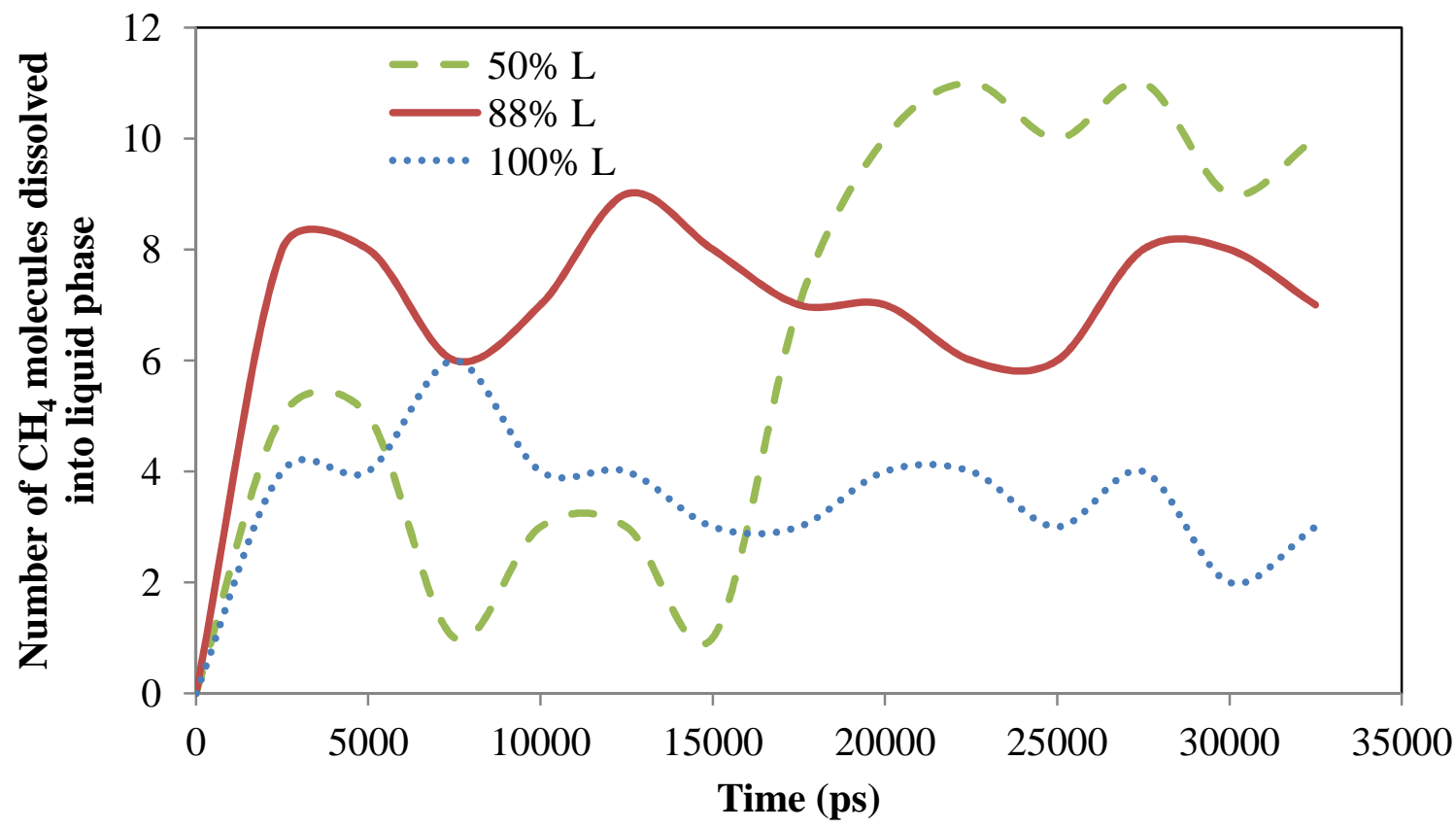

Figure 3.14 Number of $\mathrm{CH}_{4}$ molecules that moved in to the bulk liquid water from hydrate phase during the course of the simulation at $81.25 \%$ small cage occupancy and at different large cage occupancy (L) 


\subsubsection{Effect of ethane and propane}

MD simulations were also performed by varying the methane, ethane, propane gas composition in the large cage by keeping the overall cage occupancy same. Model 3, Model 9 and Model 10 are for $81 \%$ occupancy in large cage. The $81 \%$ large cage occupancy obtained by Model 3 is with 25\% propane, Model 9 with $62.5 \%$ ethane and Model 10 with 25\% methane in large cage. Model 11, Model 12 and Model 13 are for 76\% occupancy in large cage. Model 11 is obtained by $2 \%$ propane and Model 13 by $51 \%$ ethane. We did not observe any clear trend in the dissolution phenomena by varying the guest specific composition for $81 \%$ occupancy case and $76 \%$ occupancy case. Figure 3.15 and 3.16 shows the dissolution of methane molecules in liquid water for $81 \%$ occupancy and $76 \%$ occupancy. If the amount of gas dissolved is dependent only on the vacant cavities then the amount of methane dissolved should be same but the dissolution rates are different for different guest composition. Thus, the presence of higher hydrocarbons has some effect on the stability of hydrate. From Figure 3.15 we can observe that more number of methane dissolved in to the liquid water phase is in the case of Model 3 (propane changed) followed by Model 10 (Ethane changed) and by Model 9 (methane changed in large cage). Figure 3.15 shows the dissolution of methane during the course of the simulation for $76 \%$ occupancy in large cage. 


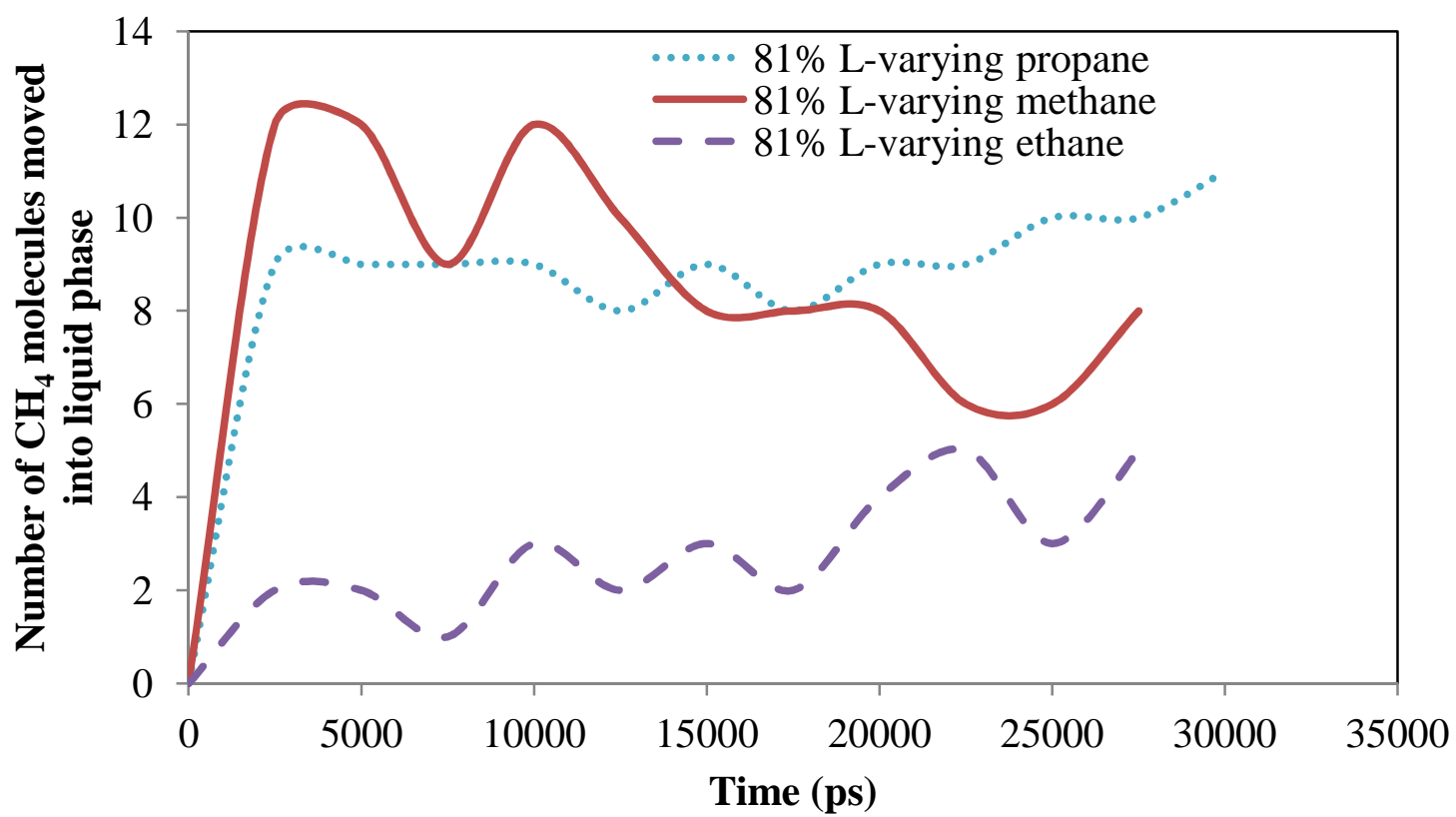

Figure 3.15 Number of $\mathrm{CH}_{4}$ molecules that moved in to the bulk liquid water from hydrate phase during the course of the simulation at $81 \%$ large cage occupancy. L-denotes for large cage occupancy.

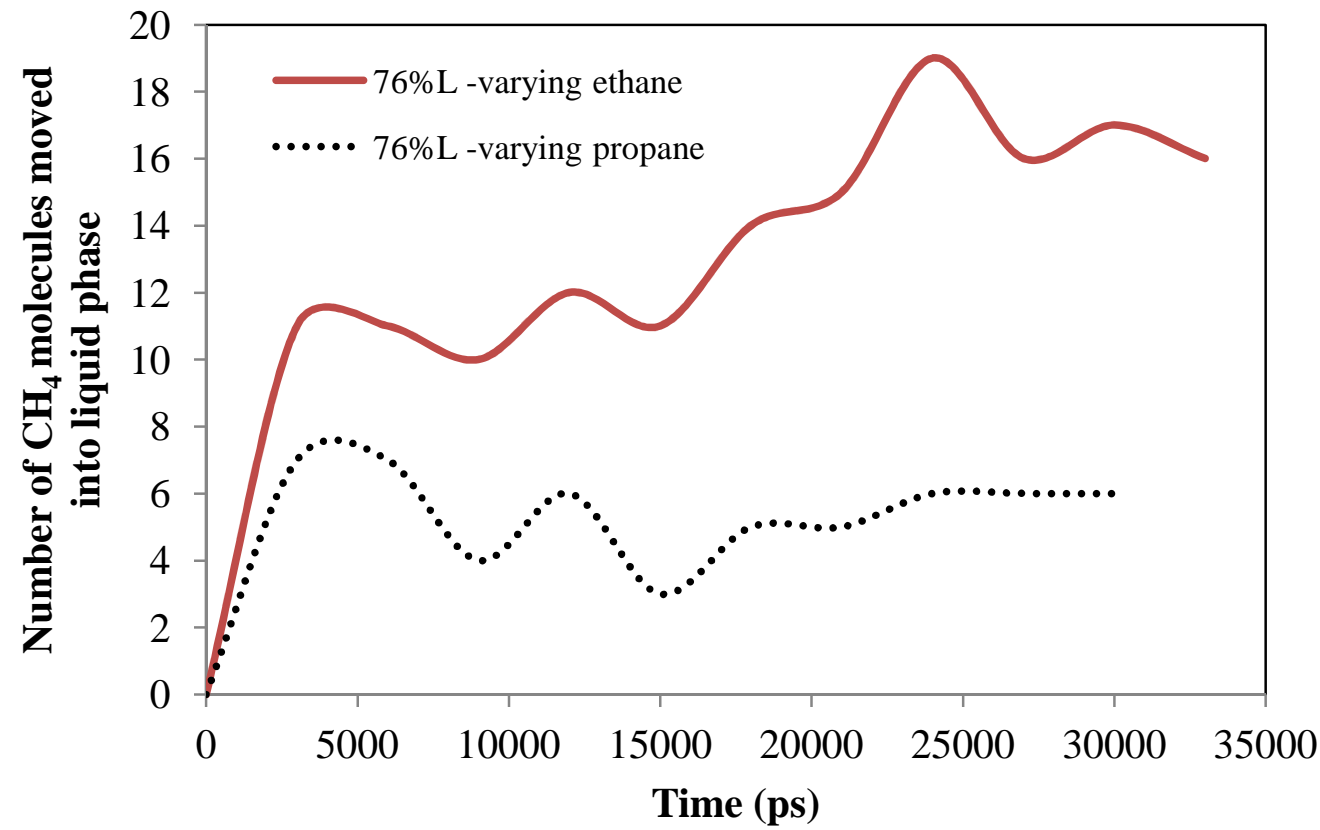

Figure 3.16 Number of $\mathrm{CH}_{4}$ molecules that moved in to the bulk liquid water from hydrate phase during the course of the simulation at $76 \%$ large cage occupancy. L-denotes for large cage occupancy 
The dissolution results for the models considered are summarized in Table 3.7. In all the simulations, we observed that none of the ethane or propane molecules moved in to the bulk of liquid water. There are only methane molecules which moved in to the liquid water phase from the hydrate phase. The number of molecules that moved in to liquid phase was calculated from the average of last 4 ns simulation.

Table 3.7 Effect of cage occupancy on the dissolution of sII hydrate

\begin{tabular}{|c|c|c|c|c|c|c|c|}
\hline & Small cage & & arge cag & & & & \\
\hline & $\begin{array}{c}\text { Methane } \\
\%\end{array}$ & $\begin{array}{c}\text { Methane } \\
\%\end{array}$ & $\begin{array}{c}\text { Ethane } \\
\%\end{array}$ & $\begin{array}{c}\text { Propane } \\
\%\end{array}$ & $\begin{array}{c}\text { Large cage } \\
\text { occupancy } \\
(\%)\end{array}$ & $\begin{array}{c}\text { Total cage } \\
\text { occupancy } \\
\%\end{array}$ & $\begin{array}{l}\text { Number of } \\
\text { methane } \\
\text { molecules } \\
\text { in water } \\
\text { phase }\end{array}$ \\
\hline Model 1 & 100 & 25 & 50 & 25 & 100 & 100 & 6 \\
\hline Model 2 & 100 & 25 & 50 & 12.5 & 88 & 95.8 & 8 \\
\hline Model 3 & 100 & 25 & 50 & 6.25 & 81 & 93.7 & 10 \\
\hline Model 4 & 100 & 25 & 25 & 12.5 & 63 & 87.5 & 12 \\
\hline Model 5 & 100 & 25 & 12.5 & 12.5 & 50 & 83.3 & 15 \\
\hline Model 6 & 81.25 & 25 & 50 & 25 & 100 & 87.5 & 5 \\
\hline Model 7 & 81.25 & 12.5 & 50 & 25 & 88 & 83.3 & 8 \\
\hline Model 8 & 81.25 & 12.5 & 25 & 12.5 & 50 & 70.8 & 11 \\
\hline Model 9 & 100 & 25 & 31 & 25 & 81 & 93.7 & 5 \\
\hline Model 10 & 100 & 6.25 & 50 & 25 & 81 & 93.7 & 12 \\
\hline Model 11 & 100 & 25 & 50 & 1.08 & 76 & 91.8 & 8 \\
\hline Model 12 & 100 & 25 & 26 & 25 & 76 & 91.8 & 16 \\
\hline
\end{tabular}




\subsubsection{Structure I methane hydrate}

MD simulations were performed on sI methane hydrate by Vedam ${ }^{132-133}$ to study the dissolution of methane as a function of percentage of small cage occupancy. The sI hydrate has 6 small cages and 2 large cages in a unit cell. These simulations were performed on a 64 unit cell for $35 \mathrm{~ns}$. The liquid layer next to the hydrate phase has 6917 water molecules. The number of methane molecules dissolved in to the liquid water layer is reported in Figure 3.17 for different small cage occupancies. The dissolution was found to increase as the cage occupancy is decreased from $100 \%$ occupancy to $25 \%$ small cage occupancy. A lower dissolution rate was found in the case of $0 \%$ occupancy. Figure 3.18 shows the number of methane molecules dissolved as a function of time for different small cage occupancy. The higher stability at $100 \%$ and $0 \%$ cage occupancy can be explained by their lack of crystal defects in the periodic crystal defects, while the other cage occupancies introduces crystal defects making them unstable. The methane population displays oscillatory behavior similar to what we have observed in our simulations.

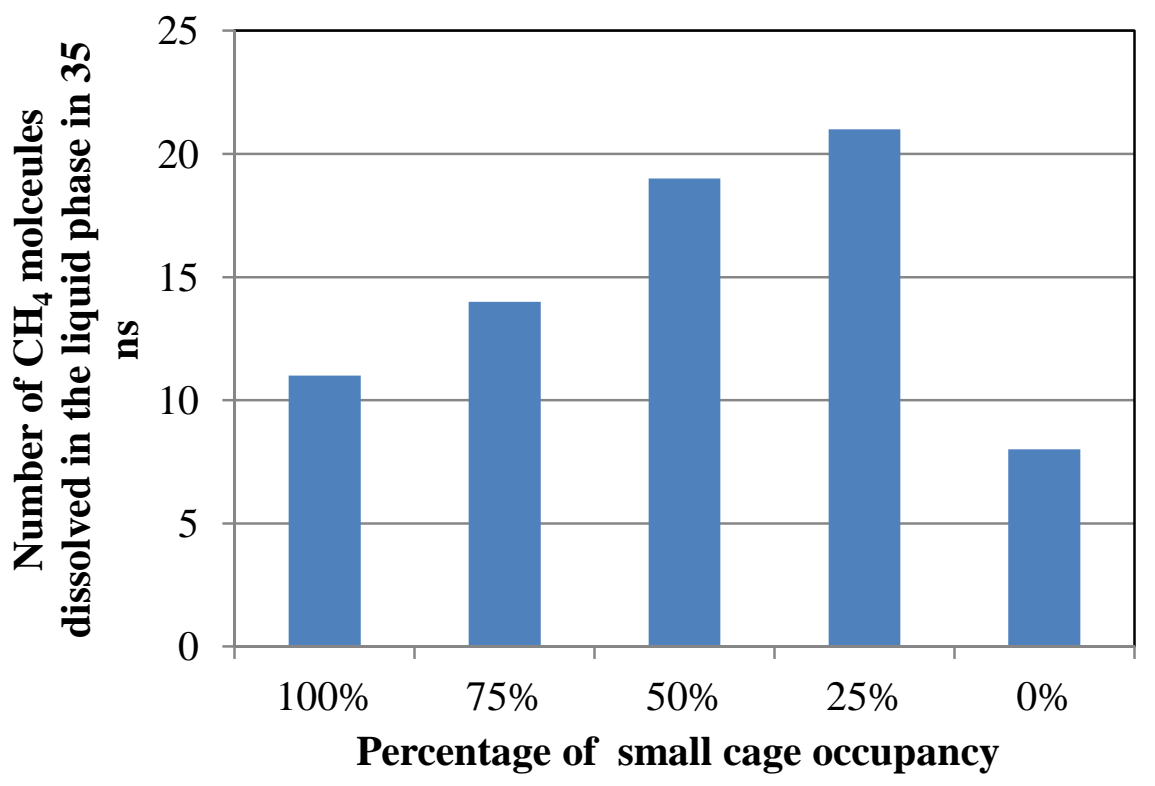

Figure 3.17 Number of methane molecules dissolved in to the liquid water for sI methane hydrate. 


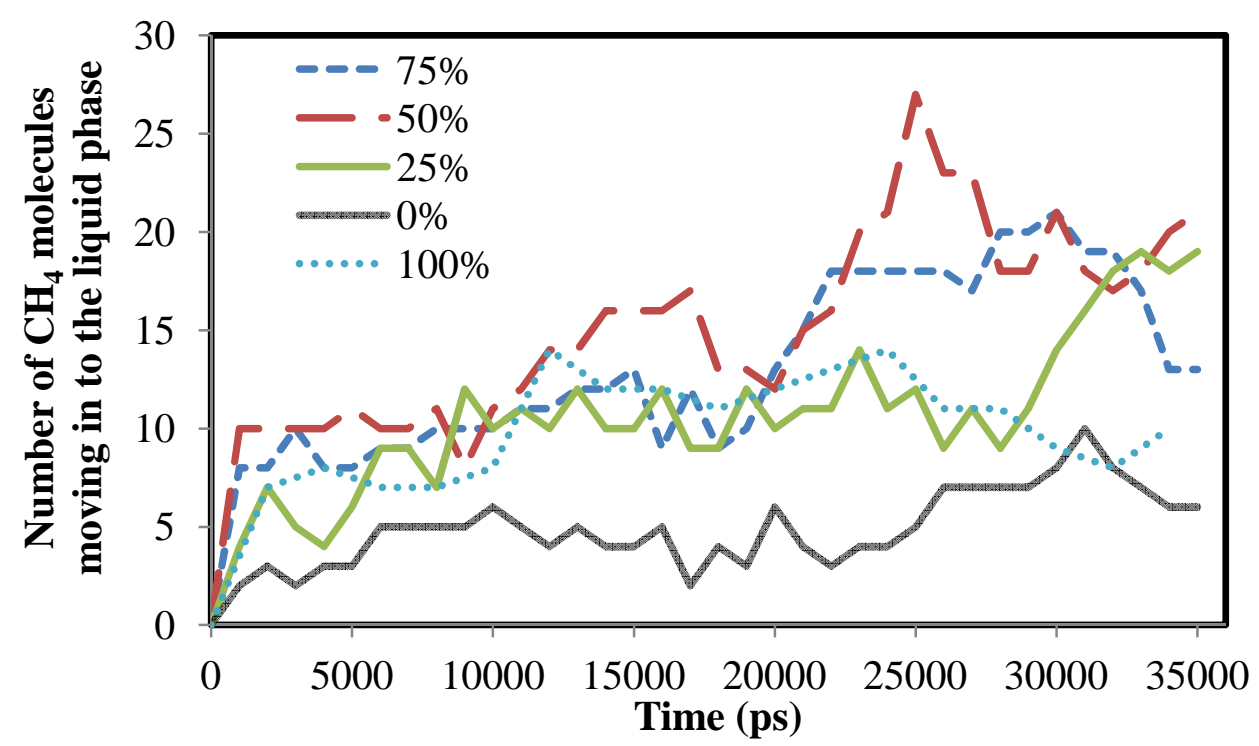

Figure 3.18 Number of methane molecules moved in to the liquid water during the course of the simulation at different percentage of cage occupancies for sI methane hydrate.

The amount of dissolution in the case of sI methane hydrate is higher than that of sII mixed hydrate (see Figures 3.9 and 3.17). In sI hydrate, a unit cell has 2 small cages and 6 large cages, where as in sII hydrate there are 16 small cages and 8 large cages in a unit cell. The small cages are more stable than the large cages. Most of the methane molecules that moved in to the liquid water are from the large cages. sI hydrate has more number of large cages and are occupied by methane, where as in sII mixed hydrate (methane ethane and propane mixture) there are lesser number of large cages in comparison to sI and are occupied by methane ethane and propane. These less occupancy of methane in large cage might be the reason for the lower dissolution of gas in to the liquid phase. 


\subsection{Discussion}

The hydrate dissolves because the surrounding water layer is under-saturated and it should continue till the saturation is achieved. There is a chemical potential driving force between the hydrate and the liquid water which makes the hydrate to dissolve. The saturation values are nothing but the solubility of gas in liquid. Here, there is a two phase hydrate $(\mathrm{H})$-liquid water $\left(\mathrm{L}_{\mathrm{w}}\right)$ region exits and there is no vapor phase. So the saturation values should be at $\mathrm{H}-\mathrm{L}_{\mathrm{w}}$ two phase region but not at the vapor-liquid water region. The calculation of the solubility of hydrate forming gas in water at two phase $\mathrm{H}-\mathrm{L}_{\mathrm{w}}$ equilibrium is described in the Chapter 4. At $275 \mathrm{~K}$ and 50 bar, the aqueous phase solubilities obtained using our model described in Chapter 4 in terms of molefraction for methane, ethane at $\mathrm{H}-\mathrm{L}_{\mathrm{w}}$ two phase region are 0.001275 and 0.0003179 . These solubility values are for pure methane and pure ethane hydrate. Pure propane do not form two phase hydrate at $275 \mathrm{~K}$ and 50 bar, but it forms mixed hydrate at $275 \mathrm{~K}$ and 50 bar conditions with ethane and methane. We have used the saturation value at $\mathrm{V}-\mathrm{L}_{\mathrm{w}}$ and the value is 0.000139 as there will not be much change in the molefraction value. The liquid water layer next to the hydrate layer has 10697 water molecules, so the corresponding number of molecules of methane, ethane and propane at saturation are 14, 3 and less than 2 respectively.

The results reported in Table 3.7 reveals that the dissolution of hydrate is sensitive to the cage occupancy. In all the simulations, we did not observe any of ethane or propane molecules dissolved in to the liquid water from the hydrate phase. There are only methane molecules which are dissolved in to the liquid water from the hydrate phase. The number of methane molecules dissolved has been increased with the decrease in the large cage occupancy from 100\% to 50\% (see Figure 3.8). The introduction of empty cages in the large cage of the sII crystal structure induces crystal defects in to the lattice destabilizing the structure, which promotes the methane molecules to 
move into the liquid water layer. Similar behavior was observed by earlier researchers ${ }^{60,113-114}$ for the dissociation of sI hydrate studies, where the dissociation of sI hydrate is a function of hydrate cage occupancy. From Figure 3.8 we can see that there is a steady increase in the number of methane molecules dissolved in to the liquid water with the change in large cage occupancy from $100 \%$ to $50 \%$. These results confirm that the fully occupied hydrate is more stable than the presence of empty cavities.

The dissolution of sII hydrate was studied by changing the cage occupancy of the small cage from $100 \%$ to $81.25 \%$. Figure 3.14 shows the comparison the methane dissolution for the two cases considered. There is no change in the amount of methane dissolved for $100 \%$ and $81.25 \%$ small cage occupancy indicating that the empty small cages have no effect on the dissolution of sII hydrate. The total cage occupancy in the cases of $81.25 \%$ small cage occupancy is less than that of $100 \%$ small cage occupancy. This means that there are more empty cages, and they are from small cages. If hydrates becomes unstable due to the vacant cages $^{56}$ then higher dissolution has to be observed in the case of $81.25 \%$ occupancy, as it has more vacant cages than that of $100 \%$ cases. Contrary to this, it was observed that there is no change in the dissolution with the change in small cage occupancy. Even in the previous studies on dissociation of sI methane hydrate it was observed that the small cages persists longer lifetime than that of large cages ${ }^{56,60}$. The small cages are more stable and the decrease in small cage occupancy to $81.25 \%$ (empty small cages) is not influencing the other cages to destabilize it. Also from Figure 3.19 for the same total cage occupancy of $87.5 \%$, the one that obtained by emptying the large cages has higher dissolution rate than the one obtained by emptying the small cages. This indicates that the stability of sII hydrate is more dependent on the cage occupancy of large cage than on the small cage occupancy, and therefore, the stability of hydrate is more to the cage specific occupancy than to the overall cage occupancy ${ }^{60}$. 


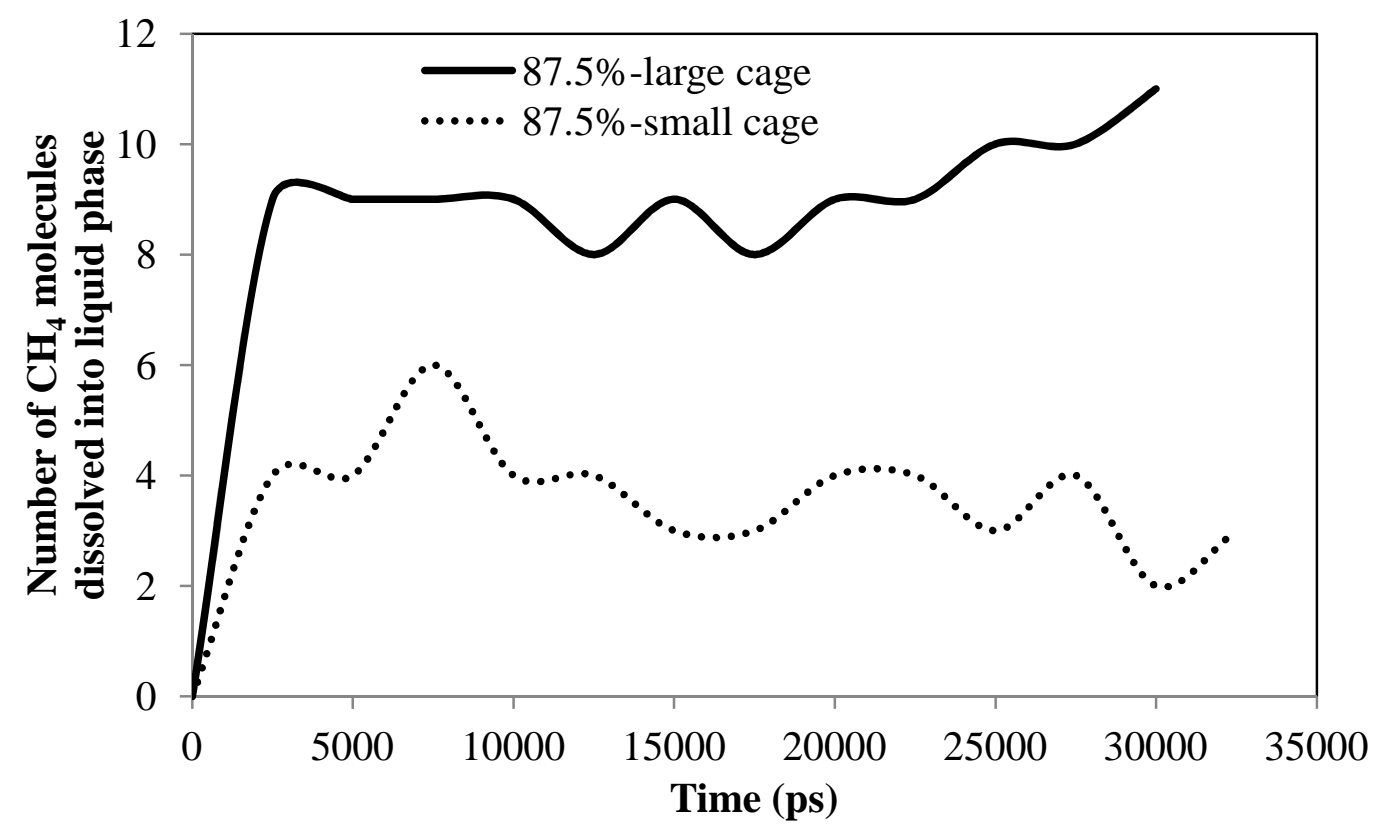

Figure 3.19 Dissolution of methane molecules from hydrate phase to the bulk liquid water during the course of the simulation. The lines are for total cage occupancy of $87.5 \%$ obtained by emptying small cage and by emptying large cage.

The difference in dissolution of sI and sII was explored to understand the role of crystal structure (sI vs sII) on the dissolution of the hydrate. Figure 3.17 shows the dissolution of methane from sI methane hydrate and Figure 3.10 for sII hydrate with varying cage occupancy. The amount of dissolution of methane in sI hydrate is more than the sII hydrate. Experimental studies ${ }^{30,43,45}$ on artificial methane hydrates and theoretical studies ${ }^{44}$ suggested that the dissolution of hydrate is diffusion-controlled phenomenon. If dissolution of the hydrate crystal is just the diffusioncontrolled and not influenced by difference in the hydrate crystal then the amount of methane dissolved in to the liquid phase has to be same. Here, higher rate of dissolution in sI compare to sII 
hydrate was observed suggesting that the crystal structure also have influence on the dissolution of hydrate.

The sII hydrate considered here is not a simple hydrate and is a mixed hydrate with ethane and propane occupied in the large cages along with methane. There was no ethane and propane molecules dissolved in to the liquid water phase was observed in all the simulations. The aqueous solubilities of ethane and propane are very less, and the numbers of ethane and propane molecules that can be dissolved are 3 and less than 2 respectively for the size of liquid water considered in the simulations. This lower solubility of ethane and propane can be one of the reasons that ethane and propane molecules are not dissolved in to the liquid water from hydrate phase. Similar to how methane molecules are more stable in the small cages, these propane and ethane molecules can also be stable in the large cages due to the size of the molecules. Further analysis is required to understand in detail the behavior of this extra stability of these gases.

To further understand the effect of ethane and propane on the dissolution of methane in to the liquid water, simulations were carried out by changing methane, ethane and propane occupancy in large cages keeping the overall occupancy same. Figure 3.15 and 3.16 shows the effect of ethane and propane cage occupancy on the dissolution of sII hydrate at $81 \%$ and $76 \%$ large cage occupancy. There was no clear trend in the dissolution process when varying the guest specific occupancy. Figure 3.20a shows the number of methane molecules dissolved as the propane occupancy is varied and Figure 3.20b is when ethane occupancy is varied. All other occupancies were kept same when varying propane or ethane occupancy. The dissolution of methane in to the liquid water was found to increase with the decrease in the propane occupancy upto $25 \%$ propane occupancy and then decrease for the $2 \%$ propane occupancy. In the case of ethane, there is no 
change in the dissolution of methane when ethane occupancy changed from $100 \%$ to $62.5 \%$ and then there is an increase when ethane occupancy is reduced to $51 \%$.
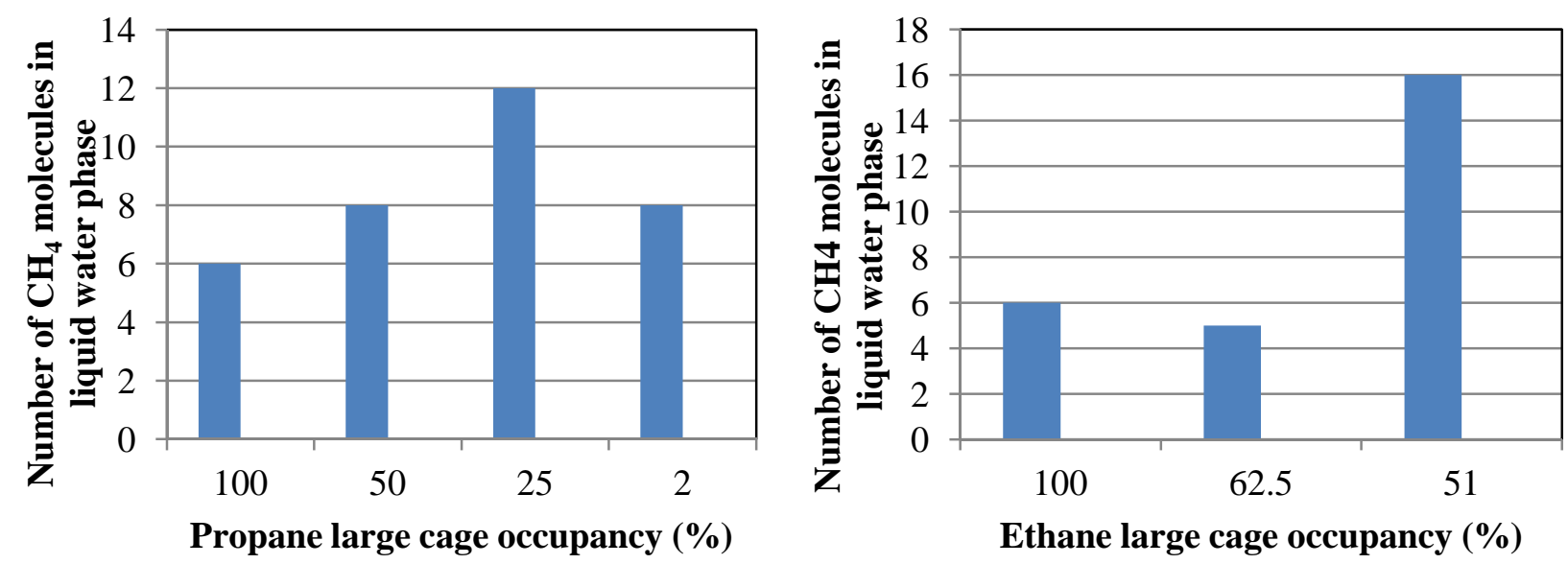

Ethane large cage occupancy $(\%)$

Figure 3.20 Dissolution of methane molecules into the liquid water as a function of (a) Propane large cage occupancy (b) Ethane large cage occupancy.

In all the simulations, the dissolution of methane in to the liquid water reveals an unusual behavior (Figure 3.12, 3.14, 3.15 and 3.16) at simulation times. There is a linear increase in the dissolution of methane in the first few nanoseconds of the simulation and then displays an oscillatory behavior. Visual inspection of the simulations using VMD, it was observed that the methane molecules are moving in and out of the hydrate crystals at the interface. We have also observed the increase in the hydrate like water molecules in the hydrate phase using the $F$ value (phase recognition) criteria. The methane molecules fluctuate at the interface moving in and out of the open water cages which are connected to the rest hydrate core. During the simulations these open cages grow increasing the hydrate-like water molecules at the interface. Figure 3.21 shows the number of water molecules in the hydrate phase during the course of the simulations. The increase in water molecules in the hydrate phase is more at higher cage occupancy. The hydrate is more 
stable at higher cage occupancy and the methane molecules at the interface trigger the water molecules to group in to tetrahedral structure.

The dissolution of sII hydrate cannot be solely diffusion controlled process as there is effect due to the crystal structure. There are many factors which are influencing the dissolution of sII mixed hydrate, and it is a collective phenomenon which just cannot be described based on filled or empty cavities, solubility of hydrate forming component.

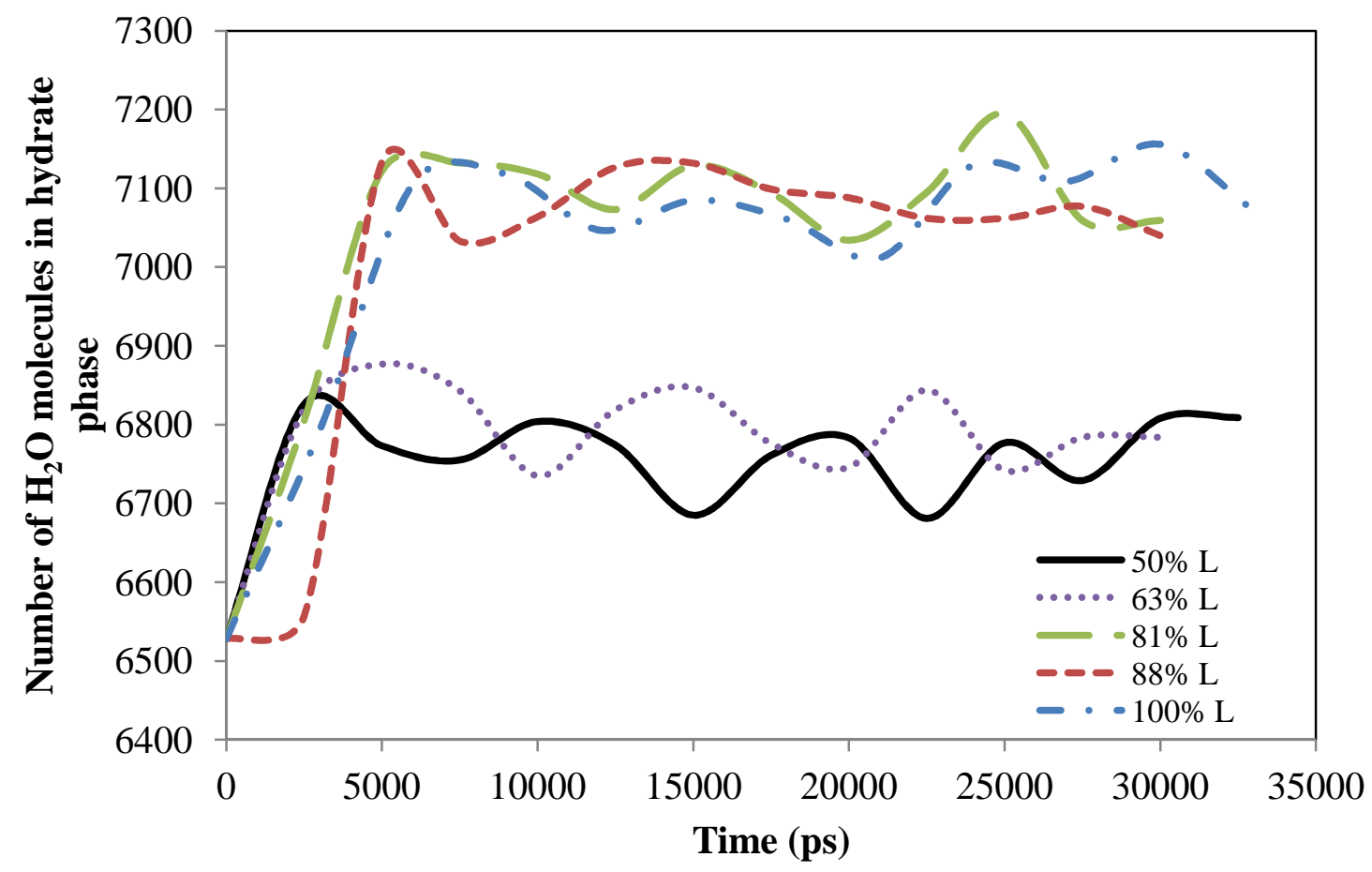

Figure 3.21 The number of water molecules in the hydrate phase during the course of the simulations. The small cage is $100 \%$ occupied. L-denotes large cages 


\subsection{Conclusions}

Molecular dynamics simulations were used to study the dissolution of the structure II methane-ethane-propane mixed hydrate in contact with the water layer. Simulations were performed by varying the composition of the guest hydrocarbons in the small and large cages. The intermolecular potentials used were verified by calculating the lattice constant of sII hydrate which is in agreement with the experimental value. In all the simulations performed there were no ethane or propane molecules which moved in to the bulk water phase from the hydrate phase, only the methane molecules dissolved in to the liquid water phase. The fully occupied hydrate was found to be more stable than the presence of empty cages. The number of methane molecules which moved in to the liquid phase was found to be increasing with decrease in the cage occupancy for the large cage. No affect was found on the small cage occupancy in the dissolution of methane, indicating that the small cages are more stable and the empty small cages do not destabilize the sII hydrate structure. Most of the methane molecules which moved in to the liquid water phase have come from the large cages. The dissolution of sII hydrate is linear in first few nanoseconds of the simulations and after that the dissolution behavior demonstrates an oscillatory behavior. The oscillatory behavior was due to the hydrate formation and breakup of the cages at the interface. There was no particular trend was observed on the dissolution of sII hydrate with the composition of the ethane and propane in the large cage, but the presence of these higher hydrocarbons reduces the dissolution of sII hydrate. Also, the amount of dissolution in sI methane hydrates is more than the sII hydrate for different cage occupancies, indicating that the higher hydrocarbons in the sII hydrate stabilizes the hydrate and reduces the dissolution. So from the above observations the hydrate dissolution of sII hydrate is the combination of many factors and cannot be solely diffusion controlled process. 


\subsection{A Thermodynamic Model to Predict the Hydrocarbon Solubility in Water at Two Phase Hydrate-Liquid Water Equilibrium}

In the chapter 3 , the hydrocarbon solubility values were used to calculate the maximum number of guest hydrocarbons can be dissolved in to the liquid water phase. In this chapter it will provide the details how those solubilities of hydrocarbons at two phase $\mathrm{H}-\mathrm{L}_{\mathrm{w}}$ values were obtained.

\subsection{Introduction}

Hydrates can form from a single phase system, in the absence of vapor phase, consisting of liquid water with dissolved hydrate former under appropriate temperature, pressure and dissolved hydrate former content ${ }^{69,75,85}$. The most probable conditions for the two-phase hydrate and water equilibrium can be found typically in the sediments of the marine environments with pressures ranging from 8 to $60 \mathrm{MPa}$ and temperatures ranging from $275 \mathrm{~K}$ to $293 \mathrm{~K}$. A better knowledge of hydrate-liquid water $\left(\mathrm{H}-\mathrm{L}_{\mathrm{w}}\right)$ two phase equilibrium is necessary for studying the formation and accumulation of subsea hydrates ${ }^{63}$, modeling the dissolution rates of hydrates in under-saturated surrounding environment ${ }^{33,64}$. The driving force for gas bubble dissolution with/without hydrate is the concentration difference between the bulk gas concentration and the gas solubility at the interface. Thus, the understanding of gas solubility is essential for modeling the dissolution rate. The hydrate former solubility in pure water dependency on temperature and pressure in the liquidwater equilibrium region $\left(\mathrm{L}_{\mathrm{w}}-\mathrm{V}\right)$ are different from the $\mathrm{H}-\mathrm{L}_{\mathrm{w}}$ equilibrium region at the corresponding temperature and pressure ${ }^{65-66}$. The solubility of hydrate former gas in water in $\mathrm{L}_{\mathrm{w}}-\mathrm{V}$ equilibrium decreases with increase in temperature at a given pressure, while in $\mathrm{H}-\mathrm{L}_{\mathrm{w}}$ region the solubility in pure water increases with increase in temperature ${ }^{66-69}$. 
Most of the available experimental data on hydrate equilibrium is on three phase $\mathrm{H}-\mathrm{L}_{\mathrm{w}}-\mathrm{V}$ equilibrium and the experimental data on the two phase $\mathrm{H}-\mathrm{L}_{\mathrm{w}}$ equilibrium is scarce. Also, these $\mathrm{H}$ $\mathrm{L}_{\mathrm{w}}$ experimental data refers to pure component methane, carbon dioxide, ethane and propane ${ }^{66-}$ ${ }^{69,74,134}$. To our knowledge there is no reliable experimental data on the solubility of hydrocarbon mixture (methane-ethane-propane) components in the aqueous phase at $\mathrm{H}-\mathrm{L}_{\mathrm{w}}$ equilibrium. There have been also some attempts to develop a model to predict the solubility of hydrate forming gas in the $H-L_{w}$ equilibrium, but these models are for estimating the solubility of pure hydrocarbon gas in water. $^{65,67,75-77}$

Hashemi et al. ${ }^{65}$ developed a model for the calculation of methane solubility in water at $\mathrm{H}$ $\mathrm{L}_{\mathrm{w}}$ equilibrium based on the van der Waals and Platteeuw model. They have used the TrebbleBishnoi equation of state for fugacity calculation. In their model, they have to readjust the reference chemical potential and reference enthalpy difference parameters with three phase vapor-liquid water-hydrate $\left(\mathrm{VL}_{\mathrm{w}} \mathrm{H}\right)$ equilibrium experimental data to improve the accuracy of the model. It was shown for the $\mathrm{VL}_{\mathrm{w}} \mathrm{H}$ phase equilibrium calculations that when the parameters are fitted to the experimental data the model works well in the range of the fit, but fail when extended outside the range of fit and also for mixed hydrates ${ }^{78}$. Mohammadi and Richon ${ }^{77}$ model for $\mathrm{H}-\mathrm{L}_{\mathrm{w}}$ equilibrium used the Langmuir constant values from the equation of Parrish and Praustniz ${ }^{79}$ for the evaluation of the fugacity of water in the hydrate phase based on the van der Waals and Platteeuw model. The Langmuir constant, which accounts for the guest-host interactions, is an important term in the van der Waals and Platteeuw model. Over the years, there has been a lot of improvement in the Langmuir constant calculation: including guest-host interactions beyond the first shell, evaluation of full configurational integral and more realistic guest-host intermolecular potentials ${ }^{80-82}$. Parrish and Praustniz used the spherical core Kihara-type potential to describe the interactions between the 
guest and the surrounding water molecules, these Kihara potential parameters were obtained by empirical fit to hydrate equilibrium pressures. However, these parameters set of Parrish and Praustniz failed to accurately predict equilibrium pressures for different gas mixtures and temperature ${ }^{82-84}$. These Langmuir constants used by Mohammadi and Richon ${ }^{77}$ in their model for $\mathrm{H}-\mathrm{L}_{\mathrm{w}}$ equilibrium calculations may attribute for the deviation to the experimental data. Sun and Duan $^{76}$ predicted the solubility of methane in water at $\mathrm{H}-\mathrm{L}_{\mathrm{w}}$ equilibrium using the van der Waals and Platteeuw model and angle dependent ab initio intermolecular potentials. Their model prediction of methane solubility is as good as our model, but they did not predict for ethane and propane and also for the mixed hydrates in the $\mathrm{H}-\mathrm{L}_{\mathrm{w}}$ region. There are also some other models for $\mathrm{H}-\mathrm{L}_{\mathrm{w}}$ equilibrium ${ }^{69-70,85}$, but these models require considerable efforts to fit experimental data. Naturally occurring hydrates are not pure simple hydrates, they form mixed hydrates with the presences of higher hydrocarbons. Accurate knowledge of solubility of hydrocarbon mixture in water in the hydrate stability zone (HSZ) plays an important role to understand the stability of gas hydrates..$^{30,33}$

The objective of this work is to develop an accurate model to determine the solubility of pure hydrocarbons and methane-ethane-propane mixture in water, when in equilibrium with gas hydrates $\left(\mathrm{L}_{\mathrm{w}}-\mathrm{H}\right)$. van der Waals and Platteeuw ${ }^{92}$ and Holder et al. ${ }^{95}$ models were employed to calculate the chemical potential of water in hydrate phase and liquid phase respectively. The fugacity of hydrate former hydrocarbon in the liquid water phase was calculated using the Poynting correction approach ${ }^{75}$. The Langmuir constant, which is the key term in the van der Waals and Platteeuw model, was obtained from the cell potential parameters. We have used different reference chemical potential difference parameters and reference enthalpy difference parameters for each guest molecule and for mixture the mixing rules were used to calculate the reference parameters. 
The model predictions are compared with the available experimental data for pure methane, ethane and propane guest molecules solubility at $\mathrm{H}-\mathrm{L}_{\mathrm{w}}$ equilibrium. The solubility of methane-ethanepropane mixture in water at $\mathrm{H}-\mathrm{L}_{\mathrm{w}}$ equilibrium is also predicted with this model.

\subsection{Thermodynamic Model}

The statistical thermodynamics model proposed by van der Waals and Platteeuw ${ }^{92}$, with the knowledge of crystal structure of hydrates, for the difference in chemical potential between the empty lattice $\left(\mu_{w}{ }^{\beta}\right)$ and fully filled hydrate lattice is given as in equation 1 .

$$
\Delta \mu_{w}^{\beta-H}=-R T \sum_{j=1}^{2} v_{j} \ln \left(1-\sum_{i} \theta_{j i}\right)
$$

where $v_{j}$ is the number of $j$-types cavities per water molecule, $\theta_{j i}$ is the fractional occupancy of $j$ type cavities which are occupied with $i$-type guest molecules. The cage occupancy, $\theta_{j i}$, is given as in equation 2.

$$
\theta_{j i}=\frac{C_{j i} f_{i}}{1+\sum_{i} C_{j i} f_{i}}
$$

where $f_{i}$ is the fugacity of hydrate forming species $i$ in the phase with which the hydrate phase is in equilibrium. $C_{j i}$ is the temperature-dependent Langmuir constant for species $i$ in cavity $j$ defined for non -linear and non-spherical guest molecule as,

$$
C_{j i}=\frac{Z_{j i}}{k T}=\frac{1}{8 \pi^{2} k T} \int_{V} \exp \left(-\frac{\Phi(r, \theta, \phi, \alpha, \beta, \gamma)}{k T}\right) r^{2} \sin \theta d r d \theta d \phi d \alpha \sin \beta d \beta d \gamma
$$

$Z_{j i}$ is the configurational integral, which depends on the total interaction potential $\Phi=\sum \Phi_{\mathrm{ij}}$ between the guest molecule and the host molecules surrounding it ${ }^{80,93} . Z_{j i}$ is a function of the polar coordinates $r, \theta, \phi$ of the guest molecule and $\alpha, \beta, \gamma$, the Euler angles that describes the orientation of guest molecule. 
The method for predicting equilibrium is based on the criterion that the chemical potentials $(\mu)$ of each component in the coexisting phases are equal. So at equilibrium,

$$
\mu_{w}{ }^{H}(T, P)=\mu_{w}{ }^{L}(T, P)
$$

where $\mu_{w}{ }^{H}(T, P)$ is the chemical potential of water $(w)$ in the hydrate phase $(H)$, and $\mu_{w}{ }^{L}(T, P)$ is the chemical potential of water in the water rich $(L)$ at a given temperature, $T$, and pressure, $P$. Using $\mu_{w}{ }^{\beta}$, the chemical potential of hypothetical empty hydrate lattice, the condition for equilibrium can be written as in Equation 13.

$$
\Delta \mu_{w}^{\beta-H}=\Delta \mu_{w}^{\beta-L}
$$

where $\quad \Delta \mu_{w}{ }^{\beta-H} \equiv \mu_{w}{ }^{\beta}-\mu_{w}{ }^{H}, \quad \Delta \mu_{w}{ }^{\beta-L} \equiv \mu_{w}{ }^{\beta}-\mu_{w}{ }^{L}$

The chemical potential difference, $\Delta \mu_{w}{ }^{\beta-L}$, between the hypothetical empty hydrate lattice and water in the hydrate phase is given by Holder et al. ${ }^{95}$ as

$$
\frac{\Delta \mu_{w}^{\beta-L}(T, P)}{k T}=\frac{\Delta \mu_{w}^{0}\left(T_{0}, 0\right)}{k T}-\int_{T_{o}}^{T} \frac{\Delta H_{w}^{\beta-L}}{k T^{2}} d T+\int_{o}^{P} \frac{\Delta V_{w}^{\beta-L, \alpha}}{k T} d P-\ln a_{w}
$$

where $\Delta \mu_{w}{ }^{0}\left(T_{0}, 0\right)$ is the reference chemical potential difference at the reference temperature, $T_{0}$, and zero pressure. The reference temperature, $T_{0}$, is usually taken as $273.15 \mathrm{~K}$. The last term in Equation 6 is activity of water, $a_{w}$, and is defined as

$$
a_{w}=\frac{f_{w}{ }^{L}}{f_{w}}
$$

where $f_{w}{ }^{L}$ is the fugacity of water in the water rich aqueous phase and $f_{w}$ is the water fugacity at the reference state, the pure water phase. The temperature-dependent enthalpy difference is given by Equation 8 .

$$
\Delta H_{w}{ }^{\beta-L}=\Delta H_{w}{ }^{0}\left(T_{0}\right)+\Delta H_{w}{ }^{L-\alpha}+\int_{T_{0}}^{T} \Delta C_{P} d T
$$


where $\Delta H_{w}{ }^{0}\left(T_{0}\right)$ is the reference enthalpy difference between the empty hydrate lattice and the pure water phase at reference temperature $T_{0}$.

The reference chemical potential difference, $\Delta \mu_{w}{ }^{0}$, vary with the size of the guest molecule and are different for each guest molecule, as there is a distortion in the lattice when the size of the guest molecule is increased $^{103,135}$. Variable reference parameters were used to account for lattice distortion instead of using same reference parameters corresponding to the hydrate structure for all the guests. In our group, a correlation was developed for the reference parameters, $\Delta \mu^{0}{ }_{w}, \Delta H^{0}{ }_{w}$, for each guest which are similar to the functional form of Lee and Holder ${ }^{136}$. These correlations are a function of molecular diameter of gas, $d_{g}$. The parameters of the correlations were obtained by Garapati ${ }^{137-138}$ using the regression analysis of the ab initio calculated reference parameters, $\Delta \mu^{0}{ }_{w}$, $\Delta H^{0}{ }_{w}$, of $\mathrm{CO}_{2}{ }^{100,139}$ and $\mathrm{CH}_{4}{ }^{93}$ for sI hydrate and $\mathrm{Ar}^{93}$ and $\mathrm{N}_{2}$ for sII hydrate. The $\Delta \mu^{0}{ }_{w}, \Delta H^{0}{ }_{w}$ based on the guest size $\left(d_{g}\right.$ in $\AA$ ) is given in Equation 9 and 10 for sI and in Equation 11 and 12 for sII respectively.

Structure I

$$
\begin{gathered}
\Delta \mu^{0}{ }_{w}=1197.279 \exp \left(0.0010933 * d_{g}\right) \\
\Delta H^{0}{ }_{w}=1061.589 \exp \left(0.0010933 * d_{g}\right)
\end{gathered}
$$

Structure II

$$
\begin{gathered}
\Delta \mu^{0}{ }_{w}=974.033 \exp \left(0.026446 * d_{g}\right) \\
\Delta H^{0}{ }_{w}=1044.658 \exp \left(0.056329 * d_{g}\right)
\end{gathered}
$$

The reference chemical potential difference and reference enthalpy difference values used in this work are given in Table 4. The reference parameters for mixed hydrates are calculated from the pure components using the following mixing rules:

$$
\Delta \mu^{0}{ }_{w, m i x}=\sum_{i}^{N} \Delta \mu_{w, i}^{0} z_{i}
$$




$$
\Delta H^{0}{ }_{w, m i x}=\sum_{i}^{N} \Delta H^{0}{ }_{w, i} z_{i}
$$

where $N$ is the number of guest components and $z_{i}$ is the hydrocarbon composition of the $i^{t h}$ component in hydrate phase on water free basis.

The heat capacity difference between the empty hydrate lattice and the pure water phase, $\Delta C_{P}$, is also temperature dependent and it is approximated by the following expression

$$
\Delta C_{P}=\Delta C_{P}^{0}\left(T_{0}\right)+b\left(T-T_{0}\right)
$$

where, $\Delta C_{P}{ }^{0}\left(T_{0}\right)$ is the reference heat capacity difference at the reference temperature $T_{0}$. The constant, $b$, represents the dependence of heat capacity on the temperature. The volume difference, $\Delta V_{w}{ }^{\beta-L}$, is assumed to be constant. The reference parameters used in this work are given in Table 4.1 .

\begin{tabular}{|c|c|c|c|c|c|}
\hline & Structure I & & Structure II & & Source \\
\hline Component & $\Delta \mu_{w}{ }^{0}(\mathrm{~J} / \mathrm{mol})$ & $\Delta H_{w}{ }^{0}(\mathrm{~J} / \mathrm{mol})$ & $\Delta \mu_{w}{ }^{0}(\mathrm{~J} / \mathrm{mol})$ & $\Delta H_{w}{ }^{0}(\mathrm{~J} / \mathrm{mol})$ & \\
\hline Methane & 1203.00 & 1170.00 & 1093.07 & 1337.89 & 93 \\
\hline Ethane & 1204.50 & 1195.59 & 1126.52 & 1431.90 & \\
\hline Propane & - & - & 1150.00 & 1488.00 & \\
\hline$\Delta \mathrm{V}_{w}^{\beta-\alpha}\left(\mathrm{m}^{3} / \mathrm{mol}\right)$ & \multicolumn{2}{|c|}{$3.0 \times 10^{-6}$} & \multicolumn{2}{|c|}{$3.4 \times 10^{-6}$} & 94 \\
\hline$\Delta \mathrm{V}_{w}{ }^{L-\alpha}\left(\mathrm{m}^{3} / \mathrm{mol}\right)$ & \multicolumn{4}{|c|}{$-1.598 \times 10^{-6}$} & \\
\hline$\Delta H_{w}{ }^{L-\alpha}(\mathrm{J} / \mathrm{mol})$ & \multicolumn{4}{|c|}{6009.5} & \\
\hline$\Delta C_{p}^{\beta-L}(\mathrm{~J} / \mathrm{mol} . \mathrm{K})$ & \multicolumn{4}{|c|}{$-37.32+0.179\left(T-T_{0}\right)$} & 95 \\
\hline
\end{tabular}

Table 4.1 The values of reference properties for structure I and structure II hydrates 


\subsubsection{Langmuir constant calculation}

The important term in the van der Waals and Platteeuw statistical thermodynamic model is the Langmuir constant. The Langmuir constant depends on the total interaction potential between the host water molecules and the guest hydrocarbon. Most of the previous models for hydrates calculated the Langmuir constant from the Kihara potential model with parameters arbitrary adjusting to the experimental phase equilibrium data. These empirically fitted potentials are aphysical and fundamentally not based on the guest host interactions. Also, these fitted parameters works well in the experimental range but fail when extended outside the range of fit, including when applied to mixed hydrate ${ }^{78}$. A full configurational integral has to be evaluated to accurately represent the total interaction potential ${ }^{80}$. Anderson et al. ${ }^{93}$ calculated the Langmuir constants from the site-site $a b$ initio intermolecular potentials by calculating the full six dimensional integral for methane gas hydrates over 5 hydrate shells. The resulting Langmuir constants computed from the $a b$ initio data are fitted to the van't off temperature dependence functional form ${ }^{78}$, given by Equation 4.16.

$$
C_{j i}(\beta)=C_{0 j i} \exp \left(m_{j i} \beta\right)
$$

where, $\beta=1 / k T, C_{0 j i}$ and $m_{j i}$ are cell potential parameters and are specific to the guest molecule $i$, and the cavity $j$ occupied. Hydrates that occupy only the large cages, the cell potential parameters can be obtained directly from the experimental dissociation data ${ }^{78,97,126}$ using the variable reference parameters. The cell potential parameters used in this work for the guest molecules are given in Table 4.2 and Table 4.3 for sI and sII hydrates respectively. 
Table 4.2 Cell potential parameters for structure I hydrate

\begin{tabular}{ccccc}
\hline & Small Cage & \multicolumn{3}{c}{ Large Cage } \\
\hline Component & $\ln C_{0}$ & $m$ & $\ln C_{0}$ & $m$ \\
Methane & -11.6900 & 5.6454 & -10.2200 & 5.6650 \\
Ethane & - & - & -12.5402 & 8.2236 \\
Propane & - & - & - & - \\
\hline
\end{tabular}

Table 4.3 Cell potential parameters for structure II hydrates

\begin{tabular}{ccccc}
\hline & \multicolumn{2}{c}{ Small Cage } & \multicolumn{2}{c}{ Large Cage } \\
\hline Component & $\ln C_{0}$ & $m$ & $\ln C_{0}$ & $m$ \\
Methane & -11.689 & 5.5140 & -8.6930 & 4.9616 \\
Ethane & - & - & -10.9615 & 8.9579 \\
Propane & - & - & -14.8015 & 12.4594 \\
\hline
\end{tabular}

\subsubsection{Fugacity of hydrocarbon in aqueous phase}

At hydrate-liquid water-vapor $\left(\mathrm{HL}_{\mathrm{w}} \mathrm{V}\right)$ three phase equilibrium, the fugacity of the hydrate

forming species, $f_{i}$, in the hydrate phase $\left(f_{i}^{H}\right)$ is equal to that in vapor phase $\left(f_{i}^{V}\right)$ and in liquid phase $\left(f_{i}^{L}\right)$. The fugacity of the pure gas or gas mixture $\left(f_{i}^{V}\right)$ can be calculated from a PVTN equation of state (EOS). We have used Peng-Robinson ${ }^{140}$ EOS for the vapor fugacity coefficient calculations. In the case of $\mathrm{H}-\mathrm{L}_{\mathrm{w}}$ equilibrium where there is no vapor phase, the fugacity of the hydrate forming species, $f_{i}$, in the hydrate phase is equal to that in liquid water phase. The fugacity of hydrocarbon in the single liquid phase is calculated using the thermodynamic method described by Holder et al. ${ }^{75}$ as

$$
f_{i}^{L}\left(T, P, x_{i}\right)=f_{i}^{s a t} \gamma_{i} \exp \left[\int_{P}^{P}{ }^{s a t} \frac{\bar{V}_{l} d p}{R T}\right]
$$


where $\bar{V}_{l}$ is the partial molar volume of species $i$ in the water phase, $\gamma_{i}$ is the activity coefficient of hydrocarbon and is unit for pure water. The activity coefficients are not unit for the presence of electrolytes in water. $P^{s a t}$ is the pressure required to obtain a given solubility $x_{i}$ and $f_{i}^{\text {sat }}$ is the fugacity of hydrocarbon at $P^{s a t}$. A common assumption is that $\bar{V}_{l}$ is constant, and Equation 4.17 can be modified as

$$
f_{i}^{L}\left(T, P, x_{i}\right)=f_{i}^{s a t} \gamma_{i} \exp \left[\frac{\bar{V}_{l}\left(P-P^{s a t}\right)}{R T}\right]
$$

The partial molar volumes $\left(\bar{V}_{l}\right)$ of the hydrocarbon at $298 \mathrm{~K}$ are given in Table 4.4. The $\bar{V}_{l}$ are corrected for temperature using the following approximation ${ }^{141}$ given in Equation 4.19. $V_{w}{ }^{0}$ is the molar volume of water at standard state (saturation).

$$
\bar{V}_{l}(T)=\bar{V}_{l}(298 K) \frac{V_{w}^{0}(T)}{V_{w}^{0}(298 K)}
$$

Henry's law is used to calculate the solubility of hydrocarbon in water, and the Equation 4.18 is modified as

$$
f_{i}^{L}\left(T, P, x_{i}\right)=x_{i} H_{i w} \gamma_{i} \exp \left[\frac{\bar{V}_{l}\left(P-P^{s a t}\right)}{R T}\right]
$$

At $P^{s a t}$, the hydrocarbon rich vapor phase coexist with water rich liquid phase $\left(\mathrm{V}-\mathrm{L}_{\mathrm{w}}\right.$ phase boundary). The fugacity's at V- $\mathrm{L}_{\mathrm{w}}$ equilibrium are calculated using the Peng-Robinson equation of state $^{142}$ for hydrocarbon rich phase and by Henry's constant for the liquid water phase at a given solubility $x_{i}$. The Henry's constants $\left(H_{i w}\right)$ for methane ${ }^{141}$, ethane ${ }^{143}$ and propane ${ }^{144}$ are given in Equations, 4.21, 4.22 and 4.23 respectively.

$$
\begin{gathered}
H_{i w}=\exp \left(5.135+\frac{7837}{T}-\frac{1.5090 \times 10^{6}}{T^{2}} \frac{2.06 \times 10^{7}}{T^{3}}\right) \times 0.01 \\
H_{i w}=10^{146.901-\frac{5768.3}{T}-51.8593 \log (T)+0.01741 \times T} \\
H_{i w}=\exp \left(552.64799+0.078453 \times T-\frac{21334.4}{T}-85.89736 \ln (T)\right) \times 0.01
\end{gathered}
$$


where $T$ is in Kelvin and $H_{i w}$ is given in bars.

Table 4.4 Partial molar volume of hydrocarbons

\begin{tabular}{|c|c|}
\hline Component & $\bar{V}_{l}(298 \mathrm{~K})\left(\mathrm{m}^{3} / \mathrm{Kmol}\right)$ \\
\hline Methane $^{145}$ & 0.0398 \\
\hline Ethane $^{146}$ & 0.0530 \\
\hline Propane $^{146}$ & 0.0710 \\
\hline
\end{tabular}

\subsubsection{Activity of water and activity coefficients in seawater}

The solubility of methane, ethane and propane in water is less so we assume that the activity of water $\left(a_{w}\right)$ will not change with these sparingly soluble gases and is taken as unity for pure water. However, the presence of electrolytes in liquid water will change the activity of water and the aqueous hydrocarbons solubility. The presence of electrolytes will not change the chemical potential of water in the hydrate phase as they cannot enter the hydrate lattice, but the chemical potential of liquid water is changed as the activity of water is changed affecting the hydrate thermodynamic equilibrium. The activity of water is modeled using the Pitzer ${ }^{147}$ equation in this study. The activity of water $\left(a_{w}\right)$ is given as

$$
\ln a_{w}=-\phi M_{H_{2} O} \sum_{i} m_{i}
$$

where $m_{i}$ is the molality $(\mathrm{mol} / \mathrm{kg})$ of ions, $M_{\mathrm{H}_{2} \mathrm{O}}$ is the molecular weight of water and $\phi$ is the osmotic coefficient and is given as

$$
\begin{gathered}
\sum m_{i}(\phi-1)=2\left[\frac{-A^{\phi} I^{1.5}}{1+1.2 I^{0.5}}+\sum_{c} \sum_{a} m_{c} m_{a}\left(B_{c a}{ }^{\phi}+Z C_{c a}\right)+\sum_{c<} \sum_{c^{\prime}} m_{c} m_{c^{\prime}}\left(\Phi_{c c^{\prime}}^{\phi}+\right.\right. \\
\left.\left.\sum_{a} m_{a} \Psi_{c c^{\prime} a}\right)+\sum_{a<} \sum_{a^{\prime}} m_{a} m_{a^{\prime}}\left(\Phi_{a a^{\prime}}^{\phi}+\sum_{c} m_{c} \Psi_{a a^{\prime} c}\right)\right]
\end{gathered}
$$


where $I$ is the ionic strength, the subscripts $c$ and $a$ represent cation and anion respectively. We have ignored the neutral-neutral atom interactions and neutral-electrolyte interaction contributions in the activity of water calculations, as they do not contribute much to the activity of water. $A^{\phi}$ is the Debye-Hückel limiting slope. $B$ and $\Phi$ are measurable combinations of the second virial coefficients. $C$ and $\Psi$ are measurable combinations of the third varial coefficients. The interaction parameters, the binary and tertiary parameters were determined by Spencer et al. ${ }^{148}$ as a temperature dependent and are given in Table 4.5 and 4.6. The effect of pressure on the activity of water is small so it can be ignored ${ }^{149}$.

$$
A^{\phi}=\frac{1}{3}\left[\frac{2 \pi N_{0} \rho_{w}}{1000}\right]\left(e^{2} / D k T\right)^{3 / 2}
$$

The ionic strength, $I$, is defined as

$$
I=1 / 2 \sum_{i} m_{i} z_{i}^{2}
$$

The function $\mathrm{Z}$ in Equation 4.25 is defined as

$$
Z=\sum_{i} m_{i}\left|z_{i}\right|
$$

The ionic strength dependence of the second virial coefficients, $B_{M X}{ }^{\phi}$, is defined as

$$
B_{M X}{ }^{\phi}=\beta^{0}{ }_{M X}+\beta^{1}{ }_{M X} e^{-\alpha_{M X} \sqrt{I}}
$$

when either cation $\mathrm{M}$ or anion $\mathrm{X}$ is univalent then $\alpha_{M X}=2$. For 2-2 or higher valence pair $\alpha_{M X}=$ 1.4. The third virial electrolyte, $C_{M X}$, is defined as

$$
C_{M X}=\frac{C^{\phi}{ }_{M X}}{2 \sqrt{\left|z_{M} z_{X}\right|}}
$$

The second virial coefficient, $\Phi$, which accounts for the interactions of like atoms are defined as

$$
\Phi^{\phi}{ }_{i j}=\theta_{i j}+{ }^{E} \theta_{i j}(I)+I^{E} \theta_{i j}(I)
$$

The interaction parameters, $\beta^{0}{ }_{M X}, \beta^{1}{ }_{M X}, C^{\phi}{ }_{M X}$ for each cation-anion pair and $\Psi_{a a^{\prime} c}, \theta_{i j}, \Psi_{c c^{\prime} a}$ are represented as a temperature dependent with the following empirical equation. 


$$
\operatorname{Par}(T)=a_{1}+a_{2} T+\frac{a_{3}}{T}+a_{4} \ln T+a_{5} T^{2}+a_{6} T^{3}
$$

Table 4.5 The temperature dependence of the Debye-huckel parameters and for the binary interaction parameters of Pitzer model ${ }^{147}$ determined by Spencer et al. $(1990)^{148}$

\begin{tabular}{|c|c|c|c|c|c|c|}
\hline & $a_{1}$ & $a_{2}$ & $a_{3}$ & $a_{4}$ & $a_{5}$ & $a_{6}$ \\
\hline $\mathrm{A}^{\phi}$ & $8.66836498 \mathrm{E}+01$ & 8.48795942E-02 & $-1.32731477 \mathrm{E}+03$ & $-1.76460172 \mathrm{E}+01$ & $-8.88785150 \mathrm{E}-05$ & 4.88096393E-08 \\
\hline \multicolumn{7}{|c|}{$\mathrm{Na}, \mathrm{Cl}$} \\
\hline$\beta^{(0)}$ & $7.87239712 \mathrm{E}+00$ & $-8.38640960 \mathrm{E}-03$ & $-4.96920671 \mathrm{E}+02$ & $-8.20972560 \mathrm{E}-01$ & $1.44137740 \mathrm{E}-05$ & $-8.78203010 \mathrm{E}-09$ \\
\hline$\beta^{(1)}$ & $8.66915291 \mathrm{E}+02$ & $6.06166931 \mathrm{E}-01$ & $-1.70460145 \mathrm{E}+04$ & $-1.67171296 \mathrm{E}+02$ & $-4.80489210 \mathrm{E}-04$ & $1.88503857 \mathrm{E}-07$ \\
\hline$C^{\varnothing}$ & $1.70761824 \mathrm{E}+00$ & $2.32970177 \mathrm{E}-3$ & $-1.35583596 \mathrm{E}+00$ & $-3.87767714 \mathrm{E}-01$ & $-2.46665619 \mathrm{E}-06$ & $1.21543380 \mathrm{E}-09$ \\
\hline \multicolumn{7}{|c|}{$\mathrm{K}, \mathrm{Cl}$} \\
\hline$\beta^{(0)}$ & $2.65718766 \mathrm{E}+01$ & 9.92715099E-03 & $-7.55707220 \mathrm{E}+02$ & $-4.67300770 \mathrm{E}+00$ & $-3.62323330 \mathrm{E}-06$ & $-6.28427180 \mathrm{E}-11$ \\
\hline$\beta^{(1)}$ & $1.69742977 \mathrm{E}+03$ & $1.22270943 \mathrm{E}+00$ & $-3.28684422 \mathrm{E}+04$ & $-3.28813848 \mathrm{E}+02$ & $-9.99044490 \mathrm{E}-04$ & $4.04786721 \mathrm{E}-07$ \\
\hline$C^{\varnothing}$ & $-3.27571680 \mathrm{E}+00$ & $-1.27222054 \mathrm{E}-03$ & $9.07747666 \mathrm{E}+01$ & 5.80513562E-01 & 4.71374283E-07 & $1.11625070 \mathrm{E}-11$ \\
\hline \multicolumn{7}{|c|}{$\mathrm{Ca}, \mathrm{Cl}$} \\
\hline$\beta^{(0)}$ & $-5.62764702 \mathrm{E}+01$ & $-3.00771997 \mathrm{E}-02$ & $1.11730349 \mathrm{E}+03$ & $1.06664743 \mathrm{E}+01$ & $1.05630400 \mathrm{E}-05$ & $3.33316260 \mathrm{E}-09$ \\
\hline$\beta^{(1)}$ & $3.4787000 \mathrm{E}+00$ & $-1.54170000 \mathrm{E}-02$ & 0.0 & 0.0 & $3.17910000 \mathrm{E}-05$ & 0.0 \\
\hline$C^{\varnothing}$ & $2.64231655 \mathrm{E}+01$ & 2.46922993E-02 & $-4.18098427 \mathrm{E}+02$ & $-5.35350322 \mathrm{E}+00$ & $-2.48298510 \mathrm{E}-05$ & $1.22421864 \mathrm{E}-08$ \\
\hline \multicolumn{7}{|c|}{$\mathrm{Mg}, \mathrm{Cl}$} \\
\hline$\beta^{(0)}$ & $3.13852913 \mathrm{E}+02$ & 2.61769099E-01 & $-5.53133381 \mathrm{E}+03$ & $-6.21616862 \mathrm{E}+01$ & $-2.46268460 \mathrm{E}-04$ & $1.15764787 \mathrm{E}-07$ \\
\hline$\beta^{(1)}$ & $-3.18432525 \mathrm{E}+04$ & $-2.86710358 \mathrm{E}+01$ & $5.24032958 \mathrm{E}+05$ & $6.40770396 \mathrm{E}+03$ & $2.78892838 \mathrm{E}-02$ & $-1.32797050 \mathrm{E}-05$ \\
\hline$C^{\varnothing}$ & $5.95320000 \mathrm{E}-02$ & $-2.49949 \mathrm{E}-04$ & 0.0 & 0.0 & $2.41831000 \mathrm{E}-07$ & 0.0 \\
\hline \multicolumn{7}{|c|}{$\mathrm{Na}, \mathrm{SO}_{4}$} \\
\hline$\beta^{(0)}$ & $-3.32486330 \mathrm{E}+03$ & $-2.92973530 \mathrm{E}+00$ & $5.53958527 \mathrm{E}+04$ & $6.66660369 \mathrm{E}+10$ & $2.80243670 \mathrm{E}-03$ & $-1.31688300 \mathrm{E}-06$ \\
\hline$\beta^{(1)}$ & $-3.57406160 \mathrm{E}+03$ & $-3.00112060 \mathrm{E}+00$ & $6.09716482 \mathrm{E}+04$ & $7.11613120 \mathrm{E}+02$ & $2.73660950 \mathrm{E}-03$ & $-1.21917100 \mathrm{E}-06$ \\
\hline$C^{\varnothing}$ & $3.68520478 \mathrm{E}+02$ & $3.16243995 \mathrm{E}-01$ & $-6.22607913 E+11$ & $-7.35844094 \mathrm{E}+01$ & $-2.95372760 \mathrm{E}-04$ & $1.35491104 \mathrm{E}-07$ \\
\hline \multicolumn{7}{|c|}{$\mathrm{K}, \mathrm{SO}_{4}$} \\
\hline$\beta^{(0)}$ & 4.07908797E+01 & $8.26906675 \mathrm{E}-03$ & $-1.41842998 \mathrm{E}+03$ & $-6.74728848 \mathrm{E}+00$ & 0.0 & 0.0 \\
\hline$\beta^{(1)}$ & $-1.31669510 \mathrm{E}+01$ & 2.35793239E-02 & $2.06712594 \mathrm{E}+03$ & 0.0 & 0.0 & 0.0 \\
\hline$C^{\varnothing}$ & $-1.88000000 \mathrm{E}-02$ & 0.0 & 0.0 & 0.0 & 0.0 & 0.0 \\
\hline \multicolumn{7}{|c|}{$\mathrm{Ca}, \mathrm{SO}_{4}$} \\
\hline$\beta^{(0)}$ & $1.50000000 \mathrm{E}-01$ & 0.0 & 0.0 & 0.0 & 0.0 & 0.0 \\
\hline$\beta^{(1)}$ & $3.00000000 \mathrm{E}+00$ & 0.0 & 0.0 & 0.0 & 0.0 & 0.0 \\
\hline$\beta^{(2)}$ & $-1.29399287 \mathrm{E}+02$ & 4.00431027E-01 & 0.0 & 0.0 & 0.0 & 0.0 \\
\hline \multicolumn{7}{|c|}{$\mathrm{Mg}, \mathrm{SO}_{4}$} \\
\hline$\beta^{(0)}$ & $5.40078490 \mathrm{E}+03$ & $4.90576884 \mathrm{E}+00$ & $-8.80664146 \mathrm{E}+04$ & $-1.08839565 \mathrm{E}+03$ & $-4.80489750 \mathrm{E}-03$ & $2.31126994 \mathrm{E}-06$ \\
\hline$\beta^{(1)}$ & $2.78730869 \mathrm{E}+00$ & 4.30077440E-03 & 0.0 & 0.0 & 0.0 & 0.0 \\
\hline$C^{\emptyset}$ & $-5.88623653 \mathrm{E}+02$ & $-5.05522880 \mathrm{E}-01$ & $1.02002016 \mathrm{E}+04$ & $1.17303808 \mathrm{E}+02$ & 4.82776570E-04 & $-2.30298380 \mathrm{E}-07$ \\
\hline
\end{tabular}


Table 4.6 The temperature dependence of mixed electrolyte parameters of Pitzer model ${ }^{147}$ determined by Spencer et al. (1990) ${ }^{148}$

\begin{tabular}{|c|c|c|c|c|c|}
\hline & $a_{1}$ & $a_{2}$ & $a_{3}$ & $a_{4}$ & $a_{5}$ \\
\hline$\theta_{\mathrm{Na}, \mathrm{K}}$ & $-1.82266741 \mathrm{E}+01$ & $-3.69038470 \mathrm{E}-03$ & $6.12415011 \mathrm{E}+02$ & $3.02994981 \mathrm{E}+00$ & 0.0 \\
\hline$\Psi_{\mathrm{Na}, \mathrm{K}, \mathrm{Cl}}$ & $6.48108127 \mathrm{E}+00$ & $1.46803468 \mathrm{E}-03$ & $-2.04354019 \mathrm{E}+00$ & $-1.09448043 \mathrm{E}+00$ & 0.0 \\
\hline$\Psi_{\mathrm{Na}, \mathrm{K}, \mathrm{SO}_{4}}$ & $3.48120 \mathrm{E}-02$ & 0.0 & $-8.21660 \mathrm{E}+00$ & 0.0 & 0.0 \\
\hline$\theta_{\mathrm{Na}, \mathrm{Ca}}$ & $5.0 \mathrm{E}-02$ & 0.0 & 0.0 & 0.0 & 0.0 \\
\hline$\Psi_{\mathrm{Na}, \mathrm{Ca}, \mathrm{Cl}}$ & $-7.6398 \mathrm{E}+00$ & $-1.299 \mathrm{E}-02$ & 0.0 & $1.8475 \mathrm{E}+00$ & $1.1060 \mathrm{E}-05$ \\
\hline$\Psi_{\mathrm{Na}, \mathrm{Ca}, \mathrm{SO}_{4}}$ & $-1.20 \mathrm{E}-02$ & 0.0 & 0.0 & 0.0 & 0.0 \\
\hline$\theta_{\mathrm{Na}, \mathrm{Mg}}$ & 7.0E-02 & 0.0 & 0.0 & 0.0 & 0.0 \\
\hline$\Psi_{\mathrm{Na}, \mathrm{Mg}, \mathrm{Cl}}$ & $-3.10987 \mathrm{E}-02$ & $5.44647800 \mathrm{E}-05$ & $1.99404210 \mathrm{E}+00$ & 0.0 & 0.0 \\
\hline$\Psi_{\mathrm{Na}, \mathrm{Mg}, \mathrm{SO}_{4}}$ & $1.175052 \mathrm{E}-01$ & 0.0 & $-4.19862 \mathrm{E}+01$ & 0.0 & 0.0 \\
\hline$\theta_{\mathrm{K}, \mathrm{Ca}}$ & $2.36571 \mathrm{E}+00$ & $-4.54 \mathrm{E}-03$ & $-2.849400 \mathrm{E}+02$ & 0.0 & 0.0 \\
\hline$\Psi_{\mathrm{K}, \mathrm{Ca}, \mathrm{Cl}}$ & $-5.930602 \mathrm{E}+00$ & $2.54280 \mathrm{E}-04$ & $-1.34390 \mathrm{E}+01$ & 0.0 & 0.0 \\
\hline$\theta_{\mathrm{K}, \mathrm{Mg}}$ & $1.1670 \mathrm{E}-01$ & 0.0 & 0.0 & 0.0 & 0.0 \\
\hline$\Psi_{\mathrm{K}, \mathrm{Mg}, \mathrm{Cl}}$ & $5.0362230 \mathrm{E}-02$ & $-8.75082 \mathrm{E}-06$ & $-2.89909 \mathrm{E}+01$ & 0.0 & 0.0 \\
\hline$\Psi_{\mathrm{K}, \mathrm{Mg}, \mathrm{SO}_{4}}$ & $-1.36791570 \mathrm{E}+00$ & 4.24016653E-03 & 0.0 & 0.0 & 0.0 \\
\hline$\theta_{\mathrm{Ca}, \mathrm{Mg}}$ & $5.31274136 \mathrm{E}+00$ & -6.34242480 E-03 & $-9.83113847 \mathrm{E}+02$ & 0.0 & 0.0 \\
\hline$\Psi_{\mathrm{Ca}, \mathrm{Mg}, \mathrm{Cl}}$ & $4.1579022 \mathrm{E}+01$ & $1.30377312 \mathrm{E}-02$ & $-9.81658526 \mathrm{E}+02$ & $-7.4061986 \mathrm{E}+00$ & 0.0 \\
\hline$\theta_{\mathrm{Cl}_{,}, \mathrm{SO}_{4}}$ & 7.0E-02 & 0.0 & 0.0 & 0.0 & 0.0 \\
\hline$\Psi_{\mathrm{Cl}_{1}, \mathrm{SO}_{4}, \mathrm{Na}}$ & $4.0209775 \mathrm{E}+00$ & $1.1286005 \mathrm{E}-03$ & $-1.01169260 \mathrm{E}+02$ & $-7.060798 \mathrm{E}-01$ & 0.0 \\
\hline$\Psi_{\mathrm{Cl}_{2}, \mathrm{SO}_{4}, \mathrm{~K}}$ & $-2.124815 \mathrm{E}-01$ & $2.8469833 \mathrm{E}-04$ & $3.75619140 \mathrm{E}+00$ & 0.0 & 0.0 \\
\hline$\Psi_{\mathrm{Cl}_{1,}, \mathrm{SO}_{4}, \mathrm{Ca}}$ & $-1.8 \mathrm{E}-02$ & 0.0 & 0.0 & 0.0 & 0.0 \\
\hline$\Psi_{\mathrm{Cl}_{1}, \mathrm{SO}_{4}, \mathrm{Mg}}$ & $-1.839158 \mathrm{E}-01$ & $1.429444 \mathrm{E}-04$ & $3.2630 \mathrm{E}+01$ & 0.0 & 0.0 \\
\hline
\end{tabular}

The aqueous solubility of a nonelectrolyte is dependent on the concentration of and type of the salts present in solution. The activity coefficient of the neutral atom in electrolyte solution is calculated from Setschenow equation given as

$$
\ln \gamma_{i}=\sum_{j} k_{s} m_{j}
$$


where $k_{s}$ is the salting in or salting out coefficent. $m_{j}$ is the molality of the salt. The Setschenow coefficents for methane and ethane are determined by Millero ${ }^{150}$. The Setschenow coefficents are given in Table 4.7

Table 4.7 Salting coefficients for methane ${ }^{150}$, ethane ${ }^{150}$ and propane in electrolyte solution at $25^{\circ} \mathrm{C}$

\begin{tabular}{lcll}
\hline Salts & $k_{s}, \mathrm{CH}_{4}$ & $k_{s}, \mathrm{C}_{2} \mathrm{H}_{6}$ & $k_{s}, \mathrm{C}_{3} \mathrm{H}_{8}$ \\
\hline $\mathrm{NaCl}$ & 0.319 & 0.391 & 0.216 \\
$\mathrm{Na}_{2} \mathrm{SO}_{4}$ & 0.836 & 1.26 & \\
$\mathrm{KCl}$ & 0.233 & 0.375 & 0.121 \\
$\mathrm{NaHCO}_{3}$ & 0.468 & 0.586 & \\
$\mathrm{CaCl}_{2}$ & 0.497 & 0.723 & \\
$\mathrm{MgCl}_{2}$ & 0.435 & 0.659 & \\
\hline
\end{tabular}

\subsection{Results and Discussion}

The solubility of the hydrocarbon $\left(x_{i}\right)$ in water at $\mathrm{H}-\mathrm{L}_{\mathrm{w}}$ two phase equilibrium is calculated by solving Equation 4.5 for a given pressure and temperature by iterative procedure. First an initial guess is made for $x_{i}$, and the saturation pressure, $P^{\text {sat }}$, is calculated corresponding to $x_{i}$. Then we calculate the fugacity of the hydrocarbon, $f_{i}^{L}$, from Equation 4.20 and substitute to calculate the $\Delta \mu_{w}{ }^{\beta-H}$ from Equation 4.1 and 4.2. Finally the pressure is calculated using Equation 4.5 and 4.6 and compared it with the given pressure. If the absolute difference between the pressures is within the tolerance limit then $x_{i}$ is the solubility at that pressure and temperature, if not, $x_{i}$ is changed and the procedure is repeated. If equilibrium pressure has to be calculated at $\mathrm{H}-\mathrm{L}_{\mathrm{w}}$ two phase equilibrium for a given temperature and solubility, we guess an initial value for pressure. Then we calculate the fugacity of the hydrocarbon, $f_{i}^{L}$, from Equation 4.20 and substitute to calculate the 
$\Delta \mu_{w}{ }^{\beta-H}$ from Equation 4.1 and 4.2. Finally the pressure is calculated using Equation 4.5 and 4.6 and compares it with the guessed pressure. If the absolute difference between the pressures is within the tolerance limit then the pressure is the desired pressure, if not, the initial guess for pressure is changed and the procedure is repeated.

\subsubsection{H- $\mathrm{L}_{\mathrm{w}}$ Equilibrium for Pure Components}

There is not much of experimental data available in the literature for the solubility of hydrocarbon in water at $\mathrm{H}-\mathrm{L}_{\mathrm{w}}$ equilibrium. Among those reported for the solubility of methane in water at $\mathrm{H}-\mathrm{L}_{\mathrm{w}}$ equilibrium, the data by Lu et al. ${ }^{68}$, Servio and Englezos ${ }^{66}$, Kim et al. ${ }^{69}$ and Seo and Lee $^{71}$ are considered to be more reliable ${ }^{151}$. The other experimental data ${ }^{70,152}$ reported in the literature for methane solubility at $\mathrm{H}-\mathrm{L}_{\mathrm{w}}$ equilibrium are not used to evaluate the model accuracy in

this work, as they are found erroneous with other literature data ${ }^{68-69,151}$. The model predictions of the solubility of methane in water are compared to the four experimental data sets without using any adjustable parameters and are given in Table 4.8. The model predicts for methane solubility in two phase $\mathrm{H}-\mathrm{L}_{\mathrm{w}}$ equilibrium with an average absolute deviation (AAD) of $3.5 \%$ for Servio and Englezos $^{66}, 5.2 \%$ for Kim et al. ${ }^{69}, 6.8 \%$ for Lu et al. ${ }^{68}$ and $8.5 \%$ for Seo et al. ${ }^{71}$ The predicted results are in agreement to the experimental data. Most of the submarine hydrates located in the two phase region are with pressures ranging from 8 to the excess of $100 \mathrm{MPa}$. Lu et al ${ }^{68}$ measured the methane solubility data at $\mathrm{H}-\mathrm{L}_{\mathrm{w}}$ equilibrium using in situ Raman spectroscopy at high pressures. They gave an expression for methane solubility in equilibrium with hydrate as a function of pressure and temperature from the measured data. The solubility prediction by this model and by the expression given by Lu et al. ${ }^{68}$ are compared in Figure 4.1 up to the pressures of $100 \mathrm{MPa}$. As we can see from Figure 4.1, that the solubility of methane increases with increase in temperature 
and decreases with increase in pressure. Also, the effect of pressure on the solubility of methane in water in equilibrium with hydrate is small at a given temperature compare to the effect of temperature on the methane solubility at a given pressure.

Table 4.8 Experimental methane solubility data at H-Lw equilibrium

\begin{tabular}{ccccc}
\hline$T$ range (K) & $\begin{array}{c}P \text { range } \\
(\mathrm{MPa})\end{array}$ & $\begin{array}{c}\text { Number } \\
\text { of points }\end{array}$ & Reference & \% AAD \\
\hline $274.15-286.15$ & $6.0-20.0$ & 13 & 71 & 8.5 \\
$274.35-280.15$ & $3.5-6.5$ & 6 & 66 & 3.5 \\
$276.20-281.70$ & $5.0-14.4$ & 16 & 69 & 5.2 \\
$276.55-294.55$ & $10.0-40.0$ & 17 & 68 & 6.8 \\
\hline$\%$ AAD $=\frac{1}{N} \sum_{1}^{N}\left(\frac{\text { experimental-predicted }}{\text { experimental }}\right) \times 100$ & &
\end{tabular}

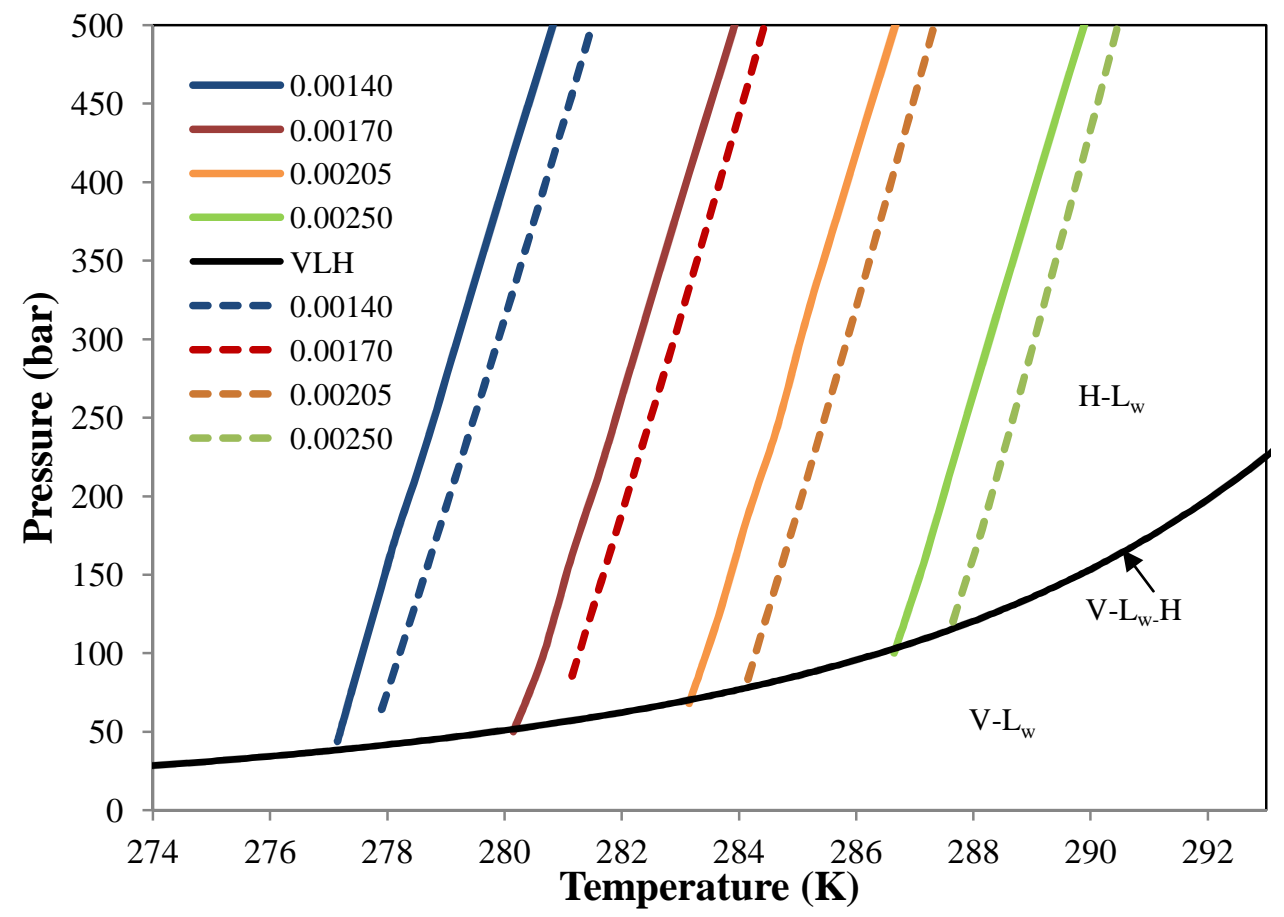

Figure 4.1 Comparison of mole fraction of methane in water at $\mathrm{H}-\mathrm{L}_{\mathrm{w}}$ equilibrium predicted by this model (solid lines) and by the expression given by Lu et al. ${ }^{68}$ (dotted lines). The bottom solid line is the VLH equilibrium for methane hydrate, plotted from the data obtained from Sloan ${ }^{134}$. 
Cage occupancy, the fraction of each cage occupied by the guest molecules, of hydrate at a temperature and pressure must be known to fully understand the thermodynamics of hydrate formation, decomposition and better predict the hydrate density. Methane hydrates form sI hydrate and occupies both small and large cages. Huo et al. ${ }^{153}$ and Seo et al. ${ }^{71}$ using Raman spectroscopy measured the cage occupancy ratio $\left(\theta_{L} / \theta_{S}\right)$ of large cage $\left(\theta_{L}\right)$ to small cage $\left(\theta_{S}\right)$ for methane hydrate at $\mathrm{H}-\mathrm{L}_{\mathrm{w}}$ equilibrium conditions. Cage occupancies can be calculated from Equation 2 for small cage and large cage at a temperature and pressure. Table 4.9 shows the cage occupancy ratio $\left(\theta_{L} / \theta_{S}\right)$ predicted by this model, experimental data and by Sun and Duan ${ }^{76}$ model. The cage occupancy of small cage and large cage increases with increase in temperature and pressure but the ratio, $\left(\theta_{L} / \theta_{S}\right)$, decreases with increase in temperature. This is similar to the trend predicted by Sun and Duan. ${ }^{76}$ Figure 4.2 shows the $\theta_{L} / \theta_{S}$ ratio effect on temperature at constant pressure and Figure 4.3 shows the small cage and large cage occupancy effect on temperature at constant pressure.

Table 4.9 Occupancy ratio, $\left(\boldsymbol{\theta}_{\boldsymbol{L}} / \boldsymbol{\theta}_{\boldsymbol{S}}\right)$, of methane sI hydrates at $\mathrm{H}-\mathrm{L}_{\mathrm{w}}$ equilibrium

\begin{tabular}{ccccc}
\hline $\begin{array}{c}\text { Temperature } \\
(\mathrm{K})\end{array}$ & $\begin{array}{c}\text { Pressure } \\
(\mathrm{MPa})\end{array}$ & $\begin{array}{c}\text { Experimental } \\
\text { value } \\
\left(\theta_{L} / \theta_{S}\right)\end{array}$ & $\begin{array}{c}\text { Sun and } \\
\text { Duan }^{76}\end{array}$ & $\begin{array}{c}\text { This } \\
\text { model }\end{array}$ \\
\hline 274.15 & 10 & 1.053 & 1.066 & 1.099 \\
275.15 & 30 & 1.167 & 1.050 & 1.078 \\
\hline
\end{tabular}




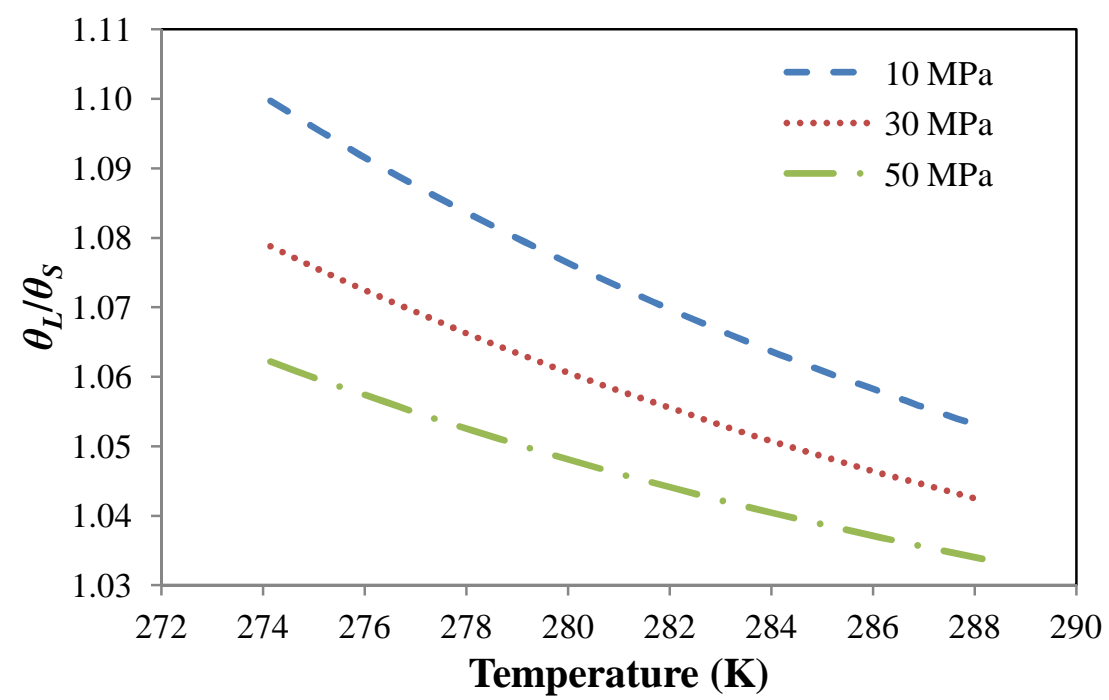

Figure 4.2 Cage occupancy ratio $\left(\boldsymbol{\theta}_{\boldsymbol{L}} / \boldsymbol{\theta}_{\boldsymbol{S}}\right)$ of sI methane hydrate at $\mathrm{H}-\mathrm{L}_{\mathrm{w}}$ equilibrium predicted by this model.

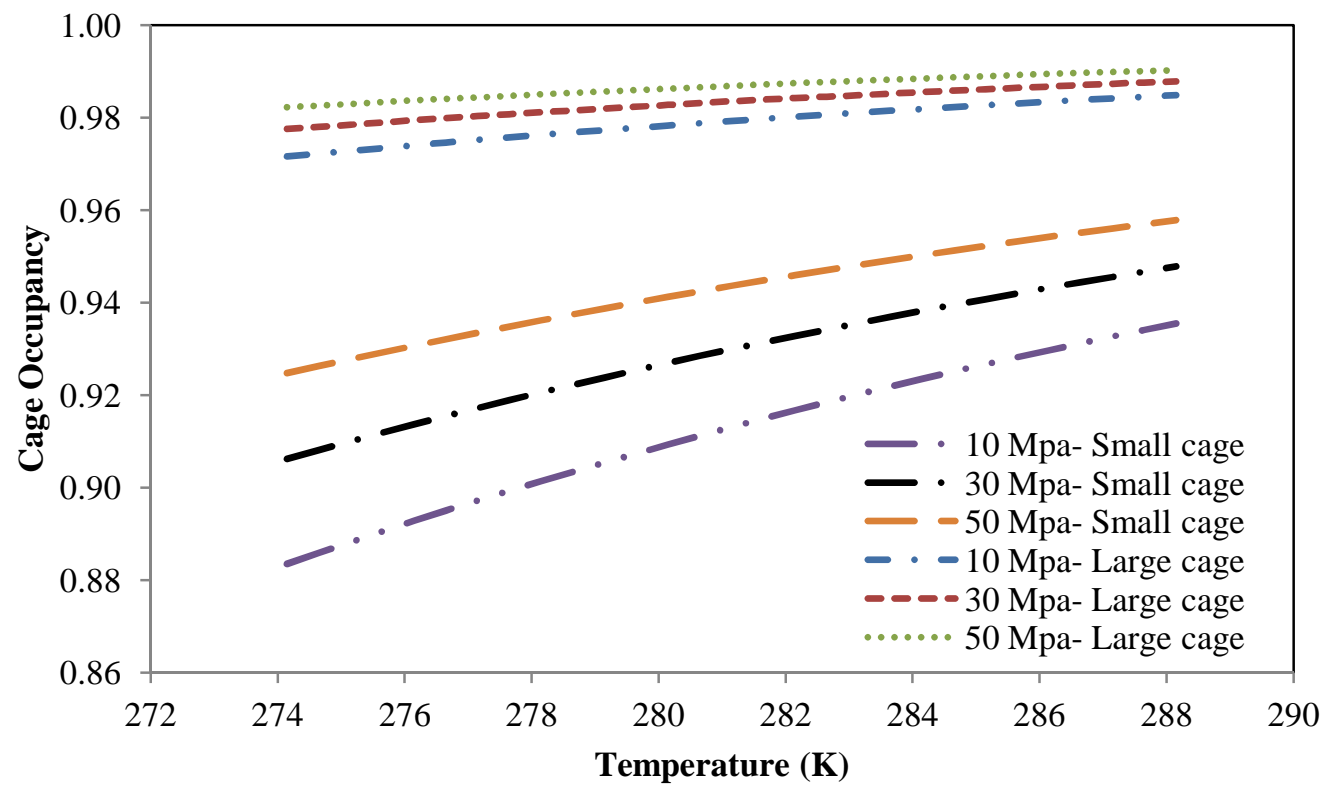

Figure 4.3 Cage occupancy of sI methane hydrate at $\mathrm{H}-\mathrm{L}_{\mathrm{w}}$ equilibrium predicted by this model

The solubility of ethane in water predicted by this model at $\mathrm{H}-\mathrm{L}_{\mathrm{w}}$ equilibrium is compared to the experimental data in Table 4.10. There is a limited experimental data available in the 
literature for ethane solubility in water when in equilibrium with gas hydrates ${ }^{69,72,155}$. The experimental data is of temperature at the range of $277-280 \mathrm{~K}$ and pressures at the range of 5-151 MPa. The average deviation (AD) values are shown in Table 4.10. For the data of Kim et al. ${ }^{69,72}$ the prediction shows the $\mathrm{AD}$ is less than $15 \%$ and $\mathrm{AAD}$ of $11.5 \%$. For the experimental data of Yang et al. ${ }^{155}$ the prediction shows high AD, this can be because of the unreliability of experimental data. ${ }^{77}$ As it can be observed from Table 4.10 that the temperature has no effect on the solubility of ethane in water at $\mathrm{H}-\mathrm{L}_{\mathrm{w}}$ equilibrium condition and cannot be considered reliable, even the data of Kim et al. ${ }^{69}$ show no temperature dependent and are also questionable about the reliability of the data. There is only one experimental data set available for the solubility of propane in water at $\mathrm{H}-\mathrm{L}_{\mathrm{w}}$ equilibrium condition. Table 4.11 shows the experimental data measured by Gaudatte and Servio ${ }^{74}$ and the prediction by this model. The AAD is $6.5 \%$ predicted by this model from experimental data. Considering the experimental uncertainties, this model is able to predict the pure hydrocarbon solubility in water in equilibrium with gas hydrate phase. 
Table 4.10 Solubility of ethane in water at $\mathrm{H}-\mathrm{L}_{\mathrm{w}}$ equilibrium

\begin{tabular}{|c|c|c|c|c|}
\hline $\begin{array}{c}\text { Temperature } \\
(\mathrm{K})\end{array}$ & $\begin{array}{l}\text { Pressure } \\
(\mathrm{MPa})\end{array}$ & $\begin{array}{c}\text { Experimental } \\
\text { mole fraction, } \\
x_{C_{2} H_{6}} \times 10^{4}\end{array}$ & $\begin{array}{l}\text { This model } \\
x_{C_{2} H_{6}}, \times 10^{4}\end{array}$ & $\% \mathrm{AD}$ \\
\hline \multicolumn{5}{|c|}{ Experimental data from Yang et al. $^{70}$} \\
\hline 273.10 & 51 & 4.12 & 3.40 & 17.48 \\
\hline 277.82 & 101 & 4.12 & 2.56 & 37.86 \\
\hline 278.46 & 151 & 4.12 & 1.94 & 52.91 \\
\hline \multicolumn{5}{|c|}{ Experimental data from Kim et al. ${ }^{69}$} \\
\hline 277.3 & 10.1 & 4.37 & 3.79 & 13.27 \\
\hline 277.8 & 15.1 & 4.37 & 3.84 & 12.13 \\
\hline 278.5 & 20.1 & 4.37 & 3.96 & 9.38 \\
\hline \multicolumn{5}{|c|}{ Experimental data from Kim et al. ${ }^{72}$} \\
\hline 277.7 & 10.1 & 3.53 & 3.93 & 11.33 \\
\hline 278.9 & 10.1 & 4.00 & 4.38 & 9.50 \\
\hline 280.0 & 10.1 & 4.50 & 4.84 & 7.56 \\
\hline 278.4 & 15.1 & 3.54 & 4.05 & 14.41 \\
\hline 279.5 & 15.1 & 3.97 & 4.48 & 12.85 \\
\hline 280.7 & 15.1 & 4.52 & 4.99 & 10.40 \\
\hline
\end{tabular}

Table 4.11 Solubility of Propane in water at $\mathrm{H}-\mathrm{L}_{\mathrm{w}}$ equilibrium

\begin{tabular}{ccccc}
\hline $\begin{array}{c}\text { Temperature } \\
(\mathrm{K})\end{array}$ & $\begin{array}{c}\text { Pressure } \\
(\mathrm{MPa})\end{array}$ & $\begin{array}{c}\text { Experimental }^{74} \\
\text { Mole fraction, } \\
x_{C_{3} \mathrm{H}_{8}, \times 10^{4}}\end{array}$ & $\begin{array}{c}\text { This model } \\
\text { mole fraction, } \\
x_{C_{3} \mathrm{H}_{8}, \times 10^{4}}\end{array}$ & \%AD \\
\hline 274.16 & 0.301 & 1.440 & 1.443 & 0.21 \\
274.23 & 0.253 & 1.439 & 1.458 & 1.32 \\
274.33 & 0.358 & 1.546 & 1.483 & 4.08 \\
275.20 & 0.302 & 1.572 & 1.704 & 8.40 \\
275.20 & 0.352 & 1.572 & 1.704 & 8.40 \\
276.16 & 0.355 & 1.642 & 1.890 & 15.10 \\
\hline
\end{tabular}




\subsubsection{H- $\mathrm{L}_{\mathrm{w}}$ Equilibrium for Mixed Components}

The model is extended to calculate the solubility of methane-ethane-propane mixture in water in equilibrium with mixed gas hydrates is at different temperatures and pressures. The composition of the hydrocarbon mixture consists of methane $\left(\mathrm{C}_{1}\right)$ 87.5\%, ethane $\left(\mathrm{C}_{2}\right) 8.1 \%$ and propane $\left(\mathrm{C}_{3}\right) 4.4 \%$. This composition is similar to the hydrocarbon composition of Macondo well in the Gulf of Mexico defined for endmembers methane, ethane and propane ${ }^{39-40}$. Figure 4.4 shows the prediction of methane-ethane-propane mixture solubility in water at $\mathrm{H}-\mathrm{L}_{\mathrm{w}}$ equilibrium at temperature range of $274-293 \mathrm{~K}$ and pressures up to $50 \mathrm{MPa}$. These temperature and pressure conditions are typically found in the marine environments. There is no experimental data available in the literature to our knowledge for the hydrocarbon mixture in two phase $\mathrm{H}-\mathrm{L}_{\mathrm{w}}$ equilibrium conditions. The $\mathrm{VL}_{\mathrm{w}} \mathrm{H}$ equilibrium is also show in the Figure 4.4 obtained for the same composition in gas phase. Above the $\mathrm{VL}_{\mathrm{w}} \mathrm{H}$ equilibrium is a two phase hydrate and liquid water $\left(\mathrm{H}-\mathrm{L}_{\mathrm{w}}\right)$ equilibrium and below the $\mathrm{VL}_{\mathrm{w}} \mathrm{H}$ is a complete dissociation of hydrate and a $\mathrm{V}-\mathrm{L}_{\mathrm{w}}$ equilibrium exits. The mixed hydrates forms at lower pressure than the pure methane hydrate. The presence of small amount of propane reduces the equilibrium pressure, as the pure propane forms at lower pressures. 


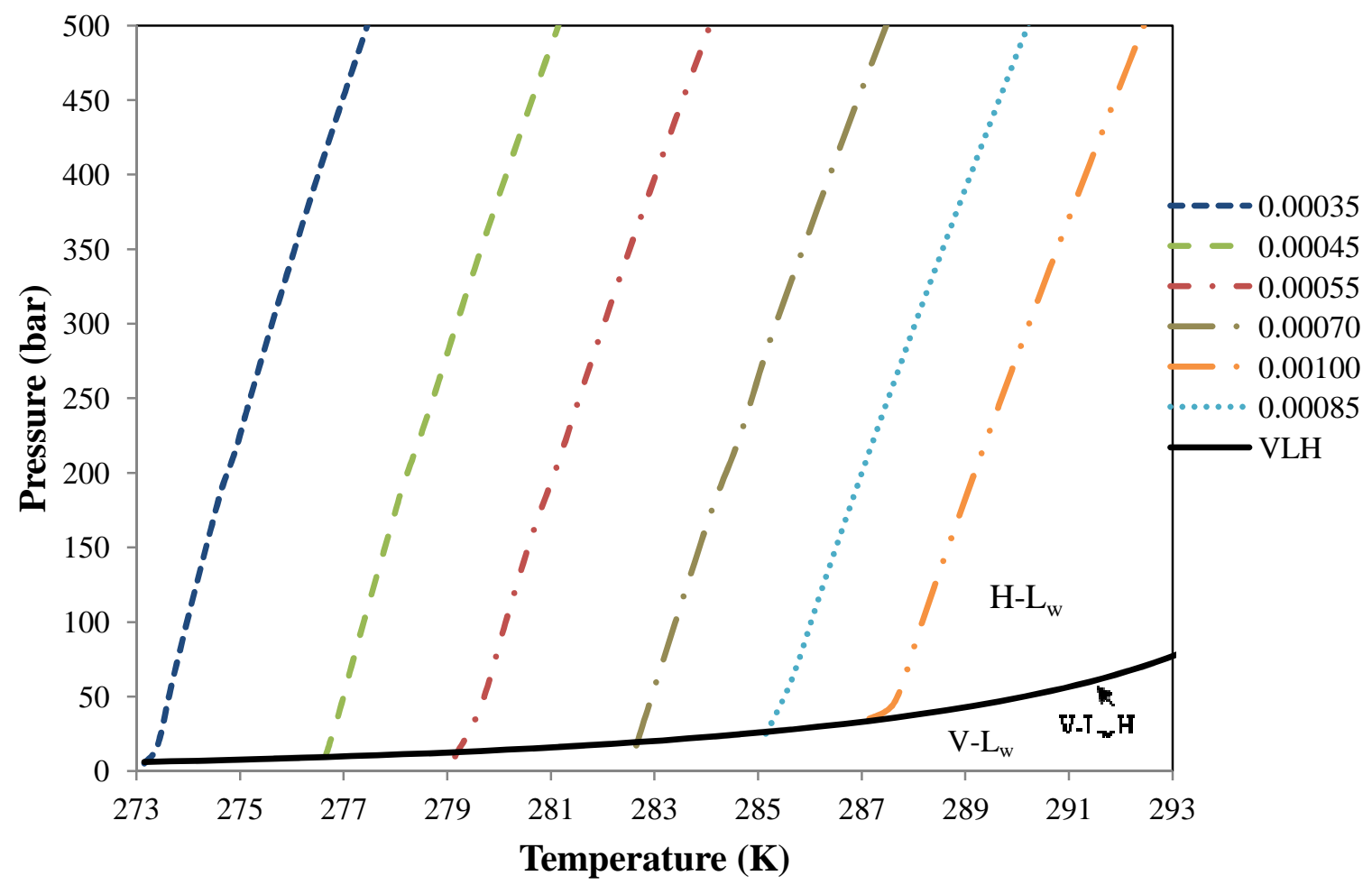

Figure 4.4 Dissolved hydrocarbon mixture concentration in the aqueous phase in equilibrium with gas hydrates at different temperatures and pressures. The vertical dotted lines are for total hydrocarbon mole fraction ( $\quad$ ) in aqueous phase with composition $\mathrm{C}_{1}=87.5 \%, \mathrm{C}_{2}=8.1 \%$ and $\mathrm{C}_{3}=4.4 \%$. Also shown is the $\mathrm{VL}_{\mathrm{w}} \mathrm{H}$ equilibrium curve with solid black line.

Structure II hydrate consists of 16 small cages and 8 large cages. The small cages are occupied with methane and the large cages with methane, ethane and propane. The fraction of occupied small and large cages of mixed hydrate plotted against temperature is shown in Figure 4.5. The cage occupancy for small cage increases with increase in temperature even though the concentration of hydrocarbon in the coexisting liquid is decreased. At lower hydrocarbon solubility in water, high pressures are required to have the $\mathrm{H}-\mathrm{L}_{\mathrm{w}}$ equilibrium and because of these higher pressures more amount of hydrocarbon transfer in to the hydrate phase increasing the cage occupancy. The increase in large cage occupancy is lesser than for the small cage occupancy primarily because the large cages are already mostly occupied. Most of the large cages are occupied 
by propane with some fraction of it occupied with ethane and even lesser by methane. Figure 4.6 shows the mole fraction of the hydrocarbon in the hydrate phase. The mole fraction of methane in the hydrate phase increases with the increase in temperature and with decrease in the solubility of hydrocarbon in water.
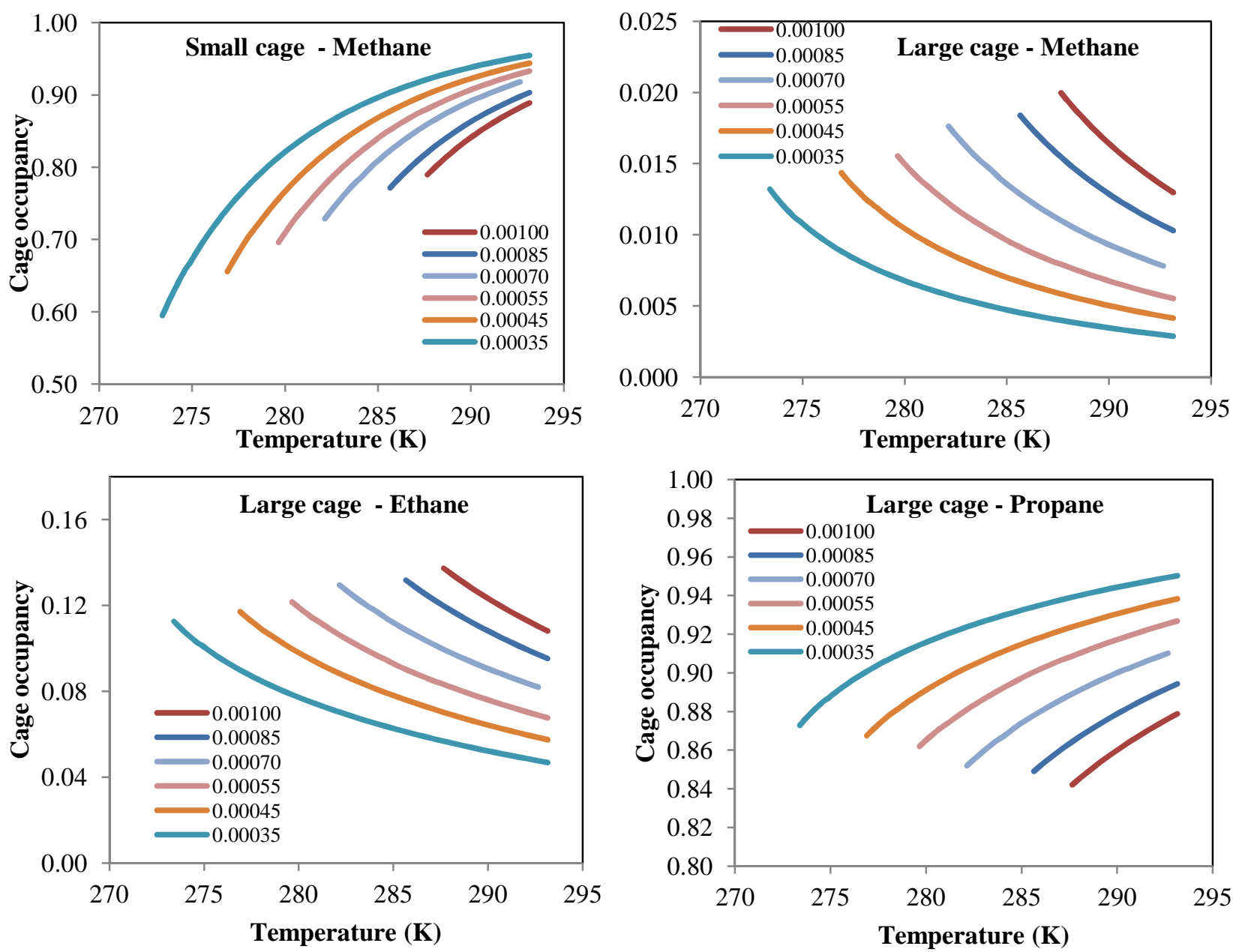

Figure 4.5 Gas hydrates cage occupancies at two phase equilibrium $\left(\mathrm{L}_{\mathrm{w}}-\mathrm{H}\right)$. The symbols are for different total hydrocarbon solubility in water with composition, $\mathrm{C}_{1}=87.5 \%, \mathrm{C}_{2}=8.1 \%$ and $\mathrm{C}_{3}=$ $4.4 \%$ when in equilibrium with hydrate phase 

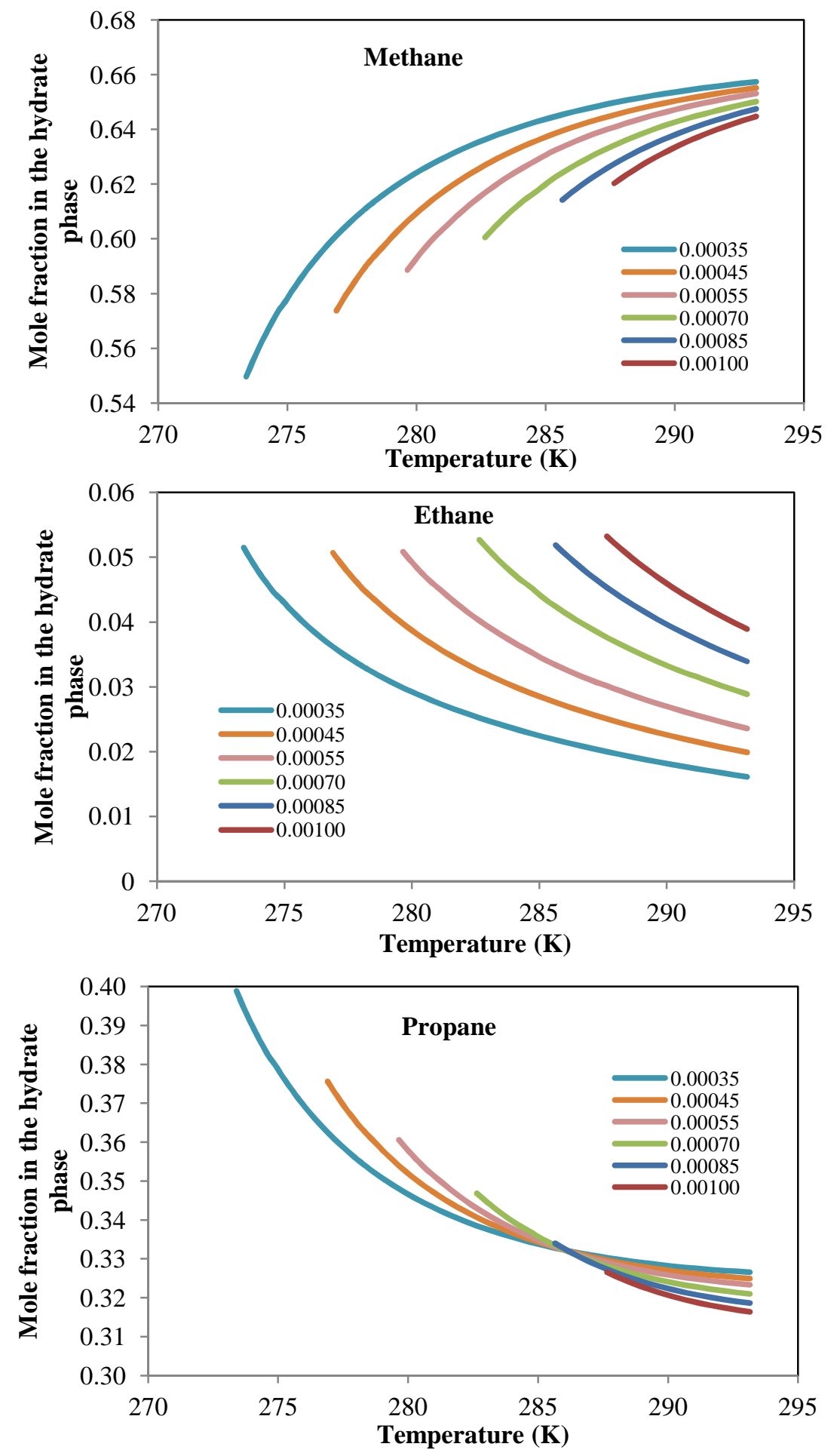

Figure 4.6 Hydrate phase composition at two phase equilibrium $\left(\mathrm{L}_{\mathrm{w}}-\mathrm{H}\right)$. The symbols are for different total hydrocarbon solubility in water with composition, $\mathrm{C}_{1}=87.5 \%, \mathrm{C}_{2}=8.1 \%$ and $\mathrm{C}_{3}=$ $4.4 \%$ when in equilibrium with hydrate phase. 


\subsubsection{H-Lw Equilibrium in Sea Water}

The concentration and type of the salts influence the solubility of hydrocarbons in water. The seawater has $35 \mathrm{wt} \%$ ions, the molality of the major ions taken from Millero ${ }^{156}$ are given in Table 4.12.

Table 4.12 Molality $\left(\mathrm{mol} / \mathrm{kg} \mathrm{H}_{2} \mathrm{O}\right)$ of ions in $35 \mathrm{wt} \%$ sea water concentration

\begin{tabular}{lc}
\hline Ions & Molality \\
\hline $\mathrm{Na}^{+}$ & 0.4850 \\
$\mathrm{Mg}^{2+}$ & 0.0552 \\
$\mathrm{Ca}^{2+}$ & 0.0106 \\
$\mathrm{~K}^{+}$ & 0.0106 \\
$\mathrm{Cl}^{-}$ & 0.5658 \\
$\mathrm{SO}_{4}{ }^{2-}$ & 0.0293 \\
$\mathrm{HCO}_{3}^{-}$ & 0.0024 \\
\hline
\end{tabular}

Using the Pitzer model for the calculation of activity of water and the Setschenow equation for the activity coefficents of the hydrocarbons in seawater, the $\mathrm{L}_{\mathrm{w}} \mathrm{H}$ equilibrium was calculated for methane in 35\% wt seawater and is given in Figure 4.7. In the calcualtion of propane activity coefficent, the $35 \mathrm{wt} \%$ seawater concentration was normalized for $\mathrm{NaCl}$ and $\mathrm{KCl}$ ions. Each vertical line in Figure 4.7 represents $P-T$ condition at two phase $\mathrm{H}-\mathrm{L}_{\mathrm{w}}$ equilibrium for a aqueous $\mathrm{CH}_{4}$ concentration. Also shown in the Figure 4.7 is the VLH equilibrium for seawater calcualted using our model. The aqueous solubilities of methane when in equilibrium with hydrate phase is calcualted and compared for fresh water and seawater to see the effect of salinity on the formation of hydrates and are given in Figure 4.8. 


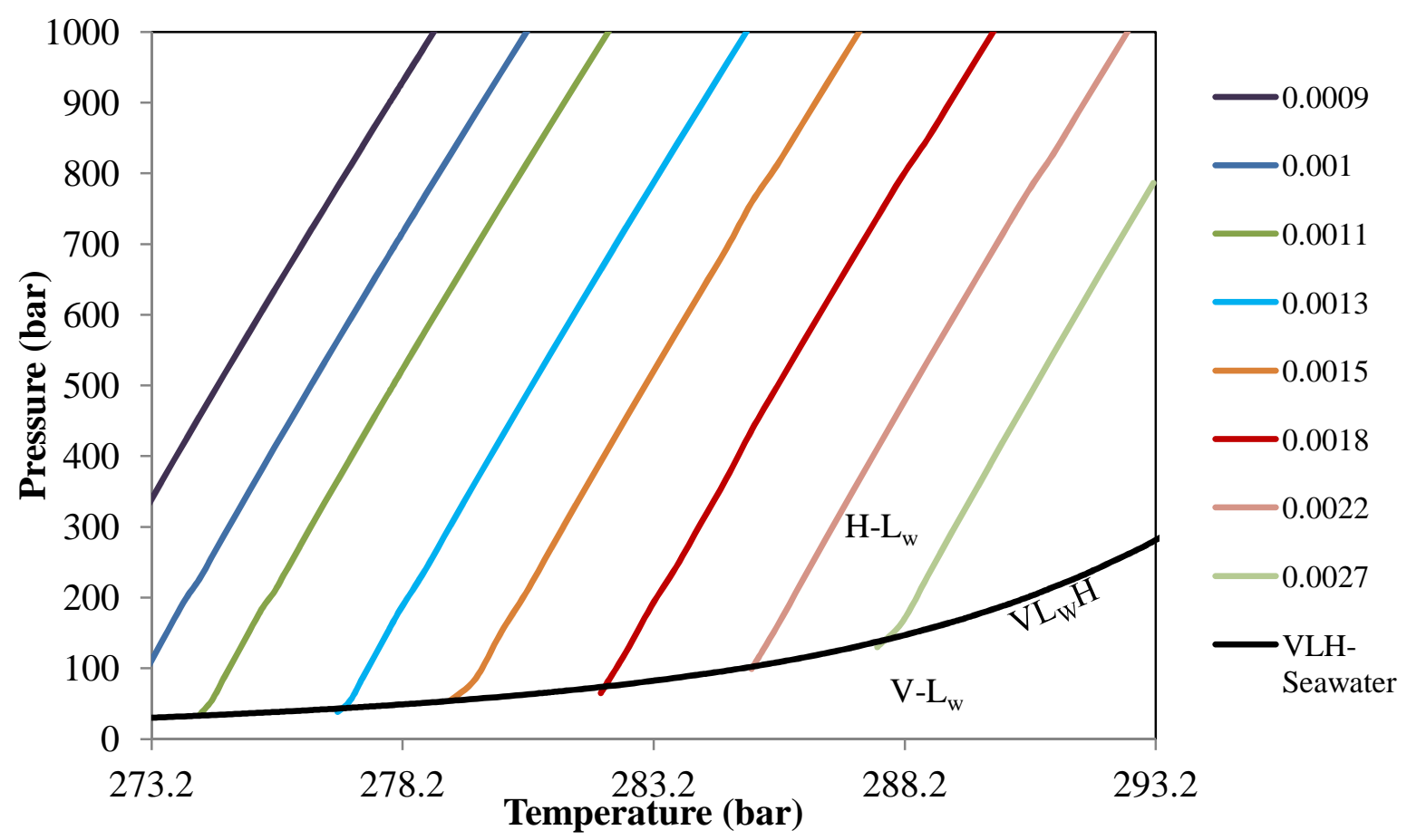

Figure 4.7 Solubility of methane in seawater at two phase hydrate -liquid seawater equilibrium. The vertical lines are aqueous methane mole fraction

As can be seen from Figure 4.8 that at a given temperature and aqueous hydrocarbon solubility, the hydrate forms at lower pressure in seawater than that in fresh water. This means that in two phase hydrate-liquid water equilibrium system, salts in seawater propmotes the hydrate formation instead of acting as inhibitors. It is well known that in the three phase VLH equilibrium, salts acts as an inhibitor to hydrate formation requiring higher pressure to form hydrate than to that of pure water at a given temperature. Figure 4.8 also shows the VLH equilibrium for seawater and pure water. The VLH for seawater is above to the VLH of pure water. When salts are present in the water, it lowers the chemical potential of aqueous water making the liquid phase more stable to that of hydrate phase and thus inhibiting the hydrate formation (requires higher pressures at the same temperature). Similar behavior was observed by Zhang et al. ${ }^{67}$ in their two phase $\mathrm{CO}_{2}$ hydrateliquid water system. 


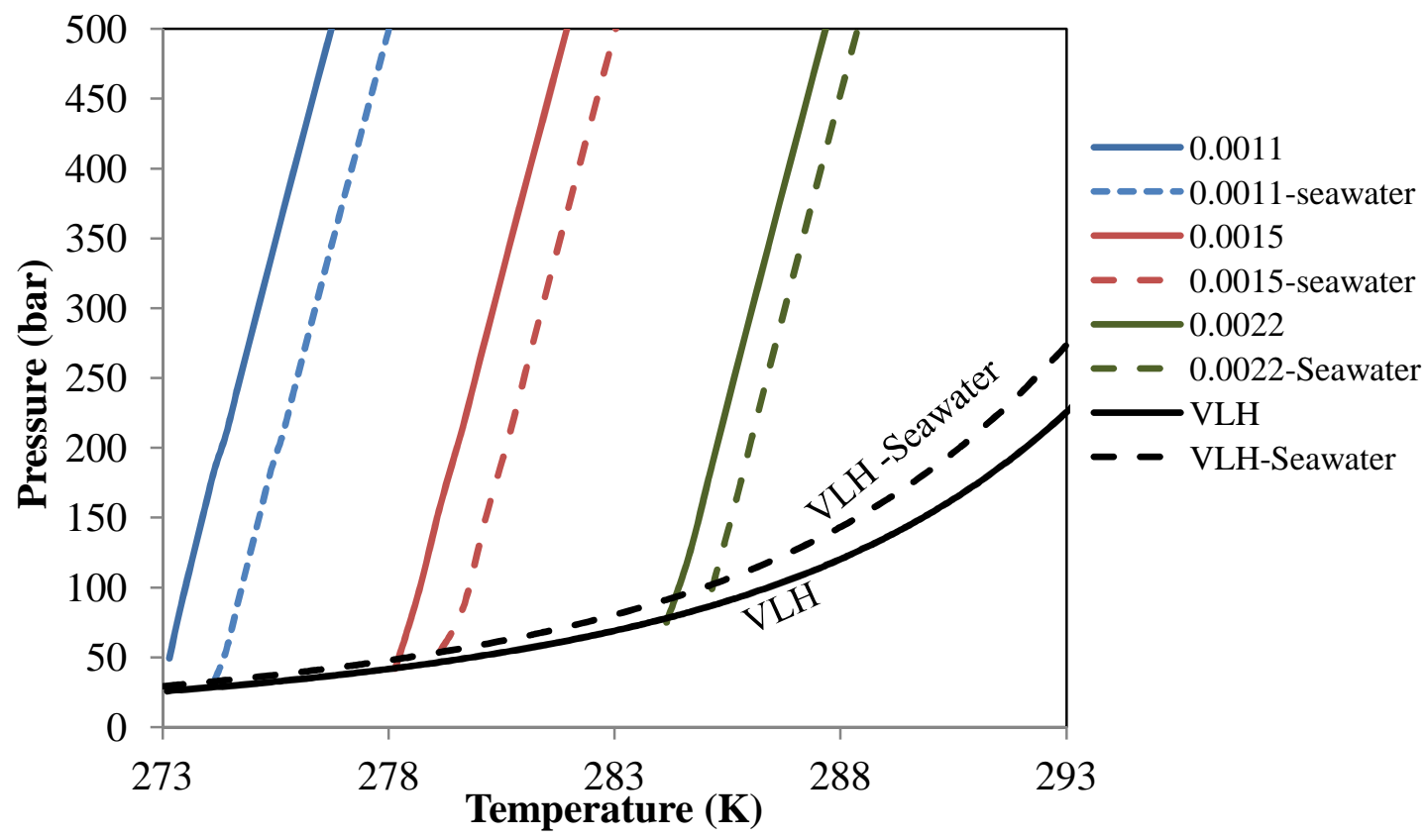

Figure 4.8 Effect of salinity in the formation of methane hydrate from the dissolved methane concentrations. The dotted lines are for seawater and solid lines are for pure water. The $\mathrm{VL}_{\mathrm{w}} \mathrm{H}$ equilibrium curve with solid black line and dotted line is for pure water and seawater respectively.

In the two phase V-L equilibrium, the chemical potential of the gas in the vapor and the gas in the liquid are equal. When salts are present in the liquid water, the chemical potential of the hydrocarbon in liquid will not change as it has to be equal to that of hydrocarbon in vapor chemical potential instead it will change the concentration of the gas in the liquid phase to match the chemical potentials. So for the V-L systems the concentration of the gas in the liquid will decrease when salts are present. At a temperature and pressure the increase in salinity will decrease the aqueous phase hydrocarbon solubility. In the $\mathrm{L}_{w} \mathrm{H}$ two phase system there is no vapor phase and the hydrocarbon concentration in the liquid phase is fixed $\left(\mathrm{CH}_{4}\right.$ aqueous solubility in this case). Thus, when electrolytes are added to the aqueous phase the chemical potential of the $\mathrm{CH}_{4}$ increases as the $\mathrm{CH}_{4}$ concentration is constant (no buffer unlike in the VL phase). However, the chemical potential of water in liquid is reduced because of the electrolytes. This reduction in the liquid water chemical 
potential is more than compensated by the increase in the chemical potential of $\mathrm{CH}_{4}$ which promotes the hydrate formation ${ }^{67}$. Thus, hydrates are formed at lower solubility in the presence of electrolyte to that of pure water at a given temperature and pressure. In other words, at a given aqueous hydrocarbon solubility hydrates forms at lower pressures in seawater than in pure water.

Figure 4.9 shows the solubility of mixed hydrocarbons in seawater and in fresh water. As we can see from the Figure that similar to the behavior of pure methane, the mixed hydrate phase equilibrium is also stable at lower pressures in seawater compare to that of in fresh water.

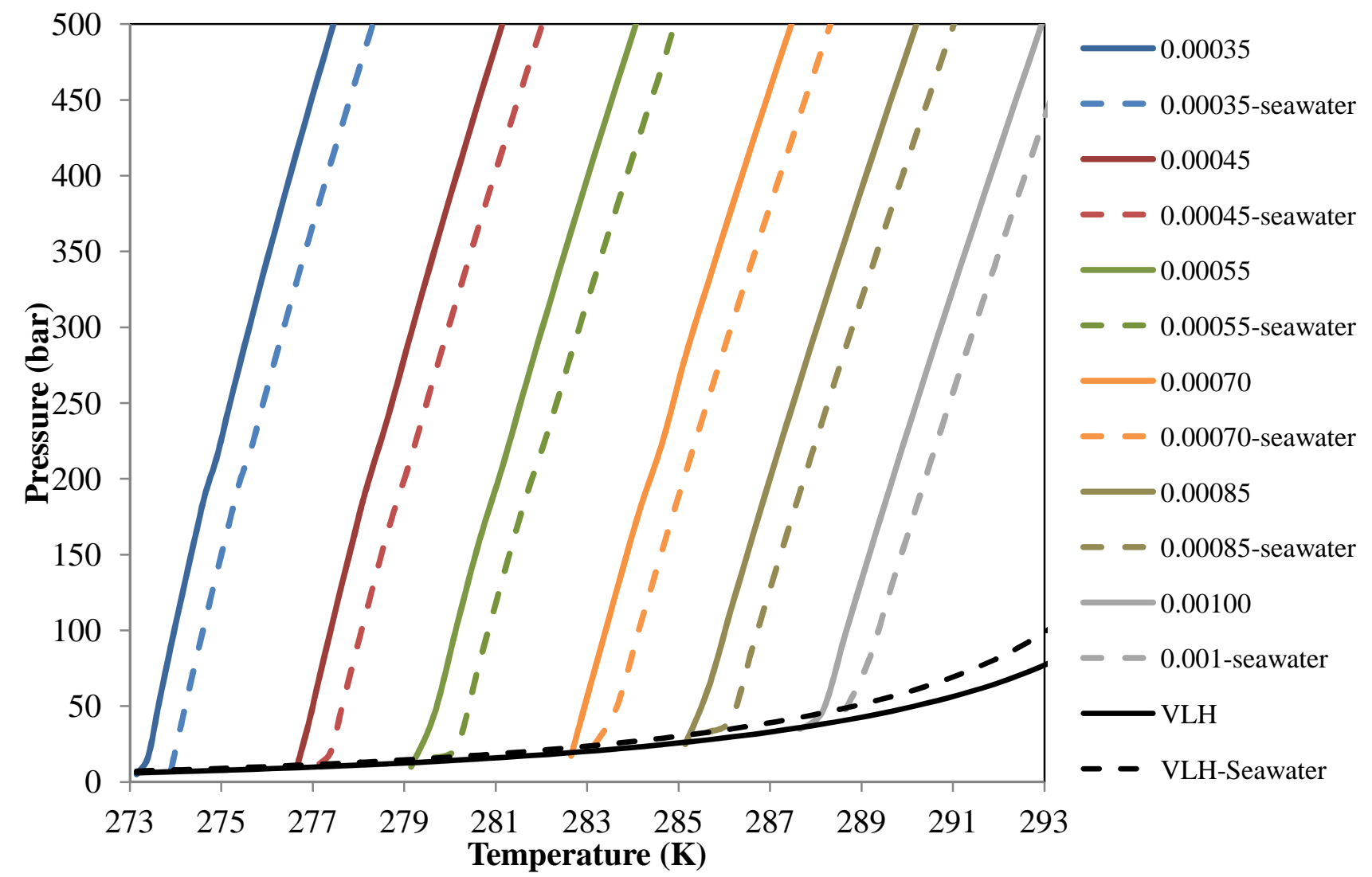

Figure 4.9 Dissolved hydrocarbon mixture concentration in the aqueous phase in equilibrium with gas hydrates at different temperatures and pressures for seawater and fresh water. The vertical dotted lines for seawater and solid lines for fresh water. Each vertical line is for total hydrocarbon mole fraction $\left(\boldsymbol{x}_{\boldsymbol{C}_{1} \boldsymbol{C}_{2} \boldsymbol{C}_{3}}\right)$ in aqueous phase with composition $\mathrm{C}_{1}=87.5 \%, \mathrm{C}_{2}=8.1 \%$ and $\mathrm{C}_{3}=4.4 \%$. Also shown is the $\mathrm{VL}_{\mathrm{w}} \mathrm{H}$ equilibrium curve for fresh water and seawater with solid black and dotted black line respectively. 


\subsection{Conclusions}

The two phase gas hydrate equilibrium is calculated based on the van der Waals and Platteeuw model and Holder model on the equality of the chemical potential difference concept. Variable reference chemical potential difference and enthalpy difference were used for each guest molecule and the Langmuir constants were calculated from the cell potential parameters. The model predictions were compared with the pure hydrocarbon solubility at $\mathrm{L}_{\mathrm{w}} \mathrm{H}$ with the available experimental data. The model is able to predict the experimental data well considering the experimental uncertainty without using any fitting parameters. Also, the cage occupancy of methane hydrate is predicted and compared to the experimental data. The cage occupancy increases with increase in temperature and pressure, and the large cage to small cage ratio decreases with increase in temperature and pressure. The solubility of methane-ethane-propane mixture in water at $\mathrm{H}-\mathrm{L}_{\mathrm{w}}$ equilibrium was predicted with this model at temperatures ranging from $274 \mathrm{~K}$ to $292 \mathrm{~K}$ and pressures up to $50 \mathrm{MPa}$. The model is also extended to calculate the aqueous hydrocarbon solubility at $\mathrm{H}-\mathrm{L}_{\mathrm{w}}$ for seawater. In the two phase $\mathrm{L}_{\mathrm{w}} \mathrm{H}$ region, the pressure required forming hydrate at a given solubility and temperature is less in seawater than in pure water. The presence of salts at mixed hydrocarbon promotes the hydrate formation instead of acting as the inhibitor for hydrate. This is in reverse to that in the three phase vapor-liquidwater-hydrate $\left(\mathrm{VL}_{\mathrm{w}} \mathrm{H}\right)$ region where it is well known that salts act as an inhibitor to hydrate formation. 


\subsection{Overall Conclusions and Recommendations}

\subsection{Conclusions}

The overall goal of this work is to better understand the stability of the methane-ethanepropane mixed structure II (sII) hydrate at pressures which are closer to the deep water conditions. Molecular dynamics (MD) simulations were carried out to study the dissolution of sII mixed hydrate by varying the cage occupancies of ethane and propane in the large cages and also methane both in large and small cages. The pure and mixture hydrocarbon solubilities in the water at two phase $H-L_{w}$ equilibrium were calculated based on the van der Waals and Platteeuw solid theory and Holder model for hydrocarbon fugacity and chemical potential of water in liquid phase.

A structure II simulation box was build with methane-ethane-propane as the guest molecules. The intermolecular potentials used in the simulations were verified by calculating the lattice constant of sII hydrate. The lattice constant increases with the increase in temperature. The lattice constant obtained at $275 \mathrm{~K}$ is $17.32 \AA$ compare to the experimental value of $17.38 \AA$.

MD simulations were performed on the 48 unit cell of sII hydrate by varying the cage occupancy. In all the simulations performed there was no dissolution of ethane or propane molecules was observed only the methane molecules moved from the hydrate phase to the liquid water phase. MD simulations reveals that the fully occupied hydrate was found to be more stable as less number of methane molecules moved to the liquid water phase and this number increased with the decrease in the large cage occupancy.

$>$ There was no increase in the number of methane molecules with the decrease in the small cage occupancy from 100 to $81.25 \%$. The small cages are more stable than the large cages and these empty small cages $(81.25 \%)$ do not destabilize the sII hydrate crystal. The 
dissolution of sII mixed hydrate is specific to the cage specific occupancy, not just depends on the overall occupancy.

From the visual view of the simulation using VMD, the methane molecules moved from the hydrate to liquid water phase mostly came from the large cages. There were lot of small cages breakup and formation was observed at the hydrate-liquid water interface.

The dissolution of hydrate was linear in the initial few nanoseconds of the simulation, after this there was lot of oscillatory behavior was observed. The oscillatory behavior is due to the hydrate formation and dissociation of the cages at the interface.

$>$ There was no particular trend observed in the dissolution of sII hydrate by varying methane, ethane and propane cage occupancy in the large cage.

$>$ The amount of dissolution of sI methane hydrate is more than the sII mixed hydrate when the cage occupancy was decreased from $100 \%$ to $50 \%$.

The aqueous hydrocarbon solubilities at two phase hydrate-liquid water equilibrium was predicted for pure hydrocarbons and for the mixture in pure water and in seawater.

The Langmuir constants were calculated from the cell potential parameters which were obtained from ab initio intermolecular potentials. Variable reference parameters were used for each guest and for the mixture the reference parameters were calculated by using mixing rules from the pure reference parameters.

The aqueous hydrocarbon solubilites obtained from the thermodynamic model were compared to the available experimental data for methane, ethane and propane. The model is able to predict the experimental data well considering the experimental accuracy. 
The model is extended to predict the mixed hydrocarbon solubilties at two phase $\mathrm{H}-\mathrm{L}_{\mathrm{w}}$ equilibrium at deepsea conditions for the composition of Macondo oil spill in the Gulf of Mexico as a typical thermogenic hydrocarbon mixture.

The pressure required for the formation of hydrate is reduced with the increase in the salinity in the liquid water at a given temperature and hydrocarbon solubility. Thus, the electrolytes act as hydrate promoters rather than acting as a inhibitors in a two phase $\mathrm{H}-\mathrm{L}_{\mathrm{w}}$ equilibrium.

\subsection{Recommendations}

$>$ The size of the water molecules box is not big enough to dissolve the propane molecules. Molecular dynamics simulations have to be performed with larger number of the liquid water molecules in the liquid water box which can dissolve more number of propane and ethane molecules.

Investigation has to be done to see if further decrease, lower than $81.25 \%$, in small cage occupancy have any influence in the dissolution of the mixed sII hydrate.

$>$ Investigation on the effect of water phase undersaturation with respect to the gas forming hydrate on the dissolution rates. The levels of undersaturation will reduce the driving force of the dissolution.

Fugacity of hydrocarbon is calculated using the Henry's law method with the pyonting correction. The Henry's constant was obtained by fitting to the experimental V-L equilibrium data. A new method has to be used to calculate the fugacity of hydrocarbon in the liquid phase. 
$>$ The experimental data on $\mathrm{H}-\mathrm{L}_{\mathrm{w}}$ equilibrium is very limited and there is no experimental data covering the mixed gas hydrates which form sII. Accurate amount of experimental data is required for pure hydrocarbons and for the mixture at two phase $\mathrm{H}-\mathrm{L}_{\mathrm{w}}$ equilibrium. 


\subsection{References}

1. Powel, H. J. M., J. Chem. Soc. 1948, 61.

2. Davy, H. P., Trans. Soc. London 1811, 101, 1.

3. Priestley, J., Experiments and observations on different kinds of air, and other branches of natural philosophy, connected with the subject. Printed by Thomas Pearson, and sold by J. Johnson: 1790; Vol. 3.

4. Wroblewski, S. c., On the laws of solubility of the carbonic acid in water at high pressures. Acad. Sci. Paris 1882, 1355-1357.

5. Wroblewski, S. O. 1. F., On the composition of the hydrate of the carbonic acid. . Acad. Sci. Paris 1882, 954-958.

6. Hammerschmidt, E., Formation of gas hydrates in natural gas transmission lines. Ind. Eng. Chem. 1934, 26, (8), 851-855.

7. Makogon, Y. F., Gas hydrates: frozen energy. Recherche 1987, 18, (192), 1192.

8. Trofimuk, A.; Makogon, Y. F.; Tolkachev, M., Gas hydrate accumulations-new reserve of energy sources. Geologiya nefti i gaza 1981, 10, 15-22.

9. Solov'ev, V., Global estimation of gas content in submarine gas hydrate accumulations. Geol Geofiz 2002, 43, (7), 648-661.

10. Klauda, J.; Sandler, S., Global distribution of methane hydrate in ocean sediment. Energy Fuels 2005, 19, (2), 459-470.

11. Holder, G.; John, V.; Yen, S. In Geological implications of gas production from In-situ gas hydrates, 1980; 1980.

12. Kvenvolden, K. A., Methane hydrate--A major reservoir of carbon in the shallow geosphere? Chem. Geol. 1988, 71, (1-3), 41-51. 
13. Park, Y.; Kim, D.; Lee, J.; Huh, D.; Park, K.; Lee, J.; Lee, H., Sequestering carbon dioxide into complex structures of naturally occurring gas hydrates. Proceedings of the National Academy of Sciences 2006, 103, (34), 12690.

14. Barduhn, A. J.; Towlson, H. E.; Hu, Y. C., The properties of some new gas hydrates and their use in demineralizing sea water. AlChE J. 1962, 8, (2), 176-183.

15. Kang, S. P.; Lee, H., Recovery of $\mathrm{CO} 2$ from flue gas using gas hydrate: thermodynamic verification through phase equilibrium measurements. Environmental science \& technology 2000, 34, (20), 4397-4400.

16. Miller, S.; Smythe, W., Carbon dioxide clathrate in the martian ice cap. Science 1970, 170, (3957), 531.

17. Davidson, D.; El-Defrawy, M.; Fuglem, M.; Judge, A. In Natural gas hydrates in northern Canada, 2010; 2010; pp 937-943.

18. Zhang, Y.; Xu, Z., Kinetics of convective crystal dissolution and melting, with applications to methane hydrate dissolution and dissociation in seawater. Earth. Planet. Sci. Lett. 2003, 213, (1), 133-148.

19. Sloan, E.; Koh, C., Clathrate hydrates of natural gases. CRC Press: 2007.

20. Sassen, R.; MacDonald, I. R., Evidence of structure H hydrate, Gulf of Mexico continental slope. Org. Geochem. 1994, 22, (6), 1029-1032.

21. McMullan, R. K.; Jeffrey, G., Polyhedral clathrate hydrates. IX. Structure of ethylene oxide hydrate. J. Chem. Phys. 1965, 42, 2725.

22. Ripmeester, J.; Ratcliffe, C., The Diverse Nature of Dodecahedral Cages in Clathrate Hydrates As Revealed by ${ }^{129} \mathrm{Xe}$ and ${ }^{13} \mathrm{C}$ NMR Spectroscopy: $\mathrm{CO}_{2}$ as a Small-Cage Guest. Energy Fuels 1998, 12, (2), 197-200. 
23. Kvenvolden, K. A., A review of the geochemistry of methane in natural gas hydrate. Org. Geochem. 1995, 23, (11-12), 997-1008.

24. Milkov, A. V.; Claypool, G. E.; Lee, Y. J.; Sassen, R., Gas hydrate systems at Hydrate Ridge offshore Oregon inferred from molecular and isotopic properties of hydrate-bound and void gases. Geochim. Cosmochim. Acta 2005, 69, (4), 1007-1026.

25. Torres, M.; Wallmann, K.; Trehu, A.; Bohrmann, G.; Borowski, W.; Tomaru, H., Gas hydrate growth, methane transport, and chloride enrichment at the southern summit of Hydrate Ridge, Cascadia margin off Oregon. Earth. Planet. Sci. Lett. 2004, 226, (1-2), 225241.

26. Haeckel, M.; Suess, E.; Wallmann, K.; Rickert, D., Rising methane gas bubbles form massive hydrate layers at the seafloor. Geochim. Cosmochim. Acta 2004, 68, (21), 43354345.

27. Brooks, J.; Kennicutt, M.; Fay, R.; McDonald, T.; Sassen, R., Thermogenic gas hydrates in the Gulf of Mexico. Science 1984, 225, (4660), 409.

28. Pohlman, J. W.; Canuel, E. A.; Chapman, N. R.; Spence, G. D.; Whiticar, M. J.; Coffin, R. B., The origin of thermogenic gas hydrates on the northern Cascadia Margin as inferred from isotopic $(13 \mathrm{C} / 12 \mathrm{C}$ and $\mathrm{D} / \mathrm{H})$ and molecular composition of hydrate and vent gas. Org. Geochem. 2005, 36, (5), 703-716.

29. Buffett, B.; Archer, D., Global inventory of methane clathrate: sensitivity to changes in the deep ocean. Earth. Planet. Sci. Lett. 2004, 227, (3-4), 185-199.

30. Rehder, G.; Kirby, S.; Durham, W.; Stern, L.; Peltzer, E.; Pinkston, J.; Brewer, P., Dissolution rates of pure methane hydrate and carbon-dioxide hydrate in undersaturated seawater at 1000-m depth. Geochim. Cosmochim. Acta 2004, 68, (2), 285-292. 
31. Chapman, R.; Pohlman, J.; Coffin, R.; Chanton, J.; Lapham, L., Thermogenic gas hydrates in the northern Cascadia Margin. Eos Trans. AGU 2004, 85, 361-365.

32. Davies, S.; Selim, M.; Sloan, E.; Bollavaram, P.; Peters, D., Hydrate plug dissociation. AlChE J. 2006, 52, (12), 4016-4027.

33. Rehder, G.; Leifer, I.; Brewer, P. G.; Friederich, G.; Peltzer, E. T., Controls on methane bubble dissolution inside and outside the hydrate stability field from open ocean field experiments and numerical modeling. Mar. Chem. 2009, 114, (1-2), 19-30.

34. Rehder, G.; Brewer, P. W.; Peltzer, E. T.; Friederich, G., Enhanced lifetime of methane bubble streams within the deep ocean. Geophysical research letters 2002, 29, (15), 21-1.

35. McNutt, M.; Camilli, R.; Guthrie, G. H. H.; Labson, V.; Lehr, B. M. D.; Ratzel, A.; Sogge, M., Assessment of Flow Rate Estimates for the Deepwater Horizon/Macondo Well Oil Spill Flow Rate Technical Group report to the National Incident Command, Interagency Solutions Group. Washington, DC: US Department of Interior. In 2011.

36. Hu, L.; Yvon-Lewis, S. A.; Kessler, J. D.; MacDonald, I. R., Methane fluxes to the atmosphere from deepwater hydrocarbon seeps in the northern Gulf of Mexico. Journal of Geophysical Research 2012, 117, (C1), C01009.

37. Warzinski, R. P.; Riestenberg, D. E.; Gabitto, J.; Haljasmaa, I. V.; Lynn, R. J.; Tsouris, C., Formation and behavior of composite $\mathrm{CO}<\mathrm{sub}>2</ \mathrm{sub}>$ hydrate particles in a highpressure water tunnel facility. Chem. Eng. Sci. 2008, 63, (12), 3235-3248.

38. Warzinski, R. P.; Lynn, R. J.; Robertson, A. M.; Haljasmaa, I. V., DEVELOPMENT OF A HIGH-PRESSURE WATER TUNNEL FACILITY FOR OCEAN CO2 STORAGE EXPERIMENTATION. Preprint Papers-American Chemical Society, Division of Fuel Chemistry 2000, 45, (4), 809-813. 
39. Valentine, D. L.; Kessler, J. D.; Redmond, M. C.; Mendes, S. D.; Heintz, M. B.; Farwell, C.; Hu, L.; Kinnaman, F. S.; Yvon-Lewis, S.; Du, M., Propane respiration jump-starts microbial response to a deep oil spill. Science 2010, 330, (6001), 208-211.

40. Reddy, C. M.; Arey, J. S.; Seewald, J. S.; Sylva, S. P.; Lemkau, K. L.; Nelson, R. K.; Carmichael, C. A.; McIntyre, C. P.; Fenwick, J.; Ventura, G. T., Composition and fate of gas and oil released to the water column during the Deepwater Horizon oil spill. Proceedings of the National Academy of Sciences 2012, 109, (50), 20229-20234.

41. Hester, K.; Peltzer, E.; Walz, P.; Dunk, R.; Sloan, E.; Brewer, P., A natural hydrate dissolution experiment on complex multi-component hydrates on the sea floor. Geochim. Cosmochim. Acta 2009, 73, (22), 6747-6756.

42. Hester, K.; Dunk, R.; White, S. N.; Brewer, P. G.; Peltzer, E. T.; Sloan, E., Gas hydrate measurements at Hydrate Ridge using Raman spectroscopy. Geochim. Cosmochim. Acta 2007, 71, (12), 2947-2959.

43. Lapham, L. L.; Chanton, J. P.; Chapman, R.; Martens, C. S., Methane under-saturated fluids in deep-sea sediments: Implications for gas hydrate stability and rates of dissolution. Earth. Planet. Sci. Lett. 2010.

44. Nihous, G.; Masutani, S., Notes on the dissolution rate of gas hydrates in undersaturated water. Chem. Eng. Sci. 2006, 61, (23), 7827-7830.

45. Bigalke, N.; Rehder, G.; Gust, G., Methane hydrate dissolution rates in undersaturated seawater under controlled hydrodynamic forcing. Mar. Chem. 2009, 115, (3-4), 226-234.

46. Gabitto, J.; Tsouris, C., Dissolution mechanisms of $\mathrm{CO} 2$ hydrate droplets in deep seawaters. Energy Convers. Manage. 2006, 47, (5), 494-508. 
47. Brewer, P. G.; Peltzer, E. T.; Friederich, G.; Rehder, G., Experimental determination of the fate of rising CO2 droplets in seawater. Environ. Sci. Technol 2002, 36, (24), 5441-5446.

48. Shindo, Y.; Hakuta, T.; Fujioka, Y.; Takeuchi, K.; Komiyama, H., Controlling effect of $\mathrm{CO} 2$ hydrate membrane on $\mathrm{CO} 2$ dissolution into water from the surface of liquid $\mathrm{CO} 2$. Energy Convers. Manage. 1995, 36, (6-9), 479-484.

49. Mori, Y. H.; Murakami, T., Can the thickness of hydrate films on CO2 drops in seawater be estimated from their buoyant motion?-A critique of a previous paper. Energy Convers. Manage. 2007, 48, (2), 494-499.

50. Egorov, A.; Crane, K.; Vogt, P.; Rozhkov, A.; Shirshov, P., Gas hydrates that outcrop on the sea floor: stability models. Geo-Marine Letters 1999, 19, (1), 68-75.

51. English, N.; Johnson, J.; Taylor, C., Molecular-dynamics simulations of methane hydrate dissociation. J. Chem. Phys. 2005, 123, 244503.

52. Jiang, H.; Myshakin, E. M.; Jordan, K. D.; Warzinski, R. P., Molecular Dynamics Simulations of the Thermal Conductivity of Methane Hydrate. J. Phys. Chem. B 2008, 112, (33), 10207-10216.

53. Baez, L. A.; Clancy, P., Computer Simulation of the Crystal Growth and Dissolution of Natural Gas Hydratesa. Ann. N.Y. Acad. Sci. 1994, 715, (1), 177-186.

54. Radhakrishnan, R.; Trout, B. L., A new approach for studying nucleation phenomena using molecular simulations: Application to CO hydrate clathrates. J. Chem. Phys. 2002, 117, 1786.

55. Westacott, R.; Rodger, P., Full-coordinate free-energy minimisation for complex molecular crystals: type I hydrates. Chem. Phys. Lett. 1996, 262, (1-2), 47-51. 
56. Myshakin, E. M.; Jiang, H.; Warzinski, R. P.; Jordan, K. D., Molecular Dynamics Simulations of Methane Hydrate Decomposition†. J. Phys. Chem. A 2009, 113, (10), 19131921.

57. Hirai, S.; Okazaki, K.; Kuraoka, S.; Kawamura, K., Study for the stability of CO2 clathratehydrate using molecular dynamics simulation. Energy Convers. Manage. 1996, 37, (6-8), 1087-1092.

58. Kumagai, N.; Kawamura, K.; Yokokawa, T., An interatomic potential model for H 2 O: applications to water and ice polymorphs. Molecular Simulation 1994, 12, (3), 177-186.

59. Kvamme, B.; Kuznetsova, T., Investigation into stability and interfacial properties of hydrate-aqueous fluid system. Mathematical and Computer Modelling 2010, 51, (3-4), 156159.

60. Sarupria, S.; Debenedetti, P. G., Molecular Dynamics Study of Carbon Dioxide Hydrate Dissociation. J. Phys. Chem. A 2011.

61. Potoff, J.; Siepmann, J., Vapor-liquid equilibria of mixtures containing alkanes, carbon dioxide, and nitrogen. AlChE J. 2001, 47, (7), 1676-1682.

62. Maini, B. B.; Bishnoi, P., Experimental investigation of hydrate formation behaviour of a natural gas bubble in a simulated deep sea environment. Chem. Eng. Sci. 1981, 36, (1), 183189.

63. Davie, M.; Zatsepina, O. Y.; Buffett, B., Methane solubility in marine hydrate environments. Marine geology 2004, 203, (1), 177-184.

64. McGinnis, D.; Greinert, J.; Artemov, Y.; Beaubien, S.; Wuest, A., Fate of rising methane bubbles in stratified waters: How much methane reaches the atmosphere? Journal of Geophysical Research-Oceans 2006, 111, C09007. 
65. Hashemi, S.; Macchi, A.; Bergeron, S.; Servio, P., Prediction of methane and carbon dioxide solubility in water in the presence of hydrate. Fluid Phase Equilib. 2006, 246, (1), 131-136.

66. Servio, P.; Englezos, P., Measurement of dissolved methane in water in equilibrium with its hydrate. J. Chem. Eng. Data 2002, 47, (1), 87-90.

67. Zhang, Y.; Holder, G. D.; Warzinski, R. P., Phase equilibrium in two-phase, water-richliquid, hydrate systems: experiment and theory. Ind. Eng. Chem. Res. 2008, 47, (2), 459469.

68. Lu, W.; Chou, I. M.; Burruss, R. C., Determination of methane concentrations in water in equilibrium with sI methane hydrate in the absence of a vapor phase by $\langle\mathrm{i}>$ in $\operatorname{situ}</ \mathrm{i}\rangle$ Raman spectroscopy. Geochim. Cosmochim. Acta 2008, 72, (2), 412-422.

69. Kim, Y.; Ryu, S.; Yang, S.; Lee, C., Liquid water-hydrate equilibrium measurements and unified predictions of hydrate-containing phase equilibria for methane, ethane, propane, and their mixtures. Ind. Eng. Chem. Res. 2003, 42, (11), 2409-2414.

70. Yang, S.; Cho, S.; Lee, H.; Lee, C., Measurement and prediction of phase equilibria for water+ methane in hydrate forming conditions. Fluid Phase Equilib. 2001, 185, (1), 53-63.

71. Seo, Y.; Lee, H.; Ryu, B. J., Hydration number and two-phase equilibria of CH4 hydrate in the deep ocean sediments. Geophysical Research Letters 2002, 29, (8), 85-1-85-4.

72. Kim, Y.; Lim, B.; Lee, J.; Lee, C., Solubilities of carbon dioxide, methane, and ethane in sodium chloride solution containing gas hydrate. J. Chem. Eng. Data 2008, 53, (6), 13511354. 
73. Lu, W.; Chou, I. M.; Burruss, R. C., Determination of methane concentrations in water in equilibrium with sI methane hydrate in the absence of a vapor phase by in situ Raman spectroscopy. Geochim. Cosmochim. Acta 2008, 72, (2), 412-422.

74. Gaudette, J.; Servio, P., Measurement of dissolved propane in water in the presence of gas hydrate. Journal of Chemical \& Engineering Data 2007, 52, (4), 1449-1451.

75. Holder, G. D.; Mokka, L. P.; Warzinski, R. P., Formation of gas hydrates from single-phase aqueous solutions. Chem. Eng. Sci. 2001, 56, (24), 6897-6903.

76. Sun, R.; Duan, Z., An accurate model to predict the thermodynamic stability of methane hydrate and methane solubility in marine environments. Chem. Geol. 2007, 244, (1-2), 248262.

77. Mohammadi, A. H.; Richon, D., Thermodynamic model for predicting liquid water-hydrate equilibrium of the water-hydrocarbon system. Ind. Eng. Chem. Res. 2008, 47, (4), 13461350.

78. Anderson, B.; Bazant, M.; Tester, J.; Trout, B., Application of the cell potential method to predict phase equilibria of multicomponent gas hydrate systems. Journal of Physical Chemistry B-Condensed Phase 2005, 109, (16), 8153-8163.

79. Parrish, W. R.; Prausnitz, J. M., Dissociation pressures of gas hydrates formed by gas mixtures. Ind. Eng. Chem. Proc. DD 1972, 11, (1), 26-35.

80. Sparks, K. A.; Jefferson, W.; Cao, Z.; Trout, B. L., Configurational properties of water clathrates: Monte Carlo and multidimensional integration versus the Lennard-Jones and Devonshire approximation. J. Phys. Chem. B 1999, 103, (30), 6300-6308. 
81. Cao, Z.; Anderson, B.; Tester, J.; Trout, B. In Development and Application of an Ab Initio Methane-Water Potential for the Study of Phase Equilibria of Methane Hydrates, 2002; 2002; pp 418-446.

82. John, V.; Holder, G., Contribution of second and subsequent water shells to the potential energy of guest-host interactions in clathrate hydrates. J. Phys. Chem. 1982, 86, (4), 455459.

83. Klauda, J.; Sandler, S., A fugacity model for gas hydrate phase equilibria. Ind. Eng. Chem. Res 2000, 39, (9), 3377-3386.

84. John, V.; Holder, G., Choice of cell size in the cell theory of hydrate phase gas-water interactions. J. Phys. Chem. 1981, 85, (13), 1811-1814.

85. Handa, Y. P., Effect of hydrostatic pressure and salinity on the stability of gas hydrates. $J$. Phys. Chem. 1990, 94, (6), 2652-2657.

86. Makogon, I. U. F., Hydrates of hydrocarbons. Pennwell Corp: 1997.

87. Allen, M. P., Introduction to molecular dynamics simulation. Computational Soft Matter: From Synthetic Polymers to Proteins 2004, 23, 1-28.

88. Tester, J. W.; Bivins, R. L.; Herrick, C., Use of Monte Carlo in calculating the thermodynamic properties of water clathrates. AlChE J. 1972, 18, (6), 1220-1230.

89. van der Spoel, D.; Lindahl, E.; Hess, B.; Van Buuren, A.; Apol, E.; Meulenhoff, P.; Tieleman, D.; Sijbers, A.; Feenstra, K.; van Drunen, R., Gromacs User Manual version 3.3. 2008.

90. Argunova, K. K.; Bondarev, E. A.; Rozhin, I. I., Environmental and engineering geology: study of the effect of oil producing wells of the Vankor field on temperature conditions of rocks. Inzhenernaya Ekologiya 2009, (2), 43-55. 
91. Barrer, R.; Stuart, W., Non-stoicheiometric clathrate compounds of water. Proc. R. Soc. 1957, 243, (1233), 172-189.

92. Van der Waals, J.; Platteeuw, J., Clathrate solutions. Adv. Chem. Phys 1959, 2, (1), 1-57.

93. Anderson, B.; Tester, J.; Trout, B., Accurate Potentials for Argon Water and Methane Water Interactions via ab Initio Methods and Their Application to Clathrate Hydrates. J. Phys. Chem. B 2004, 108, (48), 18705-18715.

94. Cao, Z.; Tester, J.; Sparks, K.; Trout, B., Molecular Computations Using Robust Hydrocarbon Water Potentials for Predicting Gas Hydrate Phase Equilibria. J. Phys. Chem. B 2001, 105, (44), 10950-10960.

95. Holder, G.; Corbin, G.; Papadopoulos, K., Thermodynamic and molecular properties of gas hydrates from mixtures containing methane, argon, and krypton. Industrial \& Engineering Chemistry Fundamentals 1980, 19, (3), 282-286.

96. John, V.; Papadopoulos, K.; Holder, G., A generalized model for predicting equilibrium conditions for gas hydrates. AlChE J. 1985, 31, (2), 252-259.

97. Bazant, M.; Trout, B., A method to extract potentials from the temperature dependence of Langmuir constants for clathrate-hydrates. Physica A 2001, 300, (1-2), 139-173.

98. Klauda, J.; Sandler, S., Ab initio intermolecular potentials for gas hydrates and their predictions. J. Phys. Chem. B 2002, 106, (22), 5722-5732.

99. Velaga, S. C. Phase equilibrium and cage occupancy calculations of carbon dioxide hydrates using ab initio intermolecular potentials. M.S.Thesis, WEST VIRGINIA UNIVERSITY, Morgantown, 2009. 
100. Velaga, S. C.; Anderson, B. J., Carbon Dioxide Hydrates Phase Equilibrium and Cage Occupancy Calculations Using Ab Initio Intermolecular Potentials. J. Phys. Chem. B 2013, $118,(2), 577-589$.

101. Ravipati, S.; Punnathanam, S. N., Analysis of Parameter Values in the van der Waals and Platteeuw Theory for Methane Hydrates Using Monte Carlo Molecular Simulations. Ind. Eng. Chem. Res. 2012, 51, (27), 9419-9426.

102. Lennard-Jones, J.; Devonshire, A., Critical phenomena in gases. II. vapour pressures and boiling points. Proc. R. Soc. 1938, 165, (920), 1-11.

103. Holder, G.; Zetts, S.; Pradhan, N., Phase behavior in systems containing clathrate hydrates. Rev. Chem. Eng 1988, 5, (1), 1-70.

104. Pradhan, N. Prediction of Multi-phase Equilibria in Gas hydrates. M.S. Thesis, University of Pittsburgh, Pittsburgh, 1985.

105. Sassen, R.; Roberts, H.; Jung, W.; Lutken, C.; DeFreitas, D.; Sweet, S.; Guinasso Jr, N. In The Mississippi Canyon 118 gas hydrate site: A complex natural system, Offshore Technology Conference, 2006; 2006.

106. Solomon, S., Climate change 2007: the physical science basis: contribution of Working Group I to the Fourth Assessment Report of the Intergovernmental Panel on Climate Change. Cambridge Univ Pr: 2007.

107. MacDonald, I.; Guinasso, N.; Sassen, R.; Brooks, J.; Lee, L.; Scott, K., Gas hydrate that breaches the sea floor on the continental slope of the Gulf of Mexico. Geology 1994, 22, (8), 699-702.

108. Paull, C. K.; Normark, W. R.; Ussler III, W.; Caress, D. W.; Keaten, R., Association among active seafloor deformation, mound formation, and gas hydrate growth and accumulation 
within the seafloor of the Santa Monica Basin, offshore California. Marine geology 2008, 250, (3), 258-275.

109. Bigalke, N. K.; Rehder, G.; Gust, G., Methane hydrate dissolution rates in undersaturated seawater under controlled hydrodynamic forcing. Mar. Chem. 2009, 115, (3-4), 226-234.

110. Rosenbaum, E. J.; Niall, J.; Johnson, J. K.; Shaw, D. W.; Warzinski, R. P., Thermal conductivity of methane hydrate from experiment and molecular simulation. J. Phys. Chem. B 2007, 111, (46), 13194-13205.

111. English, N. J.; MacElroy, J., Structural and dynamical properties of methane clathrate hydrates. J. Comput. Chem. 2003, 24, (13), 1569-1581.

112. Vatamanu, J.; Kusalik, P. G., Heterogeneous crystal growth of methane hydrate on its sii [001] crystallographic face. J. Phys. Chem. B 2008, 112, (8), 2399-2404.

113. Myshakin, E. M.; Jiang, H.; Warzinski, R. P.; Jordan, K. D., Molecular Dynamics Simulations of Methane Hydrate Decomposition†. J. Phys. Chem. A 2009, 113, (10), 19131921.

114. English, N. J.; Johnson, J.; Taylor, C. E., Molecular-dynamics simulations of methane hydrate dissociation. J. Chem. Phys. 2005, 123, 244503.

115. Alavi, S.; Ripmeester, J. A., Nonequilibrium adiabatic molecular dynamics simulations of methane clathrate hydrate decomposition. J. Chem. Phys. 2010, 132, (14), 8.

116. Anderson, B. J.; Tester, J. W.; Borghi, G. P.; Trout, B. L., Properties of inhibitors of methane hydrate formation via molecular dynamics simulations. J. Am. Chem. Soc. 2005, $127,(50), 17852-17862$.

117. Abascal, J.; Sanz, E.; Fernández, R. G.; Vega, C., A potential model for the study of ices and amorphous water: TIP4P/Ice. The Journal of chemical physics 2005, 122, 234511. 
118. Vega, C.; Abascal, J.; Conde, M.; Aragones, J., What ice can teach us about water interactions: a critical comparison of the performance of different water models. Faraday Discuss. 2009, 141, 251-276.

119. Conde, M.; Vega, C., Determining the three-phase coexistence line in methane hydrates using computer simulations. J. Chem. Phys. 2010, 133, (6), 064507-064507-12.

120. Conde, M.; Vega, C., Note: A simple correlation to locate the three phase coexistence line in methane-hydrate simulations. J. Chem. Phys. 2013, 138, 056101-056102.

121. Jorgensen, W. L.; Madura, J. D.; Swenson, C. J., Optimized intermolecular potential functions for liquid hydrocarbons. J. Am. Chem. Soc. 1984, 106, (22), 6638-6646.

122. Tester, J.; Modell, M., Thermodynamics and its Applications. Prentice Hall PTR Upper Saddle River, NJ: 1997.

123. Ewald, P., Evaluation of optical and electrostatic lattice potentials. Ann. Phys 1921, 64, 253.

124. Abascal, J.; Sanz, E.; Fernández, R. G.; Vega, C., A potential model for the study of ices and amorphous water: TIP4P/Ice. J. Chem. Phys. 2005, 122, 234511-234520.

125. Bernal, J.; Fowler, R., A theory of water and ionic solution, with particular reference to hydrogen and hydroxyl ions. J. chem. Phys 1933, 1, (8), 515-548.

126. Garapati, N. Determination of mixed hydrate thermodynamics for reservoir modeling. M.S., West Virginia University, 2009.

127. Larson, M.; Garside, J., Solute clustering in supersaturated solutions. Chem. Eng. Sci. 1986, $41,(5), 1285-1289$.

128. van der Spoel, D.; Lindahl, E.; Hess, B.; Van Buuren, A.; Apol, E.; Meulenhoff, P.; Tieleman, D.; Sijbers, A.; Feenstra, K.; van Drunen, R. Gromacs User manual version 3.3; www.gromacs.org, 2008. 
129. Nosé, S., A molecular dynamics method for simulations in the canonical ensemble. Mol. Phys. 2002, 100, (1), 191-198.

130. Parrinello, M.; Rahman, A., Polymorphic transitions in single crystals: A new molecular dynamics method. J. Appl. Phys. 1981, 52, (12), 7182-7190.

131. Ciccotti, G.; Ryckaert, J. P., Molecular dynamics simulation of rigid molecules. Comput. Phys. Rep. 1986, 4, (6), 346-392.

132. Vedam, V. S. Stability of carbon dioxide and methane hydrates in water in presence of small driving forces using MD simulations. M.S. Thesis, West Virginia University, Morgantown, 2010.

133. Velaga, S.; Vedam, V.; Anderson, B. J. In Molecular dynamics studies of the stability of $\mathrm{CO}_{2}$ and $\mathrm{CH}_{4}$ hydrates in the presence of undersaturated fluids, 7th International Conference on Gas Hydrates (ICGH ), Edinburgh, 17-21 July 2011; Edinburgh, 2011.

134. Sloan, E. D.; Koh, C. A., Clathrate hydrates of natural gases. CRC: 2008.

135. Zele, S.; Lee, S.; Holder, G., A theory of lattice distortion in gas hydrates. J. Phys. Chem. B 1999, 103, (46), 10250-10257.

136. Lee, S. Y.; Holder, G. D., Model for gas hydrate equilibria using a variable reference chemical potential: Part 1. AlChE J. 2002, 48, (1), 161-167.

137. Garapati, N.; Anderson, B. J., Predictions of Mixed Hydrate Phase Equilibria and the Swapping of $\mathrm{CH} 4$ Hydrate with $\mathrm{CO} 2$ and $\mathrm{CO} 2+\mathrm{N} 2$ Mixtures.

138. Garapati, N., Statistical Thermodynamics Model and Empirical Correlations for Predicting Mixed Hydrate Phase Equilibria. Fluid Phase Equilib. In review. 
139. Velaga, S. Phase equilibrium and cage occupancy calculations of carbon dioxide hydrates using ab initio intermolecular potentials. M.S. Thesis, WEST VIRGINIA UNIVERSITY, 2009.

140. Peng, D.; Robinson, D., A New Two-Constant Equation of State. Ind. Eng. Chem. Fundam. 1976, 15, 59-64

141. Carroll, J. J.; Mather, A. E., A model for the solubility of light hydrocarbons in water and aqueous solutions of alkanolamines. Chem. Eng. Sci. 1997, 52, (4), 545-552.

142. Peng, D.; Robinson, D., A new cubic equation of state. Ind. Eng. Chem. Fund 1976, 15, 59.

143. Yaws, C. L.; Hopper, J. R.; XIAOMEI, W.; Rathinsamy, A. K.; Pike, R. W., Calculating solubility \& Henry's law constants for gases in water. Chemical engineering 1999, 106, (6), 102-105.

144. Chapoy, A.; Mokraoui, S.; Valtz, A.; Richon, D.; Mohammadi, A. H.; Tohidi, B., Solubility measurement and modeling for the system propane-water from 277.62 to 368.16 K. Fluid Phase Equilib. 2004, 226, 213-220.

145. Culberson, O.; McKetta Jr, J., Phase equilibria in hydrocarbon-water systems III-the solubility of methane in water at pressures to 10,000 psia. Journal of Petroleum Technology 1951, 3, (8), 223-226.

146. Kobayashi, R.; Katz, D., Vapor-liquid equilibria for binary hydrocarbon-water systems. Ind. Eng. Chem. 1953, 45, (2), 440-446.

147. Pitzer, K. S., Activity coefficients in electrolyte solutions. CRC press: Boston, 1991.

148. Spencer, R. J.; Møller, N.; Weare, J. H., The prediction of mineral solubilities in natural waters: A chemical equilibrium model for the $\mathrm{Na}^{+} \mathrm{K}^{+} \mathrm{Ca}^{2+} \mathrm{Mg}^{2+} \mathrm{Cl}^{-} \mathrm{SO}_{4}{ }^{2-} \mathrm{H}_{2} \mathrm{O}$ system at temperatures below $25^{\circ}$ C. Geochim. Cosmochim. Acta 1990, 54, (3), 575-590. 
149. Monnin, C., The influence of pressure on the activity coefficients of the solutes and on the solubility of minerals in the system Na-Ca-Cl-SO $4</$ sub $>-\mathrm{H}<\mathrm{sub}>2</$ sub $>0$ to $200^{\circ} \mathrm{C}$ and $1 \mathrm{kbar}$ and to high $\mathrm{NaCl}$ concentration. Geochim. Cosmochim. Acta 1990, 54, (12), 32653282.

150. Millero, F., The activity coefficients of non-electrolytes in seawater. Mar. Chem. 2000, 70, (1), 5-22.

151. Mohammadi, A. H.; Richon, D., A mathematical model based on artificial neural network technique for estimating liquid water- hydrate equilibrium of water- hydrocarbon System. Industrial \& Engineering Chemistry Research 2008, 47, (14), 4966-4970.

152. Chou, I. M.; Burruss, R. C., Personal Communication 2006.

153. Huo, Z.; Hester, K.; Sloan, E. D.; Miller, K. T., Methane hydrate nonstoichiometry and phase diagram. AlChE J. 2003, 49, (5), 1300-1306.

154. Kneafsey, T. J.; Tomutsa, L.; Moridis, G. J.; Seol, Y.; Freifeld, B. M.; Taylor, C. E.; Gupta, A., Methane hydrate formation and dissociation in a partially saturated core-scale sand sample. Journal of Petroleum Science and Engineering 2007, 56, (1-3), 108-126.

155. Yang, S. O. Measurements and Predictions of Phase Equilibria for Water + Natural Gas Components in Hydrate Forming Conditions. PhD, Korea University, 2000.

156. Millero, F. J., Chemical Oceanography. 2 nd ed ed.; CRC press, Inc.: Boca Raton, FL, 1996. 


\section{Appendix A}

The oxygen and hydrogen positions of water in sII hydrate unit cell

\begin{tabular}{|c|c|c|c|c|c|c|c|c|c|}
\hline & \multicolumn{3}{|c|}{0} & \multicolumn{3}{|c|}{ H1 } & \multicolumn{3}{|c|}{ H2 } \\
\hline & $x$ & $y$ & z & $x$ & $y$ & z & $x$ & $y$ & z \\
\hline 1 & 0.1250 & 0.1250 & 0.1250 & 0.0941 & 0.0912 & 0.0941 & 0.1559 & 0.0912 & 0.1559 \\
\hline 2 & 0.8750 & 0.3750 & 0.8750 & 0.9059 & 0.4088 & 0.9059 & 0.8441 & 0.4088 & 0.8441 \\
\hline 3 & 0.0327 & 0.0327 & 0.0327 & 0.0458 & 0.0416 & -0.0203 & 0.0458 & -0.0203 & 0.0416 \\
\hline 4 & 0.2173 & 0.7173 & 0.5327 & 0.2085 & 0.7042 & 0.4797 & 0.2703 & 0.7042 & 0.5416 \\
\hline 5 & 0.7173 & 0.5327 & 0.2173 & 0.7044 & 0.5456 & 0.2695 & 0.6848 & 0.5652 & 0.1865 \\
\hline 6 & 0.5327 & 0.2173 & 0.7173 & 0.5416 & 0.2042 & 0.7703 & 0.4797 & 0.2042 & 0.7085 \\
\hline 7 & 0.7827 & 0.2827 & 0.9673 & 0.8152 & 0.3152 & 0.9365 & 0.7956 & 0.2956 & 1.0195 \\
\hline 8 & 0.4673 & 0.4673 & 0.4673 & 0.4585 & 0.5203 & 0.4542 & 0.5203 & 0.4585 & 0.4542 \\
\hline 9 & 0.2827 & 0.9673 & 0.7827 & 0.2297 & 0.9585 & 0.7958 & 0.2916 & 1.0203 & 0.7958 \\
\hline 10 & 0.9673 & 0.7827 & 0.2827 & 0.9365 & 0.8152 & 0.3152 & 1.0195 & 0.7956 & 0.2956 \\
\hline 11 & 0.0676 & 0.0676 & 0.8801 & 0.1054 & 0.1079 & 0.8793 & 0.0223 & 0.0907 & 0.8585 \\
\hline 12 & 0.1824 & 0.6824 & 0.3801 & 0.1446 & 0.6421 & 0.3793 & 0.2278 & 0.6593 & 0.3585 \\
\hline 13 & 0.6824 & 0.5676 & 0.3699 & 0.6667 & 0.5215 & 0.3960 & 0.7285 & 0.5833 & 0.3960 \\
\hline 14 & 0.5676 & 0.1824 & 0.8699 & 0.6054 & 0.1421 & 0.8707 & 0.5223 & 0.1593 & 0.8915 \\
\hline 15 & 0.8801 & 0.0676 & 0.0676 & 0.8592 & 0.0211 & 0.0889 & 0.9326 & 0.0550 & 0.0558 \\
\hline 16 & 0.3801 & 0.1824 & 0.6824 & 0.3540 & 0.1667 & 0.7285 & 0.3540 & 0.2285 & 0.6667 \\
\hline 17 & 0.3699 & 0.6824 & 0.5676 & 0.3960 & 0.6667 & 0.5215 & 0.3960 & 0.7285 & 0.5833 \\
\hline 18 & 0.8699 & 0.5676 & 0.1824 & 0.8174 & 0.5558 & 0.1950 & 0.8908 & 0.5889 & 0.2289 \\
\hline 19 & 0.0676 & 0.8801 & 0.0676 & 0.0833 & 0.8540 & 0.0215 & 0.0215 & 0.8540 & 0.0833 \\
\hline 20 & 0.6824 & 0.3801 & 0.1824 & 0.6942 & 0.4326 & 0.1950 & 0.6611 & 0.3592 & 0.2289 \\
\hline 21 & 0.5676 & 0.3699 & 0.6824 & 0.5558 & 0.3174 & 0.6950 & 0.5889 & 0.3908 & 0.7289 \\
\hline 22 & 0.1824 & 0.8699 & 0.5676 & 0.1933 & 0.8168 & 0.5567 & 0.1433 & 0.8683 & 0.6067 \\
\hline 23 & 0.8176 & 0.3176 & 0.1199 & 0.7723 & 0.3407 & 0.1415 & 0.8554 & 0.3579 & 0.1207 \\
\hline 24 & 0.4324 & 0.4324 & 0.6199 & 0.4450 & 0.4442 & 0.5674 & 0.4789 & 0.4111 & 0.6408 \\
\hline 25 & 0.3176 & 0.9324 & 0.6301 & 0.3050 & 0.9442 & 0.6826 & 0.2711 & 0.9111 & 0.6092 \\
\hline 26 & 0.9324 & 0.8176 & 0.1301 & 0.9442 & 0.8050 & 0.1826 & 0.9111 & 0.7711 & 0.1092 \\
\hline 27 & 0.8176 & 0.1301 & 0.9324 & 0.8058 & 0.1826 & 0.9450 & 0.8389 & 0.1092 & 0.9789 \\
\hline 28 & 0.9324 & 0.6301 & 0.3176 & 0.9433 & 0.6832 & 0.3067 & 0.8933 & 0.6317 & 0.3567 \\
\hline 29 & 0.4324 & 0.6199 & 0.4324 & 0.4778 & 0.6415 & 0.4093 & 0.3946 & 0.6207 & 0.3921 \\
\hline 30 & 0.3176 & 0.1199 & 0.8176 & 0.2723 & 0.1415 & 0.8407 & 0.3554 & 0.1207 & 0.8579 \\
\hline 31 & 0.6301 & 0.3176 & 0.9324 & 0.6826 & 0.3050 & 0.9442 & 0.6092 & 0.2711 & 0.9111 \\
\hline 32 & 0.1301 & 0.9324 & 0.8176 & 0.1293 & 0.8946 & 0.8579 & 0.1085 & 0.9778 & 0.8407 \\
\hline 33 & 0.1199 & 0.8176 & 0.3176 & 0.1460 & 0.7715 & 0.3333 & 0.1460 & 0.8333 & 0.2715 \\
\hline 34 & 0.6199 & 0.4324 & 0.4324 & 0.6415 & 0.4093 & 0.4778 & 0.6207 & 0.3921 & 0.3946 \\
\hline 35 & 0.1250 & 0.6250 & 0.6250 & 0.1588 & 0.6559 & 0.5941 & 0.1588 & 0.5941 & 0.6559 \\
\hline 36 & 0.8750 & 0.8750 & 0.3750 & 0.8441 & 0.8441 & 0.4088 & 0.9059 & 0.9059 & 0.4088 \\
\hline 37 & 0.0327 & 0.5327 & 0.5327 & 0.0652 & 0.5652 & 0.5635 & 0.0456 & 0.5456 & 0.4805 \\
\hline 38 & 0.2173 & 0.2173 & 0.0327 & 0.1848 & 0.1865 & 0.0652 & 0.2044 & 0.2695 & 0.0456 \\
\hline 39 & 0.7173 & 0.0327 & 0.7173 & 0.7042 & 0.0416 & 0.7703 & 0.7042 & -0.0203 & 0.7085 \\
\hline 40 & 0.5327 & 0.7173 & 0.2173 & 0.5456 & 0.7695 & 0.2044 & 0.5652 & 0.6865 & 0.1848 \\
\hline 41 & 0.7827 & 0.7827 & 0.4673 & 0.7916 & 0.7958 & 0.5203 & 0.7297 & 0.7958 & 0.4585 \\
\hline 42 & 0.4673 & 0.9673 & 0.9673 & 0.5195 & 0.9544 & 0.9544 & 0.4365 & 0.9348 & 0.9348 \\
\hline 43 & 0.2827 & 0.4673 & 0.2827 & 0.2916 & 0.4542 & 0.2297 & 0.2297 & 0.4542 & 0.2916 \\
\hline
\end{tabular}




\begin{tabular}{|c|c|c|c|c|c|c|c|c|c|}
\hline 44 & 0.9673 & 0.2827 & 0.7827 & 0.9348 & 0.3135 & 0.8152 & 0.9544 & 0.2305 & 0.7956 \\
\hline 45 & 0.0676 & 0.5676 & 0.3801 & 0.0833 & 0.5215 & 0.3540 & 0.0215 & 0.5833 & 0.3540 \\
\hline 46 & 0.1824 & 0.1824 & 0.8801 & 0.1611 & 0.2289 & 0.8592 & 0.1942 & 0.1950 & 0.9326 \\
\hline 47 & 0.6824 & 0.0676 & 0.8699 & 0.7285 & 0.0833 & 0.8960 & 0.6667 & 0.0215 & 0.8960 \\
\hline 48 & 0.5676 & 0.6824 & 0.3699 & 0.6067 & 0.6433 & 0.3683 & 0.5567 & 0.6933 & 0.3168 \\
\hline 49 & 0.8801 & 0.5676 & 0.5676 & 0.8592 & 0.5211 & 0.5889 & 0.9326 & 0.5550 & 0.5558 \\
\hline 50 & 0.3801 & 0.6824 & 0.1824 & 0.4332 & 0.6933 & 0.1933 & 0.3817 & 0.6433 & 0.1433 \\
\hline 51 & 0.3699 & 0.1824 & 0.0676 & 0.3174 & 0.1950 & 0.0558 & 0.3908 & 0.2289 & 0.0889 \\
\hline 52 & 0.8699 & 0.0676 & 0.6824 & 0.8168 & 0.0567 & 0.6933 & 0.8683 & 0.1067 & 0.6433 \\
\hline 53 & 0.0676 & 0.3801 & 0.5676 & 0.0567 & 0.4332 & 0.5567 & 0.1067 & 0.3817 & 0.6067 \\
\hline 54 & 0.6824 & 0.8801 & 0.6824 & 0.7278 & 0.8585 & 0.6593 & 0.6446 & 0.8793 & 0.6421 \\
\hline 55 & 0.5676 & 0.8699 & 0.1824 & 0.5215 & 0.8960 & 0.1667 & 0.5833 & 0.8960 & 0.2285 \\
\hline 56 & 0.1824 & 0.3699 & 0.0676 & 0.1421 & 0.3707 & 0.1054 & 0.1593 & 0.3915 & 0.0223 \\
\hline 57 & 0.8176 & 0.8176 & 0.6199 & 0.8407 & 0.7723 & 0.6415 & 0.8579 & 0.8554 & 0.6207 \\
\hline 58 & 0.4324 & 0.9324 & 0.1199 & 0.3933 & 0.8933 & 0.1183 & 0.4433 & 0.9433 & 0.0668 \\
\hline 59 & 0.3176 & 0.4324 & 0.1301 & 0.2723 & 0.4093 & 0.1085 & 0.3554 & 0.3921 & 0.1293 \\
\hline 60 & 0.9324 & 0.3176 & 0.6301 & 0.9450 & 0.3058 & 0.6826 & 0.9789 & 0.3389 & 0.6092 \\
\hline 61 & 0.8176 & 0.6301 & 0.4324 & 0.8058 & 0.6826 & 0.4450 & 0.8389 & 0.6092 & 0.4789 \\
\hline 62 & 0.9324 & 0.1301 & 0.8176 & 0.8921 & 0.1293 & 0.8554 & 0.9093 & 0.1085 & 0.7723 \\
\hline 63 & 0.4324 & 0.1199 & 0.9324 & 0.4442 & 0.0674 & 0.9450 & 0.4111 & 0.1408 & 0.9789 \\
\hline 64 & 0.3176 & 0.6199 & 0.3176 & 0.3058 & 0.5674 & 0.3050 & 0.3389 & 0.6408 & 0.2711 \\
\hline 65 & 0.6301 & 0.8176 & 0.4324 & 0.6085 & 0.7723 & 0.4093 & 0.6293 & 0.8554 & 0.3921 \\
\hline 66 & 0.1301 & 0.4324 & 0.3176 & 0.1085 & 0.4093 & 0.2723 & 0.1293 & 0.3921 & 0.3554 \\
\hline 67 & 0.1199 & 0.3176 & 0.8176 & 0.1408 & 0.3389 & 0.7711 & 0.0674 & 0.3058 & 0.8050 \\
\hline 68 & 0.6199 & 0.9324 & 0.9324 & 0.6207 & 0.8921 & 0.8946 & 0.6415 & 0.9093 & 0.9778 \\
\hline 69 & 0.6250 & 0.1250 & 0.6250 & 0.5941 & 0.1559 & 0.6588 & 0.6559 & 0.0941 & 0.6588 \\
\hline 70 & 0.3750 & 0.3750 & 0.3750 & 0.4059 & 0.4088 & 0.4059 & 0.3441 & 0.4088 & 0.3441 \\
\hline 71 & 0.5327 & 0.0327 & 0.5327 & 0.5635 & 0.0652 & 0.5652 & 0.4805 & 0.0456 & 0.5456 \\
\hline 72 & 0.7173 & 0.7173 & 0.0327 & 0.7042 & 0.7703 & 0.0416 & 0.7042 & 0.7085 & -0.0203 \\
\hline 73 & 0.2173 & 0.5327 & 0.7173 & 0.2085 & 0.5458 & 0.7703 & 0.2703 & 0.5458 & 0.7085 \\
\hline 74 & 0.0327 & 0.2173 & 0.2173 & 0.0635 & 0.1848 & 0.1848 & -0.0195 & 0.2044 & 0.2044 \\
\hline 75 & 0.2827 & 0.2827 & 0.4673 & 0.3152 & 0.3135 & 0.4348 & 0.2956 & 0.2305 & 0.4544 \\
\hline 76 & 0.9673 & 0.4673 & 0.9673 & 0.9585 & 0.5203 & 0.9542 & 1.0203 & 0.4585 & 0.9542 \\
\hline 77 & 0.7827 & 0.9673 & 0.2827 & 0.8152 & 0.9365 & 0.3152 & 0.7956 & 1.0195 & 0.2956 \\
\hline 78 & 0.4673 & 0.7827 & 0.7827 & 0.4542 & 0.7916 & 0.7297 & 0.4542 & 0.7297 & 0.7916 \\
\hline 79 & 0.5676 & 0.0676 & 0.3801 & 0.5550 & 0.0558 & 0.4326 & 0.5211 & 0.0889 & 0.3592 \\
\hline 80 & 0.6824 & 0.6824 & 0.8801 & 0.6667 & 0.7285 & 0.8540 & 0.7285 & 0.6667 & 0.8540 \\
\hline 81 & 0.1824 & 0.5676 & 0.8699 & 0.1446 & 0.6079 & 0.8707 & 0.2278 & 0.5907 & 0.8915 \\
\hline 82 & 0.0676 & 0.1824 & 0.3699 & 0.0558 & 0.1950 & 0.3174 & 0.0889 & 0.2289 & 0.3908 \\
\hline 83 & 0.3801 & 0.0676 & 0.5676 & 0.3793 & 0.1054 & 0.6079 & 0.3585 & 0.0223 & 0.5907 \\
\hline 84 & 0.8801 & 0.1824 & 0.1824 & 0.8793 & 0.1446 & 0.1421 & 0.8585 & 0.2278 & 0.1593 \\
\hline 85 & 0.8699 & 0.6824 & 0.0676 & 0.8683 & 0.6433 & 0.1067 & 0.8168 & 0.6933 & 0.0567 \\
\hline 86 & 0.3699 & 0.5676 & 0.6824 & 0.3707 & 0.6054 & 0.6421 & 0.3915 & 0.5223 & 0.6593 \\
\hline 87 & 0.5676 & 0.8801 & 0.5676 & 0.5889 & 0.8592 & 0.5211 & 0.5558 & 0.9326 & 0.5550 \\
\hline 88 & 0.1824 & 0.3801 & 0.6824 & 0.1950 & 0.4326 & 0.6942 & 0.2289 & 0.3592 & 0.6611 \\
\hline 89 & 0.0676 & 0.3699 & 0.1824 & 0.0550 & 0.3174 & 0.1942 & 0.0211 & 0.3908 & 0.1611 \\
\hline 90 & 0.6824 & 0.8699 & 0.0676 & 0.6446 & 0.8707 & 0.1079 & 0.7278 & 0.8915 & 0.0907 \\
\hline 91 & 0.3176 & 0.3176 & 0.6199 & 0.3567 & 0.3567 & 0.6183 & 0.3067 & 0.3067 & 0.5668 \\
\hline 92 & 0.9324 & 0.4324 & 0.1199 & 0.9111 & 0.4789 & 0.1408 & 0.9442 & 0.4450 & 0.0674 \\
\hline 93 & 0.8176 & 0.9324 & 0.1301 & 0.8567 & 0.8933 & 0.1317 & 0.8067 & 0.9433 & 0.1832 \\
\hline
\end{tabular}




\begin{tabular}{|c|c|c|c|c|c|c|c|c|c|}
\hline 94 & 0.4324 & 0.8176 & 0.6301 & 0.3946 & 0.8579 & 0.6293 & 0.4778 & 0.8407 & 0.6085 \\
\hline 95 & 0.3176 & 0.1301 & 0.4324 & 0.3333 & 0.1040 & 0.4785 & 0.2715 & 0.1040 & 0.4167 \\
\hline 96 & 0.4324 & 0.6301 & 0.8176 & 0.4093 & 0.6085 & 0.7723 & 0.3921 & 0.6293 & 0.8554 \\
\hline 97 & 0.9324 & 0.6199 & 0.9324 & 0.8921 & 0.6207 & 0.8946 & 0.9093 & 0.6415 & 0.9778 \\
\hline 98 & 0.8176 & 0.1199 & 0.3176 & 0.8407 & 0.1415 & 0.2723 & 0.8579 & 0.1207 & 0.3554 \\
\hline 99 & 0.1301 & 0.3176 & 0.4324 & 0.1092 & 0.3389 & 0.4789 & 0.1826 & 0.3058 & 0.4450 \\
\hline 100 & 0.6301 & 0.9324 & 0.3176 & 0.6826 & 0.9450 & 0.3058 & 0.6092 & 0.9789 & 0.3389 \\
\hline 101 & 0.6199 & 0.8176 & 0.8176 & 0.6408 & 0.8389 & 0.7711 & 0.5674 & 0.8058 & 0.8050 \\
\hline 102 & 0.1199 & 0.4324 & 0.9324 & 0.1207 & 0.3946 & 0.8921 & 0.1415 & 0.4778 & 0.9093 \\
\hline 103 & 0.6250 & 0.6250 & 0.1250 & 0.6559 & 0.6559 & 0.0912 & 0.5941 & 0.5941 & 0.0912 \\
\hline 104 & 0.3750 & 0.8750 & 0.8750 & 0.3441 & 0.9059 & 0.8412 & 0.4059 & 0.8441 & 0.8412 \\
\hline 105 & 0.5327 & 0.5327 & 0.0327 & 0.5458 & 0.4797 & 0.0416 & 0.5458 & 0.5416 & -0.0203 \\
\hline 106 & 0.7173 & 0.2173 & 0.5327 & 0.6848 & 0.1848 & 0.5635 & 0.7044 & 0.2044 & 0.4805 \\
\hline 107 & 0.2173 & 0.0327 & 0.2173 & 0.2085 & 0.0458 & 0.2703 & 0.2703 & 0.0458 & 0.2085 \\
\hline 108 & 0.0327 & 0.7173 & 0.7173 & 0.0652 & 0.6865 & 0.6848 & 0.0456 & 0.7695 & 0.7044 \\
\hline 109 & 0.2827 & 0.7827 & 0.9673 & 0.3152 & 0.8152 & 0.9365 & 0.2956 & 0.7956 & 1.0195 \\
\hline 110 & 0.9673 & 0.9673 & 0.4673 & 1.0203 & 0.9585 & 0.4542 & 0.9585 & 1.0203 & 0.4542 \\
\hline 111 & 0.7827 & 0.4673 & 0.7827 & 0.7916 & 0.4542 & 0.7297 & 0.7297 & 0.4542 & 0.7916 \\
\hline 112 & 0.4673 & 0.2827 & 0.2827 & 0.4348 & 0.3135 & 0.3152 & 0.4544 & 0.2305 & 0.2956 \\
\hline 113 & 0.5676 & 0.5676 & 0.8801 & 0.6054 & 0.6079 & 0.8793 & 0.5223 & 0.5907 & 0.8585 \\
\hline 114 & 0.6824 & 0.1824 & 0.3801 & 0.6446 & 0.1421 & 0.3793 & 0.7278 & 0.1593 & 0.3585 \\
\hline 115 & 0.1824 & 0.0676 & 0.3699 & 0.1421 & 0.1054 & 0.3707 & 0.1593 & 0.0223 & 0.3915 \\
\hline 116 & 0.0676 & 0.6824 & 0.8699 & 0.0550 & 0.6942 & 0.8174 & 0.0211 & & 0.8908 \\
\hline 117 & 0.3801 & 0.5676 & 0.0676 & 0.3592 & 0.5211 & 0.0889 & 0.4326 & 550 & 0.0558 \\
\hline 118 & 0.8801 & 0.6824 & 0.6824 & 0.8817 & 0.6433 & 0.6433 & 0.9332 & 0.6933 & 0.6933 \\
\hline 119 & 0.8699 & 0.1824 & 0.5676 & 0.8908 & 0.2289 & 0.5889 & 0.8174 & 0.1950 & 0.5558 \\
\hline 120 & 0.3699 & 0.0676 & 0.1824 & 0.3707 & 0.1054 & 0.1421 & 0.3915 & 0.0223 & 0.1593 \\
\hline 121 & 0.5676 & 0.3801 & 0.0676 & 0.6079 & 0.3793 & 0.1054 & 0.5907 & 0.3585 & 0.0223 \\
\hline 122 & 0.1824 & 0.8801 & 0.1824 & 0.1433 & 0.8817 & 0.1433 & 0.1933 & 0.9332 & 0.1933 \\
\hline 123 & 0.0676 & 0.8699 & 0.6824 & 0.0833 & & & & & 0.6667 \\
\hline 124 & 0.6824 & 0.3699 & 0.5676 & 0.6433 & 0.3683 & 0.6067 & 0.6933 & 0.3168 & 0.5567 \\
\hline 125 & 0.3176 & 0.8176 & 0.1199 & 0.3333 & 0.7715 & 0.1460 & 0.2715 & 0.8333 & 0.1460 \\
\hline 126 & 0.9324 & 0.9324 & 0.6199 & 0.9442 & 0.9450 & 0.5674 & 0.9111 & 0.9789 & 0.6408 \\
\hline 127 & 0.8176 & 0.4324 & 0.6301 & 0.8554 & 0.3921 & 0.6293 & 0.7723 & 0.4093 & 0.6085 \\
\hline 128 & 0.4324 & 0.3176 & 0.1301 & 0.4450 & 0.3058 & 0.1826 & 0.4789 & 0.3389 & 0.1092 \\
\hline 129 & 0.3176 & 0.6301 & 0.9324 & 0.3058 & 0.6826 & & & & 0.9789 \\
\hline 130 & 0.4324 & 0.1301 & 0.3176 & 0.3921 & 0.1293 & 0.3554 & 0.4093 & 0.1085 & 0.2723 \\
\hline 131 & 0.9324 & 0.1199 & 0.4324 & 0.9167 & 0.1460 & 0.4785 & 0.9785 & 0.1460 & 0.4167 \\
\hline 132 & 0.8176 & 0.6199 & 0.8176 & 0.8058 & 0.5674 & 0.8050 & 0.8389 & 0.6408 & 0.7711 \\
\hline 133 & 0.1301 & 0.8176 & 0.9324 & 0.1826 & 0.8050 & 0.9442 & 0.1092 & 0.7711 & 0.9111 \\
\hline 134 & 0.6301 & 0.4324 & 0.8176 & 0.6293 & 0.3946 & 0.8579 & 0.6085 & 0.4778 & 0.8407 \\
\hline 135 & 0.6199 & 0.3176 & 0.3176 & 0.5674 & 0.3050 & 0.3058 & 0.6408 & 0.2711 & 0.3389 \\
\hline 136 & 0.1199 & 0.9324 & 0.4324 & 0.1415 & 0.9093 & 0.4778 & 0.1207 & 0.8921 & 0.3946 \\
\hline
\end{tabular}

